\title{
Leptonic Pion Decay And Physics Beyond The Electroweak Standard Model
}

by

\author{
Ahmed Ismail \\ A Thesis submitted to \\ the Faculty of Graduate Studies and Research \\ in partial fulfilment of \\ the requirements for the degree of \\ Master of Science
}

Ottawa-Carleton Institute for Physics

\author{
Department of Physics \\ Carleton University \\ Ottawa, Ontario, Canada \\ September 2008
}

Copyright (C)

2008 - Ahmed Ismail 


$\begin{array}{ll}\begin{array}{l}\text { Library and } \\ \text { Archives Canada }\end{array} & \begin{array}{l}\text { Bibliothèque et } \\ \text { Archives Canada }\end{array} \\ \begin{array}{l}\text { Published Heritage } \\ \text { Branch }\end{array} & \begin{array}{l}\text { Direction du } \\ \text { Patrimoine de l'édition }\end{array} \\ \begin{array}{l}\text { 395 Wellington Street } \\ \text { Ottawa ON K1A 0N4 } \\ \text { Canada }\end{array} & \begin{array}{l}\text { 395, rue Wellington } \\ \text { Ottawa ON K1A 0N4 } \\ \text { Canada }\end{array}\end{array}$

Your file Votre référence ISBN: 978-0-494-44126-8 Ourfile Notre référence ISBN: 978-0-494-44126-8

NOTICE:

The author has granted a nonexclusive license allowing Library and Archives Canada to reproduce, publish, archive, preserve, conserve, communicate to the public by telecommunication or on the Internet, loan, distribute and sell theses worldwide, for commercial or noncommercial purposes, in microform, paper, electronic and/or any other formats.

The author retains copyright ownership and moral rights in this thesis. Neither the thesis nor substantial extracts from it may be printed or otherwise reproduced without the author's permission.
AVIS:

L'auteur a accordé une licence non exclusive permettant à la Bibliothèque et Archives Canada de reproduire, publier, archiver, sauvegarder, conserver, transmettre au public par télécommunication ou par l'Internet, prêter, distribuer et vendre des thèses partout dans le monde, à des fins commerciales ou autres, sur support microforme, papier, électronique et/ou autres formats.

L'auteur conserve la propriété du droit d'auteur et des droits moraux qui protège cette thèse. $\mathrm{Ni}$ la thèse ni des extraits substantiels de celle-ci ne doivent être imprimés ou autrement reproduits sans son autorisation.
In compliance with the Canadian Privacy Act some supporting forms may have been removed from this thesis.

While these forms may be included in the document page count, their removal does not represent any loss of content from the thesis.
Conformément à la loi canadienne sur la protection de la vie privée, quelques formulaires secondaires ont été enlevés de cette thèse.

Bien que ces formulaires aient inclus dans la pagination, il n'y aura aucun contenu manquant.

\section{Canada}




\section{Abstract}

In the Standard Model of particle physics the decay $\pi \rightarrow l \nu$ is chirally suppressed due to the $V-A$ structure of the weak interactions, predicting $\frac{\Gamma(\pi \rightarrow e \nu)}{\Gamma(\pi \rightarrow \mu \nu)}=$ $(1.2352 \pm 0.0001) \cdot 10^{-4}$, in agreement with precision measurements of this ratio. New fundamental interactions may contribute to this decay, potentially upsetting the precise agreement between the theoretically predicted, and the experimentally observed, ratio of branching ratios. New pseudoscalar interactions would contribute to the decay amplitude without chiral suppression, so it is highly sensitive to their presence; these pseudoscalars may themselves be induced by weak-interaction dressing of new scalar interactions. We analyze constraints on new axial-vector, pseudoscalar, and scalar interactions, arising in several proposed extensions of the Standard Model, from their effects on the ratio of branching ratios for $\pi \rightarrow l \nu$ decay, including one-loop QCD corrections to such amplitudes which we calculate. 


\section{Acknowledgements}

I would like to thank my supervisor, Bruce Campbell, for his guidance and unwavering support throughout my B.Sc. and M.Sc. studies. Bruce has taught me an incredible amount of physics over the past four years, and I particularly appreciate his assistance in the development of this thesis. It has been my privilege to work with him.

I have also benefited greatly from discussions with Heather Logan and David Maybury, as well as many other members of the Carleton University Department of Physics. It is my pleasure to thank the Department for providing financial aid during my studies, as well as a friendly and supportive environment.

Finally, I would like to thank my family for encouraging me throughout my education. This work was supported by the Natural Sciences and Engineering Research Council of Canada. 


\section{Table of Contents}

Abstract

Acknowledgements $\quad$ iii

Table of Contents $\quad$ iv

List of Tables vii

List of Figures viii

1 The Standard Model $\quad 1$

1.1 Introduction . . . . . . . . . . . . . . . . 1

1.2 Gauge Theory . . . . . . . . . . . . . . . . 3

1.2.1 Euler-Lagrange Equations of Motion . . . . . . . . . . 3

1.2.2 Noether's Theorem ................. . . 4

1.2.3 Gauging Symmetries . . . . . . . . . . . . . . 6

1.2.4 Non-Abelian Gauge Groups ... . . . . . . . . . 8

1.3 Spontaneous Symmetry Breaking . . . . . . . . . . . . . 14

1.3.1 $\phi^{4}$ Theory ................................ 15

1.3.2 Electroweak theory . . . . . . . . . . . . 16

1.4 The Standard Model Lagrangian . . . . . . . . . . . . 25

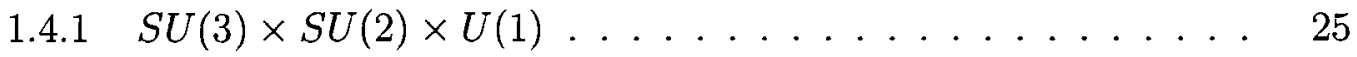


1.4.2 Calculating Amplitudes _. . . . . . . . . . . 27

List of References . . . . . . . . . . . . . . . . . . 30

2 Effective Field Theory 33

2.1 Renormalization . . . . . . . . . . . . . . . . 33

2.1 .1 Dimensional Regularization $\ldots \ldots \ldots \ldots \ldots$

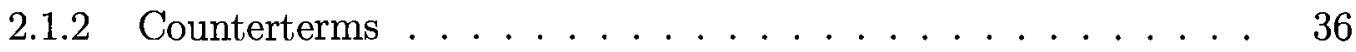

2.1.3 The Hierarchy Problem . . . . . . . . . . . . . . 38

2.1.4 The Callan-Symanzik Equation . . . . . . . . . . . . 39

2.1.5 Local Operators . . . . . . . . . . . . . . . . . . 43

2.2 Example: Fermi Theory Of Weak Interactions . . . . . . . . . 46

2.3 Example: Effective Field Theory Of $\Delta S=2$ Interactions In The Standard Model . . . . . . . . . . . . . . . . . . 47

2.3 .1 The GIM Mechanism . . . . . . . . . . . . 48

2.3.2 Integrating Out $t \ldots \ldots \ldots \ldots \ldots \ldots$

2.3.3 Renormalization of The $\Delta S=2$ Operator $\ldots \ldots \ldots \ldots 2$

2.3 .4 Integrating Out $c \ldots \ldots \ldots \ldots \ldots$

2.3 .5 QCD Corrections . . . . . . . . . . . . . 54

List of References . . . . . . . . . . . . . . 56

3 Leptonic Pion Decay And Physics Beyond The Standard Model 57

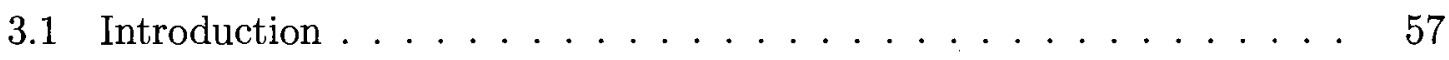

3.2 Effect of Pseudoscalar Interaction $\ldots \ldots \ldots \ldots \ldots$

3.3 Limits $\ldots \ldots \ldots \ldots \ldots \ldots$. . . . . . . . . . . . . . . 61

3.3.1 R-Parity Violating Supersymmetry . . . . . . . . . . . 61

3.3 .2 Leptoquarks . . . . . . . . . . . . . . . . 6 67

3.3.3 Extra Higgs Bosons . . . . . . . . . . . . . . . . . . 71

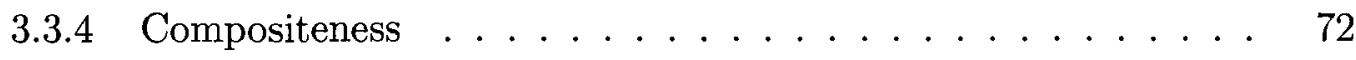


3.3.5 Supercompositeness . . . . . . . . . . . . . 76

List of References . . . . . . . . . . . . . . . . . . . 80

4 Conclusion $\quad 84$

List of References . . . . . . . . . . . . . . . . . . 85

Appendix A Dimensional Regularization Reference Formulae $\quad 86$

List of References . . . . . . . . . . . . . . . . . . . . . 88

Appendix B Derivation of Pion Decay Operator Anomalous Dimen$\begin{array}{ll}\text { sion } & 89\end{array}$

List of References . . . . . . . . . . . . . . . . . . . . . 93

$\begin{array}{ll}\text { Appendix C Fierz Transformations } & 94\end{array}$

List of References . . . . . . . . . . . . . . . . . . 97

Appendix D Computation of Supercompositeness Diagram $\quad 98$ 


\section{List of Tables}

3.1 Limits on RPV SUSY couplings from slepton exchange contribution . 65

3.2 Limits on RPV SUSY couplings from squark exchange contribution . 66

3.3 Leptoquark quantum numbers . . . . . . . . . . . . 68

3.4 Pseudoscalar leptoquark limits . . . . . . . . . . . . . . 70

3.5 Axial-vector leptoquark limits . . . . . . . . . . . 71 


\section{List of Figures}

$2.1 \phi^{4}$ propagator correction $\ldots \ldots \ldots \ldots \ldots \ldots \ldots \ldots \ldots$

$2.2 K-\bar{K}$ mixing in the Standard Model. . . . . . . . . . . . 47

$2.3 K-\bar{K}$ mixing contribution from $\Delta S=1$ operator . . . . . . . 48

$2.4 K-\bar{K}$ mixing contribution from $\Delta S=2$ operator . . . . . . . . 49

3.1 RPV SUSY slepton exchange contribution to pion decay . . . . . . 64

3.2 RPV SUSY squark exchange contribution to pion decay . . . . . . 66

3.3 Supercompositeness contribution to pion decay . . . . . . . . . 77

B.1 QCD correction to pseudoscalar pion decay operator $\ldots \ldots \ldots . .90$ 


\section{Chapter 1}

\section{The Standard Model}

\subsection{Introduction}

The Standard Model of particle physics describes all of the currently known elementary particles in nature and the forces that act between them, with the exception of gravity. The particles that make up matter, known as fermions, carry half-integral spin (intrinsic angular momentum), in units of $\hbar=\frac{h}{2 \pi}$, where $h$ is Planck's constant. Fermions interact by exchanging particles of integral spin, known as bosons. Bosons can also mediate interactions between themselves.

The electromagnetic force is carried by the photon, which has spin 1 . Since photons may only be emitted and absorbed by electrically charged particles, electrically neutral particles do not feel the electromagnetic force. The weak nuclear force is carried by the $W^{ \pm}$and $Z$ gauge bosons, which also have spin 1. All Standard Model particles have weak charge, and can exchange $W$ and $Z$ bosons. Finally, the strong nuclear force is carried by eight spin 1 gluons, which only couple to particles with colour charge. Colour charge is a quantum number analogous to electric charge, except that it comes in three different types; conventionally, these are taken to be red, blue, and green.

The two fundamental types of fermions are quarks and leptons. Quarks carry a 
unit of colour charge. That is, there exist red quarks, blue quarks, and green quarks. There are six different types, or flavours, of quarks: up $(u)$, down $(d)$, strange $(s)$, charm $(c)$, top $(t)$, and bottom $(b)$. The $u, c$, and $t$ quarks carry electric charge $+\frac{2}{3} e$, where $e$ is the absolute value of the electron charge. The $d, s$, and $b$ quarks carry electric charge $-\frac{1}{3} e$. Since quarks carry colour, they feel every force in the Standard Model. On the other hand, leptons are "colourless", so they do not feel the strong force. The six different types of leptons are the electron $(e)$, muon $(\mu)$, tau $(\tau)$, electron neutrino $\left(\nu_{e}\right)$, muon neutrino $\left(\nu_{\mu}\right)$, and tau neutrino $\left(\nu_{\tau}\right)$. The $e, \mu$, and $\tau$ leptons have charge $-e$, and the neutrinos are electrically neutral. Consequently, neutrinos only interact through the weak force.

The last component of the Standard Model is the Higgs doublet, which is a set of two complex spin 0 particles. Through spontaneous breaking of the electroweak symmetry of the Standard Model (see section 1.3), the $W$ and $Z$ bosons acquire masses by "eating" three of the four Higgs degrees of freedom. After electroweak symmetry breaking, then, only one degree of freedom is left in the Higgs sector. It is a real spin 0 particle, or scalar, and does not carry electric or colour charge. The Higgs boson is responsible for all particle masses in the Standard Model, and to date is the only undiscovered Standard Model particle.

In what follows, we review the mathematical framework of the Standard Model. More details on the construction, and properties of the Standard Model of particle physics may be found in the Particle Data Group summary [1], or in the standard texts $[2,3,4,5,6,7,8,9]$. After a general discussion of symmetries in field theories, we construct the Lagrangian of the Standard Model from its group structure. From the Lagrangian, the Feynman rules may be determined. Finally, we discuss renormalization in the Standard Model, demonstrating how its parameters change with energy. 


\subsection{Gauge Theory}

\subsubsection{Euler-Lagrange Equations of Motion}

The fundamental quantity in field theory is the Lagrangian density, denoted by $\mathcal{L}$. Usually, we will simply refer to $\mathcal{L}$ as the Lagrangian. The Lagrangian is a function of the fields and their covariant derivatives. We construct the action from the Lagrangian by integrating over spacetime,

$$
S=\int \mathcal{L} d^{4} x
$$

By Hamilton's principle for classical histories of the system, the action must be at an extremum. Therefore, $S$ must be unchanged for any infinitesimal change in the fields that vanishes at infinity. Consider a Lagrangian involving only one field $\phi$. By the chain rule, a change in $\phi$ yields a change in $\mathcal{L}\left(\phi, \partial_{\mu} \phi\right)$ of

$$
\delta \mathcal{L}=\frac{\partial \mathcal{L}}{\partial \phi} \delta \phi+\frac{\partial \mathcal{L}}{\partial\left(\partial_{\mu} \phi\right)} \delta\left(\partial_{\mu} \phi\right)
$$

Integrating by parts and using $\delta\left(\partial_{\mu} \phi\right)=\partial_{\mu}(\delta \phi)$ gives

$$
\delta \mathcal{L}=\frac{\partial \mathcal{L}}{\partial \phi} \delta \phi+\partial_{\mu}\left(\frac{\partial \mathcal{L}}{\partial\left(\partial_{\mu} \phi\right)} \delta \phi\right)-\partial_{\mu}\left(\frac{\partial \mathcal{L}}{\partial\left(\partial_{\mu} \phi\right)}\right) \delta \phi
$$

When integrated over spacetime to yield the change in the action $\delta S$, the middle term in the above expression will vanish, as it is a total derivative, and $\delta \phi$ goes to zero at infinity. The change in the action is thus

$$
\delta S=\int d^{4} x\left(\frac{\partial \mathcal{L}}{\partial \phi}-\partial_{\mu}\left(\frac{\partial L}{\partial\left(\partial_{\mu} \phi\right)}\right)\right) \delta \phi
$$


and since $\delta S$ must be zero regardless of the form of $\delta \phi$, the Euler-Lagrange equations of motion follow:

$$
\frac{\partial \mathcal{L}}{\partial \phi}=\partial_{\mu}\left(\frac{\partial \mathcal{L}}{\partial\left(\partial_{\mu} \phi\right)}\right)
$$

If there is more than one field in the Lagrangian, equation 1.5 holds for each of the fields separately, since for $S$ to be at an extremum, it must be unchanged under an infinitesimal variation of any one of the fields.

As an example, consider the Lagrangian

$$
\mathcal{L}=\frac{1}{2}\left(\partial_{\mu} \phi\right)^{2}-\frac{1}{2} m^{2} \phi^{2}
$$

where $\phi$ is a real scalar field. From equation $1.5, \phi$ must satisfy the equation of motion

$$
\left(\partial_{\mu} \partial^{\mu}+m^{2}\right) \phi=0
$$

which is just the Klein-Gordon equation.

\subsubsection{Noether's Theorem}

The equations of motion of a theory are often invariant under certain transformations of the fields, called symmetries. For the equations of motion to be invariant under a transformation, the Lagrangian may change by, at most, a pure divergence under the transformation, since this will leave the action unaffected. For example, all physical theories are invariant under Lorentz transformations. Noether's theorem relates symmetries to conservation laws. Suppose that under the transformation

$$
\phi \rightarrow \phi+\delta \phi
$$


the Lagrangian $\mathcal{L}\left(\phi, \partial_{\mu} \phi\right)$ changes by the divergence $\partial_{\mu} K^{\mu}$. Using the chain rule and the Euler-Lagrange equation 1.5, we may write the change in $\mathcal{L}$ as

$$
\begin{aligned}
\delta \mathcal{L} & =\frac{\partial \mathcal{L}}{\partial \phi} \delta \phi+\frac{\partial \mathcal{L}}{\partial\left(\partial_{\mu} \phi\right)} \delta\left(\partial_{\mu} \phi\right) \\
& =\partial_{\mu}\left(\frac{\partial \mathcal{L}}{\partial\left(\partial_{\mu} \phi\right)}\right) \delta \phi+\frac{\partial \mathcal{L}}{\partial\left(\partial_{\mu} \phi\right)} \partial_{\mu}(\delta \phi) \\
& =\partial_{\mu}\left(\frac{\partial \mathcal{L}}{\partial\left(\partial_{\mu} \phi\right)} \delta \phi\right)
\end{aligned}
$$

Equating this expression with $\partial_{\mu} K^{\mu}$, we find that

$$
\partial_{\mu} J^{\mu}=0
$$

where the conserved current is given by

$$
J^{\mu}=\frac{\partial \mathcal{L}}{\partial\left(\partial_{\mu} \phi\right)} \delta \phi-K^{\mu}
$$

If $\mathcal{L}$ is invariant under a transformation, then clearly $K^{\mu}=0$. The generalization to the case of multiple fields $\phi^{i}$ is also readily seen:

$$
J^{\mu}=\frac{\partial \mathcal{L}}{\partial\left(\partial_{\mu} \phi^{i}\right)} \delta \phi^{i}-K^{\mu}
$$

where summation is implied over $i$.

Consider translational invariance. Under the infinitesimal coordinate transformation

$$
x^{\mu} \rightarrow x^{\mu}+a^{\mu},
$$

the field $\phi(x)$ will change by an amount

$$
\delta \phi=a^{\mu} \partial_{\mu} \phi
$$


$\mathcal{L}$ transforms in the same manner as $\phi$, so in this case,

$$
K^{\mu}=a^{\mu} \mathcal{L}
$$

The conserved current is thus

$$
J^{\mu}=a_{\nu}\left(\frac{\partial \mathcal{L}}{\partial\left(\partial_{\mu} \phi\right)} \partial^{\nu} \phi-g^{\mu \nu} \mathcal{L}\right)
$$

In particular, since $a_{\nu}$ is arbitrary, the stress-energy tensor

$$
T^{\mu \nu}=\frac{\partial \mathcal{L}}{\partial\left(\partial_{\mu} \phi\right)} \partial^{\nu} \phi-g^{\mu \nu} \mathcal{L}
$$

satisfies $\partial_{\mu} T^{\mu \nu}=0$. Taking $\mu=\nu=0$ yields conservation of energy.

While the symmetries of the Standard Model are more abstract than translational invariance, Noether's theorem relates them to conservation laws in the same manner. For example, we will see that the Lagrangian of the Standard Model is invariant under a $U(1)$ phase transformation, which corresponds to conservation of electric charge.

\subsubsection{Gauging Symmetries}

The Lagrangian for a free complex scalar field is

$$
\mathcal{L}=\left|\partial_{\mu} \phi\right|^{2}-m^{2}|\phi|^{2}
$$

leading to the Klein-Gordon equation for both $\phi$ and $\phi^{*}$, which are separate degrees of freedom. Note that $\mathcal{L}$ is invariant under the phase rotation $\phi \rightarrow e^{i q \alpha} \phi$. Consider the infinitesimal version of this transformation,

$$
\phi \rightarrow \phi+i q \alpha \phi
$$


The conserved current associated with this symmetry is

$$
J^{\mu}=i \alpha\left(\phi \partial^{\mu} \phi^{*}\right)+\text { h.c. }
$$

If $q$ is taken to be the electric charge of the field $\phi$, then the conservation law implied by the symmetry is just the statement of electric current conservation.

So far, the symmetries considered have been global. That is, the same transformation has been applied at each point in spacetime. Now, we impose a local $U(1)$ symmetry, i.e. we allow the transformation parameter $\alpha$ to become a function of $x$. This procedure is known as gauging the symmetry. The $\partial_{\mu} \phi$ terms in the Lagrangian are affected. Instead of $\partial_{\mu} \phi \rightarrow \partial_{\mu} \phi+i q \alpha \partial_{\mu} \phi$, the new transformation law is

$$
\partial_{\mu} \phi \rightarrow \partial_{\mu} \phi+i q \alpha\left(\partial_{\mu} \phi\right)+i q\left(\partial_{\mu} \alpha\right) \phi
$$

and in general $\partial_{\mu} \alpha \neq 0$. To restore the invariance of $\mathcal{L}$, we replace the ordinary derivative $\partial_{\mu}$ by the covariant derivative

$$
D_{\mu}=\partial_{\mu}+i q A_{\mu}
$$

everywhere in the theory, where $A_{\mu}$ is a spin 1 field that transforms under a $U(1)$ phase rotation as

$$
A_{\mu} \rightarrow A_{\mu}-\partial_{\mu} \alpha
$$

The covariant derivative transforms as $D_{\mu} \phi \rightarrow(1+i q \alpha)\left(D_{\mu} \phi\right)$, and it follows that $\mathcal{L}$ is invariant under $U(1)$ transformations. Note that the introduction of $A^{\mu}$ modifies the equations of motion. $\phi$ and $\phi^{*}$ no longer satisfy the Klein-Gordon equation because they are not free fields anymore.

Now consider the equation of motion of $A^{\mu}$. After gauging the U(1) symmetry, it 
is

$$
i q \phi\left(\partial_{\mu} \phi^{*}\right)-i q\left(\partial_{\mu} \phi\right) \phi^{*}+2 q^{2} A_{\mu}|\phi|^{2}=0
$$

Since the left hand side is the potential for $A^{\mu}$ and appears to resemble the electric current of the original theory, we recall that the photon mediates the electomagnetic force and interpret $A^{\mu}$ as the photon field. If $A^{\mu}$ is to be a physical field in the theory, however, it must have a kinetic term (a term involving its derivative) in the Lagrangian, to describe propagation of photons in spacetime. Writing

$$
F^{\mu \nu}=\partial^{\mu} A^{\nu}-\partial^{\nu} A^{\mu}
$$

we add an appropriate term to the Lagrangian, yielding

$$
\mathcal{L}=\left|\partial_{\mu} \phi\right|^{2}-m^{2}|\phi|^{2}-\frac{1}{4} F^{\mu \nu} F_{\mu \nu}
$$

This kinetic term has been chosen so that the equation of motion for $A^{\mu}$ becomes

$$
i q \phi\left(\partial_{\mu} \phi^{*}\right)-i q\left(\partial_{\mu} \phi\right) \phi^{*}+2 q^{2} A_{\mu}|\phi|^{2}=\partial^{\nu} F_{\nu \mu}
$$

which is of the form of Maxwell's equations, $\partial^{\nu} F_{\nu \mu}=J_{\mu}$. There is now an additional term in the electric current, corresponding to a new interaction that resulted from gauging the $U(1)$ symmetry. $\mathcal{L}$ is in fact the Lagrangian for scalar quantum electrodynamics (QED), which describes the behaviour of charged spin 0 particles such as the $\pi^{ \pm}$mesons.

\subsubsection{Non-Abelian Gauge Groups}

The symmetry group in scalar QED is commutative, or Abelian; two $U(1)$ transformations applied successively produce the same result. regardless of the order in 
which they are applied. However, symmetry groups of field theories are, in general, not Abelian. An example of a non-Abelian symmetry group is $S U(N)$. In the Standard Model, the strong interaction is governed by $S U(3)$. In this section, we will describe Quantum Chromodynamics (QCD) in this section, which is the asymptotically free $[10,11,12,13,14] S U(3)$ gauge theory describing the interaction of quarks and gluons.

Since the quarks (and leptons) of the Standard Model are fermions, they are described by spinors that obey the Dirac equation. Defining

$$
\begin{aligned}
\sigma^{\mu} & =\left(1, \sigma^{i}\right) \\
\bar{\sigma}^{\mu} & =\left(1,-\sigma^{i}\right)
\end{aligned}
$$

where the $\sigma^{i}$ are the $2 \times 2$ Pauli matrices and 1 is the $2 \times 2$ identity matrix, we take the gamma matrices to be

$$
\gamma^{\mu}=\left(\begin{array}{cc}
0 & \sigma^{\mu} \\
\bar{\sigma}^{\mu} & 0
\end{array}\right)
$$

in the Weyl basis. Note that in this basis, the product of the four gamma matrices is proportional to

$$
\gamma^{5}=i \gamma^{0} \gamma^{1} \gamma^{2} \gamma^{3}=\left(\begin{array}{cc}
-1 & 0 \\
0 & 1
\end{array}\right),
$$

and we can break a spinor into its chiral (left- and right-handed) components using $\gamma^{5}$ :

$$
\begin{aligned}
\psi_{L} & =\frac{1-\gamma^{5}}{2} \psi \\
\psi_{R} & =\frac{1+\gamma^{5}}{2} \psi
\end{aligned}
$$


The conjugate of a spinor $\psi$ is given by

$$
\bar{\psi}=\psi^{\dagger} \gamma^{0}
$$

The free Lagrangian for a spinor is

$$
\mathcal{L}=\bar{\psi}\left(i \gamma^{\mu} \partial_{\mu}-m\right) \psi
$$

from which the Dirac equation

$$
\left(i \gamma^{\mu} \partial_{\mu}-m\right) \psi=0
$$

follows.

In the $S U(3)$ theory, spinors of red, blue, and green quarks will transform into each other without affecting the equations of motion. We will use Latin letters for group indices, so that $\psi^{i}$, for $1 \leq i \leq 3$ are the spinors for the diffently coloured quarks. The free Lagrangian for the spinors $\psi^{i}$ is

$$
\mathcal{L}=\bar{\psi}^{i}\left(i \gamma^{\mu} \partial_{\mu}-m\right) \psi^{i}
$$

where summation over $i$ is implied. In particular, $\mathcal{L}$ is symmetric under the $S U(3)$ transformation

$$
\psi^{i} \rightarrow\left[e^{\left(i g \alpha^{a} T^{a}\right)}\right]^{i j} \psi^{j}
$$

where the $T^{a}$ are $3 \times 3$ matrices which form a representation of the generators of the $S U(3)$ algebra, the $\alpha^{a}$ are arbitrary parameters of the transformation, and summation over $a$ is implied. The $S U(3)$ algebra is given by

$$
\left[T^{a}, T^{b}\right]=i f^{a b c} T^{c}
$$


with the structure constants

$$
\begin{gathered}
f^{123}=1 \\
f^{147}=f^{246}=f^{257}=f^{345}=-f^{156}=-f^{367}=\frac{1}{2} \\
f^{458}=f^{678}=\frac{\sqrt{3}}{2} .
\end{gathered}
$$

All of the other $f^{a b c}$ are zero. Since the structure constants satisfy a commutation relation, they are antisymmetric in their first two indices. A particular choice for the $T^{a}$ is provided by the Gell-Mann matrices, since we may take

$$
T^{a}=\frac{1}{2} \lambda^{a}
$$

where the Gell-Mann matrices $\lambda^{a}$ are

$$
\begin{aligned}
& \lambda^{1}=\left(\begin{array}{ccc}
0 & 1 & 0 \\
1 & 0 & 0 \\
0 & 0 & 0
\end{array}\right), \lambda^{2}=\left(\begin{array}{ccc}
0 & -i & 0 \\
i & 0 & 0 \\
0 & 0 & 0
\end{array}\right), \lambda^{3}=\left(\begin{array}{ccc}
1 & 0 & 0 \\
0 & -1 & 0 \\
0 & 0 & 0
\end{array}\right), \lambda^{4}=\left(\begin{array}{ccc}
0 & 0 & 1 \\
0 & 0 & 0 \\
1 & 0 & 0
\end{array}\right), \\
& \lambda^{5}=\left(\begin{array}{ccc}
0 & 0 & -i \\
0 & 0 & 0 \\
i & 0 & 0
\end{array}\right), \lambda^{6}=\left(\begin{array}{ccc}
0 & 0 & 0 \\
0 & 0 & 1 \\
0 & 1 & 0
\end{array}\right), \lambda^{7}=\left(\begin{array}{ccc}
0 & 0 & 0 \\
0 & 0 & -i \\
0 & i & 0
\end{array}\right), \lambda^{8}=\frac{1}{\sqrt{3}}\left(\begin{array}{ccc}
1 & 0 & 0 \\
0 & 1 & 0 \\
0 & 0 & -2
\end{array}\right) .
\end{aligned}
$$

Note that in this basis, the structure constants are antisymmetric in all of their indices, not just the first two. 
We now gauge the $S U(3)$ symmetry by allowing the $\alpha^{a}$ to be functions of spacetime, $\alpha^{a}(x)$. Under the infinitesimal version of the local symmetry,

$$
\psi^{i} \rightarrow \psi^{i}+i g\left[\alpha^{a} T^{a}\right]^{i j} \psi^{j}
$$

While the $\bar{\psi} \psi$ term of $\mathcal{L}$ is still invariant under the local symmetry, the derivative term now transforms non-trivially, since

$$
\partial_{\mu} \psi^{i} \rightarrow \partial_{\mu} \psi^{i}+i g\left[\alpha^{a} T^{a}\right]^{i j} \partial_{\mu} \psi^{j}+i g\left[\left(\partial_{\mu} \alpha^{a}\right) T^{a}\right]^{i j} \psi^{j}
$$

Just as in the Abelian case, the symmetry may be restored by replacing $\partial_{\mu}$ with the covariant derivative $D_{\mu}$. Since there are multiple generators of the symmetry group, we generalize the $U(1)$ case to obtain

$$
D_{\mu}=\partial_{\mu}+i g b_{\mu}^{a} T^{a}
$$

where the $b^{a}$ are a set of eight spin 1 fields. We can obtain the transformation law for the $b_{\mu}^{a} T^{a}$ by writing

$$
\begin{aligned}
D_{\mu} \psi^{i} & \rightarrow \partial_{\mu}\left(\left(1+i g \alpha^{a} T^{a}\right)^{i j} \psi^{j}\right)+i g\left(b_{\mu}^{\prime a} T^{a}\right)^{i j}\left(1+i g \alpha^{b} T^{b}\right)^{j k} \psi^{k} \\
& =\left(1+i g \alpha^{a} T^{a}\right)^{i j}\left(\partial_{\mu} \psi^{j}\right)+i g\left(\left(\partial_{\mu} \alpha^{a}\right) T^{a}\right)^{i j} \psi^{j}+i g\left(b_{\mu}^{\prime a} T^{a}\right)^{i j}\left(1+i g \alpha^{b} T^{b}\right)^{j k} \psi^{k}
\end{aligned}
$$

where $b_{\mu}^{\prime a}$ denotes the transformed $b^{a}$. If we require that the covariant derivative satisfy the same transformation law as before,

$$
D_{\mu} \psi^{i} \rightarrow\left(1+i g \alpha^{a} T^{a}\right)^{i j}\left(D_{\mu} \psi^{j}\right)=\left(1+i g \alpha^{a} T^{a}\right)^{i j}\left(\partial_{\mu} \psi^{j}+i g\left(b_{\mu}^{b} T^{b}\right)^{j k} \psi^{k}\right)
$$


we have the constraint

$$
\left(1+i g \alpha^{a} T^{a}\right)^{i j}\left(i g\left(b_{\mu}^{b} T^{b}\right)^{j k} \psi^{k}\right)=i g\left(\left(\partial_{\mu} \alpha^{a}\right) T^{a}\right)^{i j} \psi^{j}+i g\left(b_{\mu}^{a} T^{a}\right)^{i j}\left(1+i g \alpha^{b} T^{b}\right)^{j k} \psi^{k}
$$

If this is to hold for all possible spinors $\psi^{i}$, then we may rearrange and multiply on the left by $\left(1+i g \alpha^{a} T^{a}\right)^{-1}=1-i g \alpha^{a} T^{a}$ to obtain

$$
\left(b_{\mu}^{\prime} T^{a}\right)^{i j}=\left(b_{\mu}^{a} T^{a}\right)^{i j}+i g \alpha^{a} b_{\mu}^{b}\left[T^{a}, T^{b}\right]^{i j}-\left(\left(\partial_{\mu} \alpha^{a}\right) T^{a}\right)^{i j}
$$

where we have neglected terms of order $\alpha^{2}$. Using the commutation relation of the $T^{a}$ provided by the $S U(3)$ algebra, the final transformation law for the $b_{\mu}^{a} T^{a}$ is

$$
\left(b_{\mu}^{a} T^{a}\right)^{i j} \rightarrow\left(b_{\mu}^{a} T^{a}\right)^{i j}-g f^{a b c} \alpha^{a} b_{\mu}^{b}\left(T^{c}\right)^{i j}-\left(\left(\partial_{\mu} \alpha^{a}\right) T^{a}\right)^{i j}
$$

From here on, we will suppress group indices and use the dot product to identify quantities that are summed over them. For instance, defining $B_{\mu} \equiv b_{\mu} \cdot T$, we have the transformation law

$$
B_{\mu} \rightarrow B_{\mu}+i g\left[\alpha \cdot T, B_{\mu}\right]-\left(\partial_{\mu} \alpha\right) \cdot T
$$

In non-Abelian gauge theory, the gauge boson kinetic term is slightly more complicated. Its specific form may be derived by requiring that the field strength tensor transform in the same way as $F_{\mu \nu}$ does in QED. We omit the details, and simply state the result

$$
G_{\mu \nu}^{a}=\partial_{\mu} B_{\nu}^{a}-\partial_{\nu} B_{\mu}^{a}-g f^{a b c} B_{\mu}^{b} B_{\nu}^{c}
$$

leading to the final Lagrangian

$$
\mathcal{L}=\bar{\psi}\left(i \gamma^{\mu} D_{\mu}-m\right) \psi-\frac{1}{4} G^{\mu \nu} G_{\mu \nu}
$$


Note that by expanding the kinetic term, we obtain interaction terms involving three or four gauge bosons. These interactions are a new feature of non-Abelian gauge theory, and arise because the gauge bosons themselves carry the gauge charge. While the photon does not carry any electric charge, gluons carry colour charge. For example, a gluon may carry one unit of red charge and one unit of anti-blue charge. These types of interactions also occur in the electroweak theory, as its gauge group includes the non-Abelian $S U(2)$.

\subsection{Spontaneous Symmetry Breaking}

In pure gauge theories, boson mass terms, which are proportional to $A^{\mu} A_{\mu}$, are forbidden by gauge invariance. For example, consider scalar QED. Applying the photon transformation law, we find that

$$
A^{\mu} A_{\mu} \rightarrow\left(A^{\mu}-\partial^{\mu} \alpha\right)\left(A_{\mu}-\partial_{\mu} \alpha\right)=A^{\mu} A_{\mu}-2 A^{\mu}\left(\partial_{\mu} \alpha\right)
$$

i.e., the $A^{2}$ term is not invariant under a $U(1)$ transformation.

However, experimental evidence shows that massive gauge bosons exist. Therefore, in order to write the Standard Model as a gauge theory, we need a mechanism through which gauge bosons may acquire mass. Spontaneous symmetry breaking provides this mechanism $[15,16,17,18,19,20,21]$, and is the final tool we need to write down the Standard Model Lagrangian. 


\subsection{1 $\phi^{4}$ Theory}

To introduce spontaneously broken symmetries, we consider the theory of a real scalar field $\phi$ with quadratic and quartic terms in the potential. The Lagrangian is

$$
\mathcal{L}=\frac{1}{2}\left(\partial^{\mu} \phi\right)\left(\partial_{\mu} \phi\right)-\frac{1}{2} \mu^{2} \phi^{2}-\frac{\lambda}{4 !} \phi^{4}
$$

where $\mu^{2}$ and $\lambda$ are parameters of the theory. The potential for $\phi$ is

$$
V(\phi)=\frac{1}{2} \mu^{2} \phi^{2}+\frac{\lambda}{4 !} \phi^{4}
$$

Note that $\mathcal{L}$ is symmetric under a discrete parity symmetry, $\phi \rightarrow-\phi$. We will find the vacuum state by solving for the value of $\phi$ which minimizes the potential. Then, we may expand the potential about the vacuum state to obtain the perturbations that give rise to interactions. The use of $\mu^{2}$ instead of $\mu$ as a parameter is purely historical, and we will look at both the cases $\mu^{2}>0$ and $\mu^{2}<0$. However, we take $\lambda>0$, because if $\lambda$ is negative, the potential has no minimum.

Suppose $\mu^{2}>0$. Then the minimum of the potential is clearly at $\phi=0$. Expanding around this minimum, we trivially find

$$
\mathcal{L}=\frac{1}{2}\left(\partial^{\mu} \phi\right)\left(\partial_{\mu} \phi\right)-\frac{1}{2} \mu^{2} \phi^{2}-\frac{\lambda}{4 !} \phi^{4}
$$

which is just the original Lagrangian. This is a theory of a massive real scalar with a quartic interaction.

Now, suppose $\mu^{2}<0$. Solving for the minimum of $V(\phi)$ yields

$$
\langle\phi\rangle= \pm \sqrt{\frac{-6 \mu^{2}}{\lambda}}
$$

where $\langle\phi\rangle$ is the vacuum expectation value of $\phi$. There are two distinct possible 
vacuum states, and we must choose one as our minimum. Without loss of generality, we take the positive solution. Now, we expand around $\langle\phi\rangle$ by writing $\phi=\phi^{\prime}+\langle\phi\rangle$, yielding

$$
\mathcal{L}=\frac{1}{2}\left(\partial^{\mu} \phi^{\prime}\right)\left(\partial_{\mu} \phi^{\prime}\right)+\mu^{2} \phi^{2}-\frac{\lambda}{3 !}\langle\phi\rangle \phi^{\prime 3}-\frac{\lambda}{4 !} \phi^{\prime 4}
$$

where we have omitted a constant term that does not affect the equations of motion. Note that the mass of the scalar has changed from $\left|\mu^{2}\right|$ to $\sqrt{2}\left|\mu^{2}\right|$. As well, although the linear term cancels, the cubic term does not, and so the Lagrangian is not symmetric under $\phi^{\prime} \rightarrow-\phi^{\prime}$. The parity symmetry of $\mathcal{L}$ has been broken by expanding around a particular vacuum expectation value for $\phi$.

Spontaneous symmetry breaking leads to new interactions in a theory. In $\phi^{4}$ theory, a cubic interaction is now present in $\mathcal{L}$. We will see that the breaking of a continuous symmetry can have even greater effects.

\subsubsection{Electroweak theory}

Glashow, Weinberg, and Salam used the Higgs mechanism to develop a unified theory of the electromagnetic and weak interactions [22, 23, 24]. In the GWS theory, an $S U(2) \times U(1)$ symmetry is spontaneously broken by the vacuum expectation value of the Higgs field, leaving only a $U(1)$ symmetry. We start with a set of spinors $\psi^{i}$, where $1 \leq i \leq 2$, representing massless particles that obey the Dirac equation

$$
i \gamma^{\mu} \partial_{\mu} \psi^{i}=0
$$

In the GWS theory, the doublet is composed of two left-handed spinors. The lepton doublets are

$$
\left(\begin{array}{l}
\nu_{l} \\
l
\end{array}\right)_{L}
$$


where $l$ can be $e, \mu$, or $\tau$. The subscript $L$ refers to the fact that only the left-handed pieces of the spinors are in the doublet. The quark doublets are

$$
\left(\begin{array}{c}
u_{a} \\
d_{a}
\end{array}\right)_{L},\left(\begin{array}{c}
c_{a} \\
s_{a}
\end{array}\right)_{L},\left(\begin{array}{c}
t_{a} \\
b_{a}
\end{array}\right)_{L}
$$

where the colour index $a$ can be $r, b$, or $g$. By analogy with spin in quantum mechanics, we refer to these doublets as weak isospin doublets, and assign the top and bottom members of a doublet $I_{3}=\frac{1}{2}$ and $I_{3}=-\frac{1}{2}$, respectively. We say that the doublet carries isospin $I=\frac{1}{2}$. This nomenclature is completely analogous to the use of spin doublets to represent spin-up and spin-down states of an electron.

The Lagrangian

$$
\mathcal{L}=\bar{\psi}\left(i \gamma^{\mu} \partial_{\mu}\right) \psi
$$

is invariant under both $U(1)$ and $S U(2)$ transformations (we continue to suppress the group indices on $\psi$ ). Gauging both symmetries and adding the kinetic terms for the new gauge bosons gives

$$
\mathcal{L}=\bar{\psi}\left(i \gamma^{\mu} D_{\mu}\right) \psi-\frac{1}{4} F^{\mu \nu} F_{\mu \nu}-\frac{1}{4} W^{\mu \nu} W_{\mu \nu}
$$

where the covariant derivative is

$$
D_{\mu}=\partial_{\mu}+i \frac{g^{\prime}}{2} B_{\mu} Y+i g W_{\mu}
$$

with $B_{\mu}$ and $W_{\mu}$ being the gauge bosons associated with the $U(1)$ and $S U(2)$ symmetries, respectively, and $F_{\mu \nu}$ and $W_{\mu \nu}$ the corresponding field strength tensors. The factors of 2 are purely conventional. Note that $W_{\mu}=W_{\mu}^{a} \frac{\sigma^{a}}{2}$, where $1 \leq a \leq 3$ and the $\sigma^{a}$ are the Pauli matrices. $Y$ is the hypercharge, and is different for quark doublets 
than for lepton doublets, while $g^{\prime}$ and $g$ are coupling constants associated with the symmetry groups. By imposing the Gell-Mann-Nishijima relation [25, 26],

$$
Q=I_{3}+\frac{1}{2} Y
$$

we may determine the hypercharge of each doublet. Each lepton doublet has hypercharge -1 , and each quark doublet has hypercharge $\frac{1}{3}$.

The GWS theory includes the right-handed spinors as weak isospin singlets. That is, we add the term

$$
\mathcal{L} \supset \bar{\eta}\left(i \gamma^{\mu} \partial_{\mu}\right) \eta
$$

where we sum over all the possible right-handed spinors $\eta$. The leptonic right-handed spinors are $e_{R}, \mu_{R}$, and $\tau_{R}$, and the quark right-handed spinors are $u_{R}^{a}, d_{R}^{a}, c_{R}^{a}, s_{R}^{a}$, $t_{R}^{a}$, and $b_{R}^{a}$, where $a$ takes the same possible values as before. Note that every lefthanded spinor has a right-handed counterpart, except for the neutrinos. Since mass terms combine the left-handed and right-handed components of spinors, all massive particles must exist in both chiralities. However, the Standard Model takes neutrinos to be massless, and so no right-handed neutrino fields are required. The Lagrangian terms with right-handed spinors are trivially invariant under $S U(2)$ transformations, since their $S U(2)$ representations are trivial. However, they are also invariant under $U(1)$ phase rotations. When the symmetries are gauged, the derivative in these terms is thus replaced by a covariant derivative

$$
D_{\mu}=\partial_{\mu}+i \frac{g^{\prime}}{2} B_{\mu} Y
$$

Now, mass terms for the $B$ and $W$ gauge bosons are ordinarily forbidden by gauge invariance. But if a symmetry is spontaneously broken, we will see that gauge bosons may acquire mass. Although $\mathcal{L}$ is invariant under $S U(2) \times U(1)$ transformations, we 
can add a complex scalar doublet to the theory to break some of the symmetry. We thus introduce a new doublet of complex scalar fields

$$
\phi=\left(\begin{array}{c}
\phi^{+} \\
\phi^{0}
\end{array}\right),
$$

and note that by the charges of the component fields, $\phi$ must have hypercharge 1. We will add kinetic and potential terms for $\phi$ to the Lagrangian. The kinetic term is simply $\left(D_{\mu} \phi\right)^{\dagger}\left(D^{\mu} \phi\right)$, where $D_{\mu}$ is the same covariant derivative as for the left-handed spinors (because $\phi$ transforms non-trivially under both $S U(2)$ and $U(1)$ transformations). The minimal choice for the potential that provides spontaneous symmetry breaking but respects the $S U(2) \times U(1)$ invariance of the theory is

$$
V(\phi)=\mu^{2}\left(\phi^{\dagger} \phi\right)+\lambda\left(\phi^{\dagger} \phi\right)^{2}
$$

where we assume that the parameters of the potential satisfy $\mu^{2}<0$ and $\lambda>0$. Under these assumptions, $\phi$ acquires a non-zero vacuum expectation value that must satisfy

$$
\left\langle\phi^{\dagger} \phi\right\rangle=-\frac{\mu^{2}}{2 \lambda}
$$

In $\phi^{4}$ theory, there were a finite number of choices for the vacuum expectation value, since the broken symmetry was discrete. Here, we instead have an infinite number of possible choices, all related to each other by $S U(2) \times U(1)$ transformations. To preserve conservation of electromagnetic charge, the vacuum expectation value of the top component of $\phi$, which is charged, must be zero. There is still a $U(1)$ phase choice 
for the vacuum expectation value of the bottom component, and we choose

$$
\langle\phi\rangle=\left(\begin{array}{c}
0 \\
v / \sqrt{2}
\end{array}\right)
$$

where the minimization of the potential has required us to take

$$
v=\sqrt{-\frac{\mu^{2}}{\lambda}}
$$

Just as before, we now expand around the minimum. We may take the parameters of our expansion to be a set of real scalars $\rho^{a}$, where $1 \leq a \leq 3$, and the real scalar field $h$, after performing a $U(1)$ gauge transformation to remove the latter's phase. We write

$$
\phi=e^{i \frac{\rho \cdot \sigma}{v}}\left(\begin{array}{c}
0 \\
(v+h) / \sqrt{2}
\end{array}\right) .
$$

Note that the number of degrees of freedom in $\phi$ has been preserved. Originally, $\phi$ had two complex scalar components which made up four (real) degrees of freedom. Now, we have written $\phi$ in terms of four real scalars, so our expansion also has four degrees of freedom. Since the $S U(2)$ symmetry has been gauged, we may also immediately perform a local $S U(2)$ transformation to remove the $\rho$ fields without affecting the Lagrangian, giving

$$
\phi=\left(\begin{array}{c}
0 \\
(v+h) / \sqrt{2}
\end{array}\right) .
$$

This transformation chooses the gauge known as unitarity gauge. We now substitute this expansion for $\phi$ into the terms in the Lagrangian involving the scalar field. The 
relevant part of $\mathcal{L}$ becomes

$$
\begin{aligned}
\mathcal{L} & \supset\left(D_{\mu} \phi\right)^{\dagger}\left(D^{\mu} \phi\right)-\mu^{2}\left(\phi^{\dagger} \phi\right)-\lambda\left(\phi^{\dagger} \phi\right)^{2} \\
& \left.=\left|\left(\partial_{\mu}+i \frac{g^{\prime}}{2} B_{\mu} Y+i g W_{\mu}\right)\right| \begin{array}{c}
0 \\
(v+h) / \sqrt{2}
\end{array}\right)\left.\right|^{2}-\frac{1}{2} \mu^{2}(v+h)^{2}-\frac{1}{4} \lambda(v+h)^{4} \\
& =\frac{1}{2}\left(\partial_{\mu} h\right)^{2}+\left|\left(\frac{g^{\prime}}{2} B_{\mu} I+g W_{\mu}^{a} \frac{\sigma^{a}}{2}\right)\left(\begin{array}{c}
0 \\
(v+h) / \sqrt{2}
\end{array}\right)\right|^{2}-\frac{1}{2} \mu^{2}(v+h)^{2}-\frac{1}{4} \lambda(v+h)^{4},
\end{aligned}
$$

where $I$ is the $2 \times 2$ unit matrix. This expression contains a potential for the $h$ field, mass terms for the gauge bosons, and interaction terms among the gauge bosons and $h$.

The Higgs potential is formed by the non-kinetic terms involving only $h$, and is

$$
\mathcal{L} \supset-\frac{1}{4} \lambda h^{4}-\lambda v h^{3}+\mu^{2} h^{2}
$$

where we have omitted additive constants arising from terms involving only $v$. Remembering that $\mu^{2}<0$, we see that $h$ has a mass of $\sqrt{-2 \mu^{2}}$, and a potential involving both cubic and quartic terms.

The gauge boson mass and interaction terms come from the terms involving $B_{\mu}$ 
and $W_{\mu}^{a}$. Using the Pauli matrices explicitly yields

$$
\begin{aligned}
\left|\left(\frac{g^{\prime}}{2} B_{\mu} I+W_{\mu}^{a} \frac{\sigma^{a}}{2}\right)\left(\begin{array}{c}
0 \\
\frac{v+h}{\sqrt{2}}
\end{array}\right)\right|^{2} & =\left|\frac{1}{2}\left(\begin{array}{cc}
g^{\prime} B_{\mu}+g W_{\mu}^{3} & g\left(W_{\mu}^{1}-i W_{\mu}^{2}\right. \\
g\left(W_{\mu}^{1}+i W_{\mu}^{2}\right) & g^{\prime} B_{\mu}-g W_{\mu}^{3}
\end{array}\right)\left(\begin{array}{c}
0 \\
\frac{v+h}{\sqrt{2}}
\end{array}\right)\right|^{2} \\
& =(v+h)^{2}\left(\frac{g^{2}}{4}\left(W^{\mu+} W_{\mu}^{-}\right)+\frac{1}{8}\left(g^{\prime} B_{\mu}-g W_{\mu}^{3}\right)^{2}\right),
\end{aligned}
$$

where we have taken the conventional definition

$$
W_{\mu}^{ \pm}=\frac{W_{\mu}^{1} \mp i W_{\mu}^{2}}{\sqrt{2}}
$$

for the electrically charged $S U(2)$ gauge bosons. The terms that are quadratic in $v$ lead to mass terms for the gauge bosons. The $W^{ \pm}$bosons have mass

$$
m_{W}=\frac{g v}{2}
$$

and if we define the photon field $A$ and the $Z$ boson by

$$
\begin{aligned}
\left(\begin{array}{c}
Z_{\mu} \\
A_{\mu}
\end{array}\right) & =\frac{1}{\sqrt{g^{2}+g^{\prime 2}}}\left(\begin{array}{cc}
g & -g^{\prime} \\
g^{\prime} & g
\end{array}\right)\left(\begin{array}{l}
W_{\mu}^{3} \\
B_{\mu}
\end{array}\right) \\
& \equiv\left(\begin{array}{cc}
\cos \theta_{W} & -\sin \theta_{W} \\
\sin \theta_{W} & \cos \theta_{W}
\end{array}\right)\left(\begin{array}{l}
W_{\mu}^{3} \\
B_{\mu}
\end{array}\right),
\end{aligned}
$$

where $\theta_{W}$ is the weak mixing angle, the $Z$ boson acquires a mass as well, of

$$
m_{Z}=\frac{\sqrt{g^{2}+g^{2}} v}{2}
$$


The $W$ and $Z$ masses are thus related by $m_{W}=m_{Z} \cos \theta_{W}$. This relation is a sensitive test of the Standard Model, as both masses have been measured with high precision at colliders. Also, note that once the $S U(2) \times U(1)$ symmetry is broken, there is nothing to prevent gauge bosons of the same electric charge from mixing, and this is exactly what we have seen. The interactions between $h$ and the gauge bosons come from the other terms in equation 1.83. They are

$$
\mathcal{L} \supset\left(2 v h+h^{2}\right)\left(\frac{g^{2}}{4}\right)\left(W^{\mu+} W_{\mu}^{-}+\frac{1}{2 \cos ^{2} \theta_{W}} Z^{\mu} Z_{\mu}\right)
$$

To obtain the remaining interactions in the GWS theory, we would simply expand the terms in the Lagrangian. Note that since $S U(2)$ is a non-Abelian gauge group, the expansion of the kinetic term for the $W$ bosons yields three and four $W$ interactions. In the more physical basis of $W^{ \pm}, Z$, and $A$, these become interactions involving three or four of any of the gauge bosons, since $Z$ and $A$ both include parts of $W_{\mu}^{3}$. To reproduce the desired coupling structure in the neutral currents, we would expand the terms in the covariant derivative corresponding to the $A$ and $Z$ coupling to fermions. We find that, as desired, the photon couples to left-handed and right-handed spinors equally. As well, the electromagnetic interaction has strength $e$ if we require that

$$
e=\frac{g g^{\prime}}{\sqrt{g^{2}+g^{\prime 2}}}=g \sin \theta_{W}
$$

Finally, we turn to the question of fermion masses. In the GWS theory, lepton masses are provided by the interaction terms

$$
\mathcal{L} \supset-G_{\psi}\left(\left(\bar{\psi}_{L} \phi\right) \psi_{R}+\bar{\psi}_{R}\left(\phi^{\dagger} \psi_{L}\right)\right)
$$

where $\psi$ can be $e, \mu$, or $\tau$, and $G_{\psi}$ is the Yukawa coupling of $\psi$. Note that $\psi_{L}$ refers to the doublet consisting of the left-handed charged lepton together with its associated 
neutrino, while $\psi_{R}$ is the electroweak singlet of the right-handed charged lepton only. After spontaneous symmetry breaking, there are interaction terms proportional to $h \bar{\psi} \psi$, and effective mass terms for the lower components of each doublet. Expanding the term involving the electron doublet yields

$$
\mathcal{L} \supset-G_{e}\left(\frac{v+h}{\sqrt{2}}\right)\left(\bar{e}_{L} e_{R}+\bar{e}_{R} e_{L}\right)=-G_{e}\left(\frac{v+h}{\sqrt{2}}\right)(\bar{e} e)
$$

which indicates that the electron has acquired a mass of

$$
m_{e}=G_{e} \frac{v}{\sqrt{2}}
$$

The muon and tau leptons obtain mass in a similar fashion. Note that the neutrinos remain massless, as desired. (Massive neutrinos are outside the scope of the Standard Model.)

Quark masses are slightly more complicated, because in general, there can be a unitary matrix that mixes the weak interaction eigenstates and the mass eigenstates. In the leptonic sector, this issue does not arise because the neutrinos are massless. Writing the left-handed quark doublets as $Q_{i}, 1 \leq i \leq 3$ being the generation index, the Yukawa interaction terms are

$$
\mathcal{L} \supset-Y_{i j}^{d}\left(\bar{Q}_{i} \phi\right) d_{j}-Y_{i j}^{u}\left(\bar{Q}_{i}\left(i \sigma^{2}\right) \phi^{*}\right) u_{j}
$$

where $Y^{u}$ and $Y^{d}$ are the $3 \times 3$ matrices of Yukawa couplings, and $u_{i}, d_{i}$ are the righthanded quark spinors. For example, $u_{1}=\frac{1+\gamma^{5}}{2} u$, where $u$ is the ordinary up quark spinor. Note that the right-handed spinors are electroweak singlets, so that the $Q$ field always couples to $\phi$. After spontaneous symmetry breaking, the quarks acquire mass terms. The matrices $Y^{u}$ and $Y^{d}$ may be diagonalized, at the price of introducing a mixing matrix into the weak interaction eigenstates. The left-handed doublets on 
which the weak interactions now interact are $\left(\begin{array}{c}u \\ d^{\prime}\end{array}\right),\left(\begin{array}{c}c \\ s^{\prime}\end{array}\right),\left(\begin{array}{c}t \\ b^{\prime}\end{array}\right)$, where the weak eigenstates are related to the mass eigenstates by the Cabibbo-Kobayashi-Maskawa (CKM) matrix, $V$ :

$$
\left(\begin{array}{l}
d^{\prime} \\
s^{\prime} \\
b^{\prime}
\end{array}\right)=\left(\begin{array}{ccc}
V_{u d} & V_{u s} & V_{u b} \\
V_{c d} & V_{c s} & V_{c b} \\
V_{t d} & V_{t s} & V_{t b}
\end{array}\right)\left(\begin{array}{l}
d \\
s \\
b
\end{array}\right) .
$$

In practice, the CKM matrix is nearly diagonal, particularly in the entries corresponding to the top quark. Tests of the unitary of the CKM matrix serve as probes of physics beyond the Standard Model.

As the GWS theory provides a full description of the electromagnetic and weak forces, all that remains to construct the Standard Model is to include QCD through the introduction of an $S U(3)$ gauge symmetry. QCD has already been introduced as an example of a non-Abelian gauge theory, and we now incorporate it into the Standard Model.

\subsection{The Standard Model Lagrangian}

\subsection{1 $S U(3) \times S U(2) \times U(1)$}

Including the $S U(3)$ gauge group of the quarks in addition to the GWS theory, we have the following particles in the Standard Model:

- Four $S U(2) \times U(1)$ gauge bosons $W^{a}, 1 \leq a \leq 3$ and $B$, which are reexpressed as $W^{ \pm}, Z$, and $A$ after spontaneous symmetry breaking

- Eight gluons $G^{a}, 1 \leq a \leq 8$, the gauge bosons for the $S U(3)$ symmetry of the 
quarks

- The Higgs electroweak doublet $\phi$, which is responsible for breaking $S U(2) \times U(1)$ to the $U(1)$ of electromagnetism

- Three left-handed leptonic electroweak doublets $L_{L}$ that are $S U(3)$ singlets

- Three right-handed leptonic fields $l_{R}$, the chiral partners of the bottom components of the $L_{L}$, which are singlets under both $S U(2)$ and $S U(3)$

- Three left-handed quark fields $Q_{L}$ that are $S U(2)$ doublets and $S U(3)$ triplets

- Six right-handed quark fields $q_{R}$ that are $S U(2)$ singlets but $S U(3)$ triplets, the partners of the $Q_{L}$

The covariant derivative on each fermion spinor is different, because different fields do not transform in the same way under $S U(3) \times S U(2) \times U(1)$. The covariant derivative of a field that transforms as the fundamental representation under all of the gauge groups is given by

$$
D_{\mu}=\partial_{\mu}+i \frac{g^{\prime}}{2} B_{\mu} Y+i g W_{\mu}+i g_{s} G_{\mu},
$$

where the hypercharge $Y$ is given by the Gell-Mann-Nishijima relation, and $g_{s}$ is the $S U(3)$ (strong) coupling constant. For fields that transform as singlets under some of the groups, we simply omit the corresponding terms in the covariant derivative, as before.

We denote the $U(1), S U(2)$, and $S U(3)$ field-strength tensors by $F_{\mu \nu}, W_{\mu \nu}$, and $G_{\mu \nu}$, respectively. The full Lagrangian for the Standard Model is

$$
\begin{aligned}
\mathcal{L} & =i \bar{\psi} \gamma^{\mu} D_{\mu} \psi-\frac{1}{4} F^{\mu \nu} F_{\mu \nu}-\frac{1}{4} W^{\mu \nu} W_{\mu \nu}-\frac{1}{4} G^{\mu \nu} G_{\mu \nu} \\
& +\left(D_{\mu} \phi\right)^{\dagger}\left(D^{\mu} \phi\right)-\mu^{2}\left(\phi^{\dagger} \phi\right)-\lambda\left(\phi^{\dagger} \phi\right)^{2} \\
& -G_{l}\left(\left(\bar{L}_{L} \phi\right) l_{R}+\bar{l}_{R}\left(\phi^{\dagger} L_{L}\right)\right)-Y_{i j}^{d}\left(\left(\bar{Q}_{L}\right)_{i} \phi\right)\left(d_{R}\right)_{j}-Y_{i j}^{u}\left(\left(\bar{Q}_{L}\right)_{i}\left(i \sigma^{2}\right) \phi^{*}\right) u_{j},
\end{aligned}
$$


where the first term is a sum over all fermionic fields $\psi$, with the appropriate covariant derivative in each term. We have suppressed all indices except for the generational indices in the quark Yukawa coupling terms. When expanded out, equation 1.96 produces all of the mass, kinetic, and interaction terms in the Standard Model. The interaction terms include the standard electromagnetic, weak, and strong currents. As well, there are trilinear and quadrilinear interaction terms between gauge bosons associated with non-Abelian groups; the $W^{ \pm}, Z$, and photon couple to each other, as do the eight gluons. Finally, from the covariant derivative of the Higgs field, there are interactions between the Higgs and the electroweak gauge bosons. Note that since the $h$ field remaining after electroweak symmetry breaking is electrically neutral, it does not couple directly to the photon.

\subsubsection{Calculating Amplitudes}

To calculate quantum mechanical amplitudes from a Lagrangian, Feynman rules are typically extracted, from which the amplitude for any perturbative process may be computed to the desired order. Here, we describe the procedure for obtaining the Feynman rules from the Lagrangian without justification; a complete treatment may be found in many quantum field theory textbooks [27, 28, 29, 30].

As always, the Feynman rules for external scalars are trivial; a factor of 1 is introduced into the amplitude for each incoming and outgoing scalar. Incoming fermions give a factor $\psi$, while outgoing fermions give $\bar{\psi}$. Finally, denoting the polarization vector of a spin 1 (vector) boson by $\epsilon_{\mu}$, incoming vector bosons are associated with the factor $\epsilon_{\mu}$, and outgoing vector bosons with $\epsilon_{\mu}^{*}$.

To obtain the Feynman rule for an interaction term in the Lagrangian, one simply omits all of the particle wave functions and multiplies by $i$. For every group of $n$ identical particles in the Lagrangian term, a factor of $n$ ! must be included in the resulting Feynman rule. For example, consider the electron-photon coupling, which 
arises from the electromagnetic current term

$$
\mathcal{L} \supset-e\left(\bar{e} \gamma^{\mu} A_{\mu} e\right)
$$

which may be obtained by expanding the Standard Model Lagrangian, combining the $e_{L}$ and $e_{R}$ terms, and expressing the neutral current in terms of $A_{\mu}$ and $Z_{\mu}$ instead of $B_{\mu}$ and $W_{\mu}^{3}$. This term describes the simple emission or absorption of a photon by an electron, since there is an incoming electron, a photon, and then an outgoing electron. Since there are no identical particles (incoming electrons are distinct from outgoing electrons), the above procedure yields a factor of $-i e \gamma^{\mu}$ for the electron-photon vertex.

Finally, propagators (the Feynman rules for internal particles) are the Green's functions of the equations of motion. They may be obtained by inverting the operator that acts on a field in its equation of motion, and then multiplying by $i$. For example, a scalar particle obeys the Klein-Gordon equation, which may be written in momentum space as

$$
\left(p^{\mu} p_{\mu}-m^{2}\right) \phi=0
$$

The scalar propagator is thus $\frac{i}{p^{2}-m^{2}+i \epsilon}$. The $i \epsilon$ term in the denominator comes from the evaluation of the Green's function, and involves choosing a specific prescription for circumnavigating the poles of a contour integral. There are other choices which affect the sign of this term, and our choice leads to the so-called Feynman propagator. In practice, we will omit the $i \epsilon$ term except when it is important, as in the computation of amplitudes involving loops.

To compute the amplitude for a given process, one draws all the Feynman diagrams that contribute, to the desired order (power of the coupling constant involved). For each diagram, one then multiplies the factors coming from the Feynman rules, working backwards along fermion lines, to obtain the associated amplitude. Whenever there is a momentum that cannot be determined from the external momentum 4-vectors, one 
performs the loop integral by introducing a factor $\int \frac{d^{4} k}{(2 \pi)^{4}}$. The sum of the diagram amplitudes is the total amplitude for the process. After an amplitude is calculated, it may be converted into a cross section or decay rate by including the appropriate phase space integrals. The details can be found in particle physics textbooks such as Griffiths [2].

With the basics of field theory, we may calculate amplitudes for many processes, and extract useful results. However, loop amplitudes often contain divergent integrals, and the treatment of these divergences, known as renormalization, has surprising consequences. We now consider these divergences, and explore the results of renormalization on our ability to describe physics beyond the Standard Model. 


\section{References}

[1] W.-M. Yao et al. Review of particle physics. J. Phys., G33:1-1232, 2006.

[2] David J. Griffiths. Introduction to elementary particles. New York, USA: Wiley (1987) $392 \mathrm{p}$.

[3] C. Quigg. Gauge theories of the strong, weak and electromagnetic interactions. Front. Phys., 56:1-334, 1983.

[4] T. P. Cheng and L. F. Li. Gauge theory of elementary particle physics. Oxford, UK: Clarendon (1984) 536 p. (Oxford Science Publications).

[5] D. Bailin and A. Love. Introduction to gauge field theory. Bristol, UK: Hilger (1986) 348 p. (Graduate Student Series In Physics).

[6] I. J. R. Aitchison and A. J. G. Hey. Gauge theories in particle physics: A practical introduction. Vol. 1: From relativistic quantum mechanics to QED. Bristol, UK: IOP (2003) $406 \mathrm{p}$.

[7] I. J. R. Aitchison and A. J. G. Hey. Gauge theories in particle physics: A practical introduction. Vol. 2: Non-Abelian gauge theories: QCD and the electroweak theory. Bristol, UK: IOP (2004) $454 \mathrm{p}$.

[8] D. H. Perkins. Introduction to high-energy physics. Reading, USA: AddisonWesley (1982) $437 \mathrm{p}$.

[9] Pierre Ramond. Journeys beyond the Standard Model. Reading, Mass., Perseus Books, 1999.

[10] Gerard 't Hooft. 1972. Marseille Conference (unpublished).

[11] Gerard 't Hooft. An algorithm for the poles at dimension four in the dimensional regularization procedure. Nucl. Phys., B62:444-460, 1973. 
[12] D. J. Gross and Frank Wilczek. Asymptotically free gauge theories. 1. Phys. Rev., D8:3633-3652, 1973.

[13] D. J. Gross and Frank Wilczek. Ultraviolet behavior of non-Abelian gauge theories. Phys. Rev. Lett., 30:1343-1346, 1973.

[14] H. David Politzer. Reliable perturbative results for strong interactions? Phys. Rev. Lett., 30:1346-1349, 1973.

[15] Yoichiro Nambu. Quasi-particles and gauge invariance in the theory of superconductivity. Phys. Rev., 117:648-663, 1960.

[16] Peter W. Higgs. Broken symmetries, massless particles and gauge fields. Phys. Lett., 12:132-133, 1964.

[17] F. Englert and R. Brout. Broken symmetry and the mass of gauge vector mesons. Phys. Rev. Lett., 13:321-322, 1964.

[18] Peter W. Higgs. Broken symmetries and the masses of gauge bosons. Phys. Rev. Lett., 13:508-509, 1964.

[19] G. S. Guralnik, C. R. Hagen, and T. W. B. Kibble. Global conservation laws and massless particles. Phys. Rev. Lett., 13:585-587, 1964.

[20] Peter W. Higgs. Spontaneous symmetry breakdown without massless bosons. Phys. Rev., 145:1156-1163, 1966.

[21] T. W. B. Kibble. Symmetry breaking in non-Abelian gauge theories. Phys. Rev., 155:1554-1561, 1967.

[22] S. L. Glashow. Partial symmetries of weak interactions. Nucl. Phys., 22:579-588, 1961.

[23] Abdus Salam and John Clive Ward. Electromagnetic and weak interactions. Phys. Lett., 13:168-171, 1964.

[24] Steven Weinberg. A model of leptons. Phys. Rev. Lett., 19:1264-1266, 1967.

[25] K. Nishijima. Charge independence theory of V particles. Progress of Theoretical Physics, 13:285-304, March 1955.

[26] Murray Gell-Mann. Nuovo Cim., Suppl. 4:848, 1956.

[27] Michael Edward Peskin and Daniel V. Schroeder. An introduction to quantum field theory. Reading, USA: Addison-Wesley (1995) 842 p. 
[28] A. Zee. Quantum field theory in a nutshell. Princeton, USA: Princeton Univ. Pr. (2003) 518 p.

[29] L. H. Ryder. Quantum field theory. Cambridge, UK: Univ. Pr. (1985) 443 p.

[30] Pierre Ramond. Field theory: a modern primer. Front. Phys., 74:1-329, 1989. 


\title{
Chapter 2
}

\section{Effective Field Theory}

\begin{abstract}
Although the Standard Model has correctly made many experimental predictions, it is believed that new physics exists at higher energy scales. We will consider how the parameters of the Standard Model vary with energy, as they are "renormalized" by the integration over the high energy modes of the fields $[1,2]$. We then introduce effective field theories, which can provide a model-independent way of describing the low-energy effects of new physics $[3,4,5]$. As an example, we will consider the successful renormalization of interactions mediated by the weak force, as we integrate massive fields out of the Standard Model to construct a low energy effective theory representation. We shall see that renormalization is made significantly easier by effective field theories.
\end{abstract}

\subsection{Renormalization}

Consider the Feynman diagram shown in figure 2.1. This diagram is a correction to the scalar propagator in $\phi^{4}$ theory. Since the propagator for a scalar particle depends on its mass, the diagram corresponds to an adjustment of the $\phi$ mass. To see this in a more quantitative manner, we denote the sum of all one-particle irreducible (1PI) corrections to the $\phi$ propagator by $-i M^{2}\left(p^{2}\right)$. (An $n$-particle irreducible diagram 


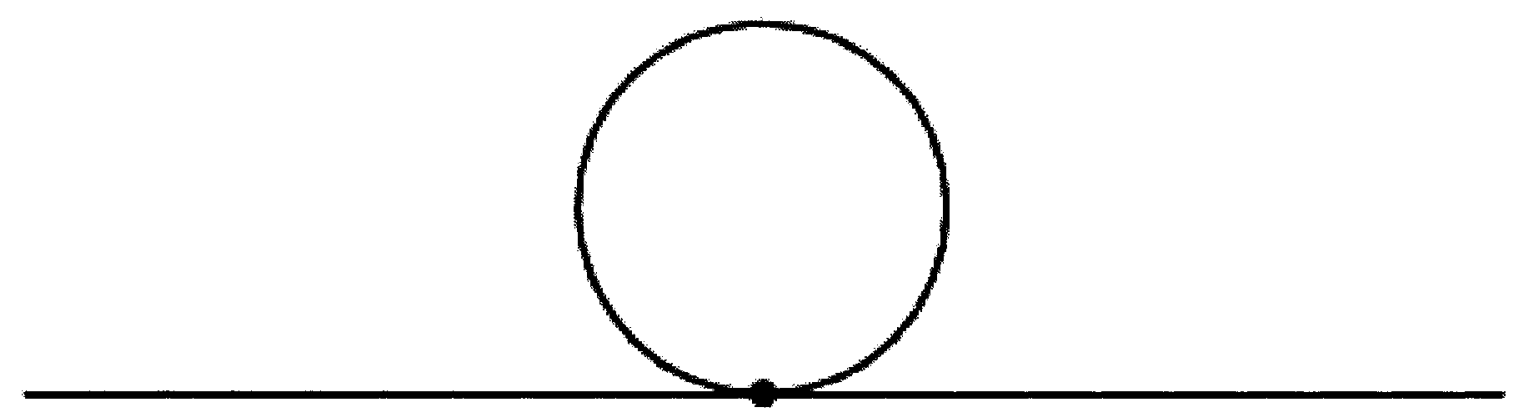

Figure 2.1: The lowest order correction to the scalar propagator of $\phi^{4}$ theory.

is one that cannot be broken up, or "reduced", into two separate diagrams by the removal of $n$ particle propagators.) Note that all corrections to the propagator that are 2PI, but not $1 \mathrm{PI}$, may be formed by two successive insertions of $-i M^{2}\left(p^{2}\right)$, with the bare $\phi$ propagator in between them. In this fashion, the full $\phi$ propagator may be systematically constructed with an infinite series:

$$
\begin{aligned}
\text { Full propagator } & =\frac{i}{p^{2}-m^{2}}+\frac{i}{p^{2}-m^{2}}\left(-i M^{2}\left(p^{2}\right)\right) \frac{i}{p^{2}-m^{2}}+\ldots \\
& =\frac{i}{p^{2}-m^{2}}\left(1-\frac{M^{2}\left(p^{2}\right)}{p^{2}-m^{2}}\right)^{-1} \\
& =\frac{i}{p^{2}-m^{2}-M^{2}\left(p^{2}\right)}
\end{aligned}
$$

The amplitude for the diagram in figure 2.1 is given by

$$
\mathcal{M}=\frac{\lambda}{2} \int \frac{d^{4} k}{(2 \pi)^{4}} \frac{1}{k^{2}-m^{2}}
$$

where since two of the four particles meeting at the $\phi^{4}$ vertex come from the same propagator, we have introduced a symmetry factor of $\frac{1}{2}$. There are four powers of the loop momentum $k$ in the numerator of the integral and only two powers of $k$ in the denominator, so the amplitude is quadratically divergent. If we were to cut 
off the integral at a mass scale $\Lambda$, the integral would go as $\Lambda^{2}$. We will look at a mass-independent way to regulate this integral, known as dimensional regularization.

\subsubsection{Dimensional Regularization}

The lowest-order correction to the $\phi$ propagator, like many loop diagrams in quantum field theory, is divergent (infinite). While there are different procedures to regulate divergences, the most commonly used mass-independent prescription is dimensional regularization. Dimensional regularization is performed by computing a process in $d=4-\epsilon$ dimensions, and then taking the limit as $\epsilon \rightarrow 0$.

In $d$ dimensions, the action is $S=\int d^{d} x \mathcal{L}$ (in $\hbar=c=1$ units). For the action to be dimensionless, then, $\mathcal{L}$ must have mass dimension $d$. We choose to leave the free terms, proportional to $\left(\partial_{\mu} \phi\right)\left(\partial^{\mu} \phi\right)$ and $m^{2} \phi^{2}$, unaltered. This choice corresponds to giving mass dimension $\frac{1}{2}(d-2)$ to the field $\phi$. Consequently, the interaction term must be modified to $-\frac{\lambda}{4 !} \mu^{4-d} \phi^{4}$, where $\mu$ is a parameter with mass dimension 1 . The $\phi$ propagator correction thus becomes

$$
\mathcal{M}=\frac{\lambda}{2} \mu^{4-d} \int \frac{d^{d} k}{(2 \pi)^{d}} \frac{1}{k^{2}-m^{2}}
$$

The evaluation of integrals arising in dimensional regularization is covered in Peskin and Schroeder [6], and some of the results are listed in appendix A. The result for the amplitude,

$$
\mathcal{M}=-i\left(\frac{\lambda}{2}\right) \frac{m^{2}}{(4 \pi)^{2}} \Gamma\left(1-\frac{d}{2}\right)\left(\frac{4 \pi \mu^{2}}{m^{2}}\right)^{2-\frac{d}{2}}
$$

contains a pole as $d \rightarrow 4$, since the $\Gamma$ function has a pole at -1 . At one loop, this is the only correction to the $\phi$ propagator, so we identify $\mathcal{M}$ with $-i M^{2}\left(p^{2}\right)$. We see that the correction corresponds to an infinite adjustment of the $\phi$ mass, as expected, because $\mathcal{M}$ contained a divergent integral. 


\subsubsection{Counterterms}

At the expense of introducing extra Feynman rules, we can rewrite $\mathcal{L}$ to prevent infinite quantities from appearing in physical results. We first replace $m$ by $m_{0}$ in the Lagrangian, to indicate that the mass term only gives the bare mass:

$$
\mathcal{L}=\frac{1}{2}\left(\partial^{\mu} \phi\right)\left(\partial_{\mu} \phi\right)-\frac{1}{2} m_{0}^{2} \phi^{2}-\frac{\lambda}{4 !} \phi^{4}
$$

Now, writing the physically observed mass as $m$, we split the mass term into two pieces, yielding

$$
\mathcal{L}=\frac{1}{2}\left(\partial^{\mu} \phi\right)\left(\partial_{\mu} \phi\right)-\frac{1}{2} m^{2} \phi^{2}-\frac{1}{2} \delta_{m} \phi^{2}-\frac{\lambda}{4 !} \phi^{4}
$$

where we have made the definition

$$
\delta_{m}=m_{0}^{2}-m^{2}
$$

The $\delta_{m}$ term is called a counterterm, and contains the infinite adjustment to the $\phi$ mass computed above. The Lagrangian now describes a scalar field of mass $m$ (the physical mass, not the bare mass as before) with two interactions. One is the standard quartic interaction, but the other has strength $\delta_{m}$ and couples two scalars together. Whenever one of the diagrams for a process involves a bare $\phi$ propagator, it may be separated into two diagrams, one involving the physical $\phi$ propagator and the other having the mass counterterm instead.

Masses are not the only parameters that receive infinite adjustments from loop corrections. In general, counterterms are also needed to renormalize couplings. In $\phi^{4}$ theory, we absorb corrections to the $\phi^{4}$ vertex into the quantity $\delta_{\lambda}$. We will not compute $\delta_{\lambda}$ explicitly here, but it may be determined in the same manner as $\delta_{m}$.

If we now consider two-loop diagrams as well, we will obtain more divergent corrections to the $\phi$ propagator. However, these corrections require us to refine our 
renormalization procedure. Note that the one-loop correction to the $\phi$ propagator was independent of the external momentum $p$. In general, this is not the case, and we must choose an energy scale at which to perform renormalization. Conventionally, counterterms are added such that the pole in the $\phi$ propagator is $\frac{i}{p^{2}-m^{2}}$ at $p^{2}=m^{2}$. (Note that a similar condition must be specified for each renormalized parameter. In massive $\phi^{4}$ theory, the conventional choice is to require that the sum of the vertex and the vertex counterterm yields $-i \lambda$ at $s=4 m^{2}, t=u=0$.) This condition fixes not only the location of the pole at $p^{2}=m^{2}$, but also determines its residue to be 1 . The location of the pole may be changed by adjusting $\delta_{m}$, but the residue requires a different procedure, known as field strength renormalization.

Certain corrections to propagators, which only appear at two loops in $\phi^{4}$ theory, have the effect of changing the propagator to $\frac{i Z}{p^{2}-m^{2}}$, i.e., change the residue of the propagator pole. To satisfy the renormalization condition, we write $\mathcal{L}$ in terms of the rescaled field

$$
\phi_{r}=Z^{-\frac{1}{2}} \phi_{0}
$$

where $\phi_{0}$ is the original (bare) scalar field, and $Z$ is called the field strength renormalization of $\phi$. In the above expression, the exponent of $Z$ is chosen such that the $\phi_{r}$ propagator, which is proportional to $\phi_{r}(x) \phi_{r}(y)$, has no $Z$ dependence. In terms of the rescaled field $\phi_{r}$, the Lagrangian for $\phi^{4}$ theory is

$$
\mathcal{L}=\frac{1}{2} Z\left(\partial^{\mu} \phi_{r}\right)\left(\partial_{\mu} \phi_{r}\right)-\frac{1}{2} Z m_{0}^{2} \phi^{2}-Z^{2} \frac{\lambda_{0}}{4 !} \phi^{4},
$$

where we have denoted the bare mass and coupling constant by $m_{0}$ and $\lambda_{0}$, respectively. Now that we have accounted for the adjustments of all the parameters in the theory, we again write $\mathcal{L}$ as the sum of the canonical Lagrangian terms for a scalar 
theory and the associated counterterms. Defining

$$
\begin{aligned}
& \delta_{Z}=Z-1 \\
& \delta_{m}=Z m_{0}^{2}-m^{2} \\
& \delta_{\lambda}=Z^{2} \lambda_{0}-\lambda
\end{aligned}
$$

we have the Lagrangian

$$
\begin{aligned}
\mathcal{L} & =\frac{1}{2}\left(\partial^{\mu} \phi_{r}\right)\left(\partial_{\mu} \phi_{r}\right)-\frac{1}{2} m^{2} \phi_{r}^{2}-\frac{\lambda}{4 !} \phi_{r}^{4} \\
& +\frac{1}{2} \delta_{Z}\left(\partial^{\mu} \phi_{r}\right)\left(\partial_{\mu} \phi_{r}\right)-\frac{1}{2} \delta_{m} \phi_{r}^{2}-\frac{\delta_{\lambda}}{4 !} \phi_{r}^{4}
\end{aligned}
$$

The counterterms lead to new Feynman rules. Each bare propagator $\frac{i}{p^{2}-m_{0}^{2}}$ in a diagram is effectively split into two pieces, the physical propagator $\frac{i}{p^{2}-m^{2}}$ and the counterterm $i\left(p^{2} \delta_{Z}-\delta_{m}\right)$. Similarly, each bare quartic vertex can be separated into the physical vertex and the vertex counterterm. We may compute the quantities $\delta_{Z}, \delta_{m}$, and $\delta_{\lambda}$ to any number of loops, although higher-order corrections require calculation of exponentially more diagrams. With counterterms, the calculation of any physical process will yield finite results.

\subsubsection{The Hierarchy Problem}

In the Standard Model, the Higgs propagator receives corrections from loops involving both fermions and bosons. We have seen that these corrections correspond to adjustments of the Higgs mass, and are quadratically divergent. Let us try to regulate these divergences by cutting off the loop momentum at the scale $\Lambda$. Then the corrections to the Higgs mass will be finite, and will go as $\Lambda^{2}$. It makes sense to interpret $\Lambda$ as the scale at which new physics appears, since it is at this point that our theory breaks down. Suppose that no new physics appears until the scale of gravity, which 
is $G_{N}^{-\frac{1}{2}}$ in $\hbar=c=1$ units, where $G_{N}$ is Newton's constant. Then we would have $\Lambda \sim 10^{19} \mathrm{GeV}$.

However, precision electroweak data from colliders show that the Higgs mass must be $\mathcal{O}(100 \mathrm{GeV})$ [7]. Although fermionic and bosonic corrections to the Higgs mass have opposite sign, an extremely precise cancellation (to a few dozen significant figures) would have to take place between them to yield a mass shift no larger than the predicted mass. There is no mechanism in the Standard Model for generating such a cancellation. This issue is known as the hierarchy problem, and is one of the reasons that new physics is expected to lie just beyond the electroweak scale.

There are many different models of new physics, and they deal with the hierarchy problem in various ways. For instance, supersymmetry introduces a symmetry between fermions and bosons, leading to the desired cancellation of corrections to the Higgs mass. In section 3.3, we will consider some extensions of the Standard Model, and place limits on them using experimental data.

\subsubsection{The Callan-Symanzik Equation}

We have seen that in general, quantum corrections to a field theory depend on the renormalization scale. However, if we write the Lagrangian in terms of the bare parameters $\phi_{0}, m_{0}$, and $\lambda_{0}$, then all of its terms are independent of the scale chosen for renormalization. Consequently, the bare Green's functions, which give the amplitude for particles to propagate between points in spacetime, are invariant under changes in the renormalization scale.

For simplicity, we consider massless $\phi^{4}$ theory. Since massive $\phi^{4}$ theory included counterterms that were inversely proportional to powers of $\mathrm{m}^{2}$, we must modify the renormalization conditions to avoid counterterm singularities. We now require that for spacelike momenta satisfying $p^{2}=-M^{2}$, the sum of all 1PI contributions to the 
$\phi$ propagator must be zero and have zero derivative with respect to $p^{2}$. Similarly, we require that the $\phi^{4}$ vertex have coupling constant $-i \lambda$ at $s=t=u=-M^{2}$.

Suppose we have a Green's function $G^{(n)}$ involving $n$ rescaled scalar fields, where the field strength renormalization is $Z$. Then since each rescaled $\phi$ only differs from $\phi_{0}$ by a factor of $Z^{-\frac{1}{2}}, G^{(n)}$ must be related to the bare Green's function $G_{0}^{(n)}$ by

$$
G^{(n)}=Z^{-\frac{n}{2}} G_{0}^{(n)}
$$

Under an infinitesimal shift of the renormalization scale

$$
M \rightarrow M+\delta M,
$$

there must be corresponding shifts in $\lambda$ and $Z$ to keep the bare Green's functions unchanged:

$$
\begin{aligned}
& \lambda \rightarrow \lambda+\delta \lambda, \\
& Z \rightarrow Z+\delta Z .
\end{aligned}
$$

Since the bare Green's function $G_{0}^{(n)}=Z^{\frac{n}{2}} G^{(n)}$ must remain unchanged, we have

$$
\begin{aligned}
& 0=(Z+\delta Z)^{\frac{n}{2}}\left(G^{(n)}+\delta G^{(n)}\right)-Z^{\frac{n}{2}} G^{(n)} \\
& 0=\left(\frac{n}{2} \frac{\delta Z}{Z}+\frac{\delta G^{(n)}}{G^{(n)}}\right) G^{(n)}
\end{aligned}
$$

Defining the anomalous dimension

$$
\gamma=\frac{1}{2} M \frac{\delta(\log Z)}{\delta M}
$$


we may write the above as

$$
0=\left(n \gamma \frac{\delta M}{M}+\frac{\delta G^{(n)}}{G^{(n)}}\right) G^{(n)}
$$

Now, considering the renormalized Green's function $G^{(n)}$ as a function of $M$ and $\lambda$, we may also write

$$
\delta G^{(n)}=\frac{\partial G^{(n)}}{\partial \lambda} \delta \lambda+\frac{\partial G^{(n)}}{\partial M} \delta M
$$

and if we take

$$
\beta=M \frac{\delta \lambda}{\delta M}
$$

then the change in the renormalized Green's function becomes

$$
\delta G^{(n)}=\frac{\delta M}{M}\left(M \frac{\partial}{\partial M}+\beta \frac{\partial}{\partial \lambda}\right) G^{(n)}
$$

Putting equations 2.20 and 2.23 together, we have

$$
\left(M \frac{\partial}{\partial M}+\beta \frac{\partial}{\partial \lambda}+n \gamma\right) G^{(n)}=0
$$

which is the Callan-Symanzik equation $[8,9]$. Note that $\beta$ and $\gamma$ are dimensionless, and since the only scale in $G^{(n)}$ is the renormalization scale $M$, they depend solely on $\lambda$ by dimensional analysis [6]. In particular, since $M$ is the energy scale at which we are defining our theory, the $\beta$ function provides a measure of how the coupling changes with energy, and a nonzero value leads to a running coupling constant. If we now let $M_{0}$ be some reference renormalization point and consider the coupling constant as a function of the energy scale $p$, we have

$$
\frac{d}{d \log \left(p / M_{0}\right)} \lambda(p)=\frac{p}{M_{0}} \frac{d \lambda(p)}{d\left(p / M_{0}\right)}=p \frac{d \lambda(p)}{d p}=\beta
$$


where we have taken the energy scale $p$ to be our renormalization scale. This is the renormalization group equation for $\lambda$, and describes the evolution of the coupling with energy. The initial condition for the solution of this equation is provided by the renormalization condition for $\lambda$, which gives the coupling constant some specific value at $p=M_{0}$.

Now, after we separate our Lagrangian into terms involving physical quantities and counterterms, the only dependence on the renormalization scale is in the latter. We may thus calculate $\beta$ and $\gamma$ by isolating the contributions of the counterterms to the appropriate Green's functions, and then applying the Callan-Symanzik equation.

As a trivial example, consider the one-loop computation of the anomalous dimension in massless $\phi^{4}$ theory. We have already seen that at lowest order in $\phi^{4}$ theory, there is no correction to the field strength renormalization, i.e. $\delta_{Z}=0$. This expression is clearly independent of $M$, so we have $\gamma=0$. At higher order, we would apply the Callan-Symanzik equation to the $\phi$ propagator, which is $G^{(2)}$, to find $\gamma$.

In $\mathrm{QED}$ and $\mathrm{QCD}$, the $\beta$ function goes as the cube of the coupling, and we may write

$$
\frac{d}{d \log \left(p / M_{0}\right)} g=-\frac{b_{0}}{(4 \pi)^{2}} g^{3}
$$

at lowest order. (There are extra terms involving $b_{1}$, etc. in higher order corrections.) In terms of the associated constant

$$
\alpha=\frac{g^{2}}{4 \pi}
$$

this renormalization group equation may be written as

$$
\frac{d}{d \log \left(p / M_{0}\right)} \alpha=-\frac{b_{0}}{2 \pi} \alpha^{2}
$$


If our renormalization condition requires $\alpha=\alpha^{*}$ at $p=M_{0}$, then the solution is

$$
\alpha(p)=\frac{\alpha^{*}}{1+\alpha^{*} \frac{b_{0}}{2 \pi} \log \left(p / M_{0}\right)}
$$

Note that this expression has a pole at the scale

$$
\Lambda=M_{0} e^{-\frac{2 \pi}{b_{0} \alpha^{*}}}
$$

and by rewriting our expression for $\alpha$ in terms of $\Lambda$ [6], we may simplify it to

$$
\alpha(p)=\frac{2 \pi}{b_{0} \log (p / \Lambda)}
$$

If $b_{0}$ is positive, as in QCD, then $\alpha$ decreases for increasing $p$. Since such theories interact very weakly at high energies, they are called asymptotically free.

\subsubsection{Local Operators}

The above discussion may be generalized to local operators. Defining the renormalization of an operator $\mathcal{O}$ to be $Z_{\mathcal{O}}$, we have

$$
\mathcal{O}=Z_{\mathcal{O}} \mathcal{O}_{0}
$$

where $\mathcal{O}_{0}$ is the operator written in terms of bare fields. For instance, for the mass

operator $\mathcal{O}=\phi^{2}$, we have $\mathcal{O}_{0}=\phi_{0}^{2}$. If $\mathcal{O}$ involves $n$ fields, we may construct a Green's function from the vacuum expectation value of $\phi\left(p_{1}\right) \cdots \phi\left(p_{n}\right) \mathcal{O}(k)$. Then, we apply the same analysis to this Green's function as before. Requiring that the bare version of this Green's function remains unchanged under shifts in the renormalization scale $M$, we get

$$
\left(M \frac{\partial}{\partial M}+\beta \frac{\partial}{\partial \lambda}+n \gamma+\gamma_{\mathcal{O}}\right) G^{(n)}=0
$$


where the anomalous dimension of $\mathcal{O}$ is given by

$$
\gamma_{\mathcal{O}}=M \frac{\partial}{\partial M} \log Z_{\mathcal{O}}(M)
$$

Note that our result extends in a straightforward manner to theories with more than one field; there is a different anomalous dimension for each field, and we must keep track of them separately.

In practice, $\gamma_{\mathcal{O}}$ is computed from the counterterms of a theory, since these are the only terms in $\mathcal{L}$ with any dependence on $M$. For example, consider the QCD renormalization of an operator $\mathcal{O}$ containing $n$ quark fields and $k$ gluon fields. After choosing a renormalization condition, the counterterm $\delta_{\mathcal{O}}$ may be computed in terms of the renormalization scale $M$. Then, the anomalous dimension of $\mathcal{O}$ is given by

$$
\gamma_{\mathcal{O}}=M \frac{\partial}{\partial M}\left(-\delta_{\mathcal{O}}+\frac{n}{2} \delta_{2}+\frac{k}{2} \delta_{3}\right)
$$

where $\delta_{2}$ and $\delta_{3}$ are the counterterms associated with the field strength renormalization of the quark and gluon, respectively, and are analogous to $\delta_{Z}$ in $\phi^{4}$ theory. While we omit the details of the proof of this statement (see [6] for details), it is clear that the result makes sense qualitatively. The anomalous dimension of $\mathcal{O}$ is given by the $M$ dependence of $Z_{\mathcal{O}}$. Since $Z_{\mathcal{O}}$ is the rescaling factor between the bare operator $\mathcal{O}_{0}$ and the rescaled operator $\mathcal{O}$, its $M$ dependence comes from the renormalization of the fields in $\mathcal{O}$, as well as any counterterm needed so that $\mathcal{O}$ satisfies its renormalization condition. We thus expect $\gamma_{\mathcal{O}}$ to depend on $\delta_{2}, \delta_{3}$, and $\delta_{\mathcal{O}}$.

Finally, just as the $\beta$ function led to a renormalization group equation for the coupling constant, the anomalous dimension of an operator yields a differential equation for the evolution of its coefficient. If the operator $\mathcal{O}$ has anomalous dimension $\gamma_{\mathcal{O}}$ 
and coefficient $c$, then we have the renormalization group equation

$$
\frac{d}{d \log \left(p / M_{0}\right)} c(p)=\gamma_{\mathcal{O}} c(p)
$$

where the initial condition is again given by the renormalization condition, and is of the form

$$
c\left(M_{0}\right)=c^{*}
$$

In general, the anomalous dimension $\gamma_{\mathcal{O}}$ will involve the coupling constants of the theory, and so we must substitute the solutions of the renormalization group equations for the couplings. For instance, anomalous dimensions of operators can often be written as

$$
\gamma_{\mathcal{O}}=-a_{\mathcal{O}} \frac{g^{2}}{(4 \pi)^{2}}
$$

where $g$ is the coupling in the theory and $a_{\mathcal{O}}$ does not depend on $g$. In this case, using the definition of $\Lambda$ in equation 2.30 and the renormalization condition, we have the solution

$$
c(p)=\left(\frac{\log (p / \Lambda)}{\log \left(M_{0} / \Lambda\right)}\right)^{-\frac{a_{\mathcal{O}}}{2 b_{0}}} c^{*}
$$

where $b_{0}$ parametrizes the $\beta$ function for the coupling $g$, as in the previous section. This result will be useful for computing QCD corrections. After choosing the renormalization condition for a given operator and computing its anomalous dimension, we may use the QCD $\beta$ function, given by

$$
b_{0}=11-\frac{2}{3} n_{f}
$$

where $n_{f}$ is the number of flavours, to obtain the QCD running of the operator. 


\subsection{Example: Fermi Theory Of Weak Interactions}

The charged weak interaction couples the left-handed components of the up-type $(u$, $c, t)$ quarks to those of the down-type quarks $(d, s, b)$, with the mixing parameters specified by the CKM matrix. The Feynman rule associated with this interaction is

$$
-\frac{i g}{\sqrt{2}} V^{i j} \bar{u}_{L}^{i} \gamma^{\mu} d_{L}^{j}+\text { h.c. }
$$

where $i$ and $j$ are generational indices, $g$ is the coupling strength of the weak interaction, and the factor of $\sqrt{2}$ comes from the normalization of the $W^{ \pm}$fields.

At low energies, the mediating $W$ bosons may only exist as virtual particles. Now, for energy scales much lower than $m_{W} \sim 80 \mathrm{GeV}$, the $W$ propagator may be approximated by

$$
-\frac{i\left(g_{\mu \nu}-\frac{p_{\mu} p_{\nu}}{m_{W}^{2}}\right)}{p^{2}-m_{W}^{2}} \approx \frac{i g_{\mu \nu}}{m_{W}^{2}} .
$$

Now consider the process $s \bar{u} \rightarrow d \bar{u}$. At tree level, this process is mediated by a $W$ boson. Neglecting QCD corrections, we may approximate the amplitude at low energies by

$$
\mathcal{M}=-i \frac{g^{2}}{2 m_{W}^{2}} V^{u s} V^{u d *}\left(\bar{u}_{L} \gamma^{\mu} s_{L}\right)\left(\bar{d}_{L} \gamma_{\mu} u_{L}\right)
$$

Note that this approximation to the amplitude may be obtained immediately by writing down the Feynman rule associated with the effective Lagrangian

$$
\mathcal{L} \supset-\frac{g^{2}}{2 m_{W}^{2}} V^{u s} V^{u d *}\left(\bar{u}_{L} \gamma^{\mu} s_{L}\right)\left(\bar{d}_{L} \gamma_{\mu} u_{L}\right)
$$

Fermi's original theory of the weak interaction [10] involved 4-fermion vertices similar to the one we have constructed in our effective Lagrangian.

The general idea of effective field theory is to integrate out the effects of high energy processes, leaving an effective action that can be used to perform low energy 


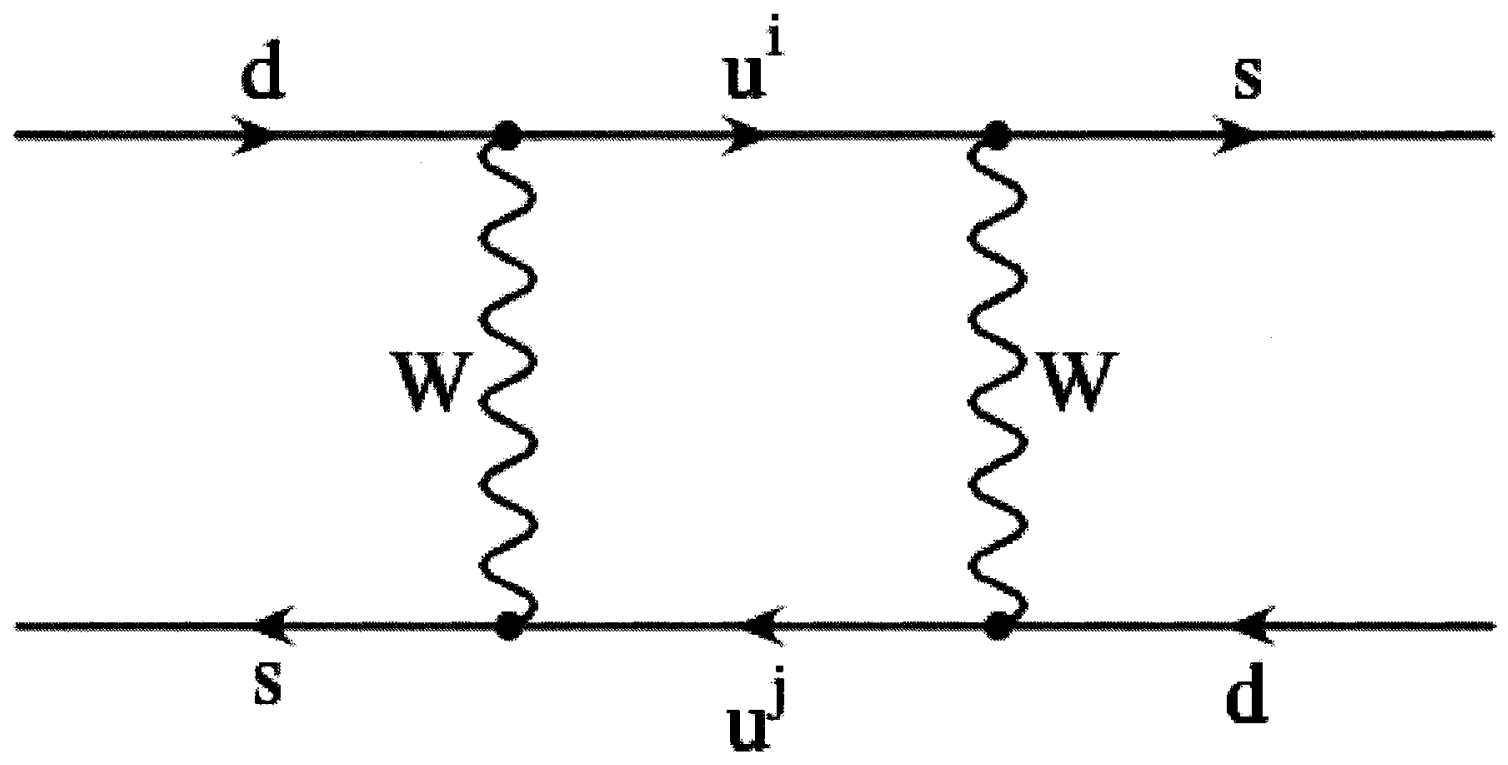

Figure 2.2: $K-\bar{K}$ mixing in the Standard Model.

computations [1]. We will see that effective field theories are particularly useful in considering the effects of renormalization.

\subsection{Example: Effective Field Theory Of $\Delta S=2$ Interactions In The Standard Model}

If we assign the $s$ quark a strangeness of -1 and its antiparticle a strangeness of +1 , it is clear that $W$ exchange may violate strangeness by, at most, one unit. Processes that violate strangeness by two units occur, at lowest order, through box diagrams. We will develop an effective field theory to compute the amplitude for such a process, $K-\bar{K}$ mixing [5], shown in figure 2.2. Throughout the calculation, we neglect the mass of the $u$ quark. Also, we assume that the scale of the weak interactions, $m_{W}$, is much larger than the masses of any of the quarks. In practice, the latter assumption 


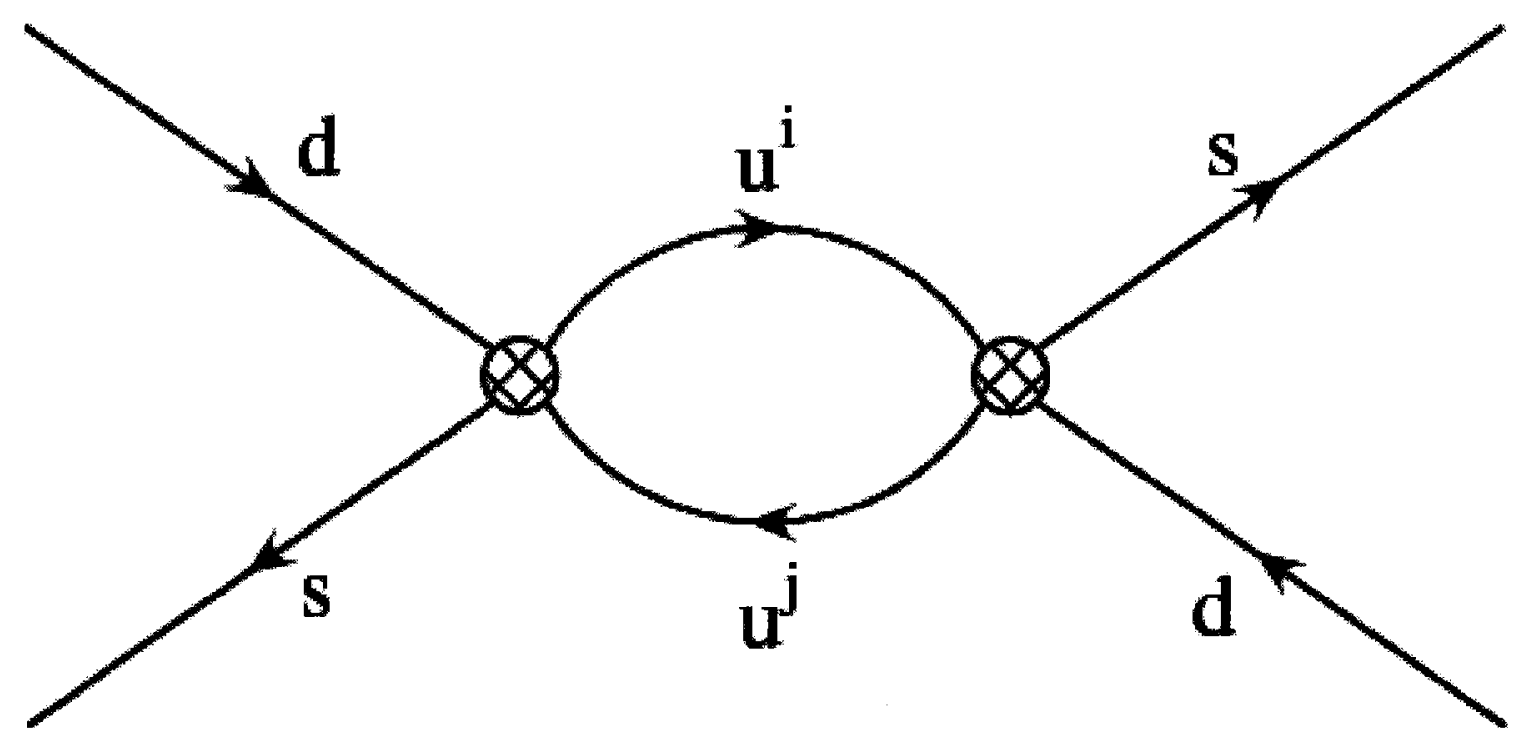

Figure 2.3: The contribution of the $\Delta S=1$ operator to $K-\bar{K}$ mixing.

is false, as the top quark has a mass of about $173 \mathrm{GeV} \sim 2 m_{W}$. However, this is the approach in the original literature $[11,12]$, and so we follow the original treatment as an illustration of the application of effective field theory techniques.

\subsubsection{The GIM Mechanism}

In the effective theory, there are two types of contributions to $K-\bar{K}$ mixing. The first, shown in figure 2.3 , is simply the result of two insertions of the $\Delta S=1$ operator from the Fermi theory of the weak interaction. The Lagrangian term associated with this vertex is

$$
-\frac{g^{2}}{2 m_{W}^{2}} V^{i d} V^{j s *}\left(\bar{u}_{L}^{i} \gamma^{\mu} d_{L}\right)\left(\bar{s}_{L} \gamma_{\mu} u_{L}^{j}\right)=-\frac{4 G_{F}}{\sqrt{2}} V^{i d} V^{j s *}\left(\bar{u}_{L}^{i} \gamma^{\mu} d_{L}\right)\left(\bar{s}_{L} \gamma_{\mu} u_{L}^{j}\right)
$$




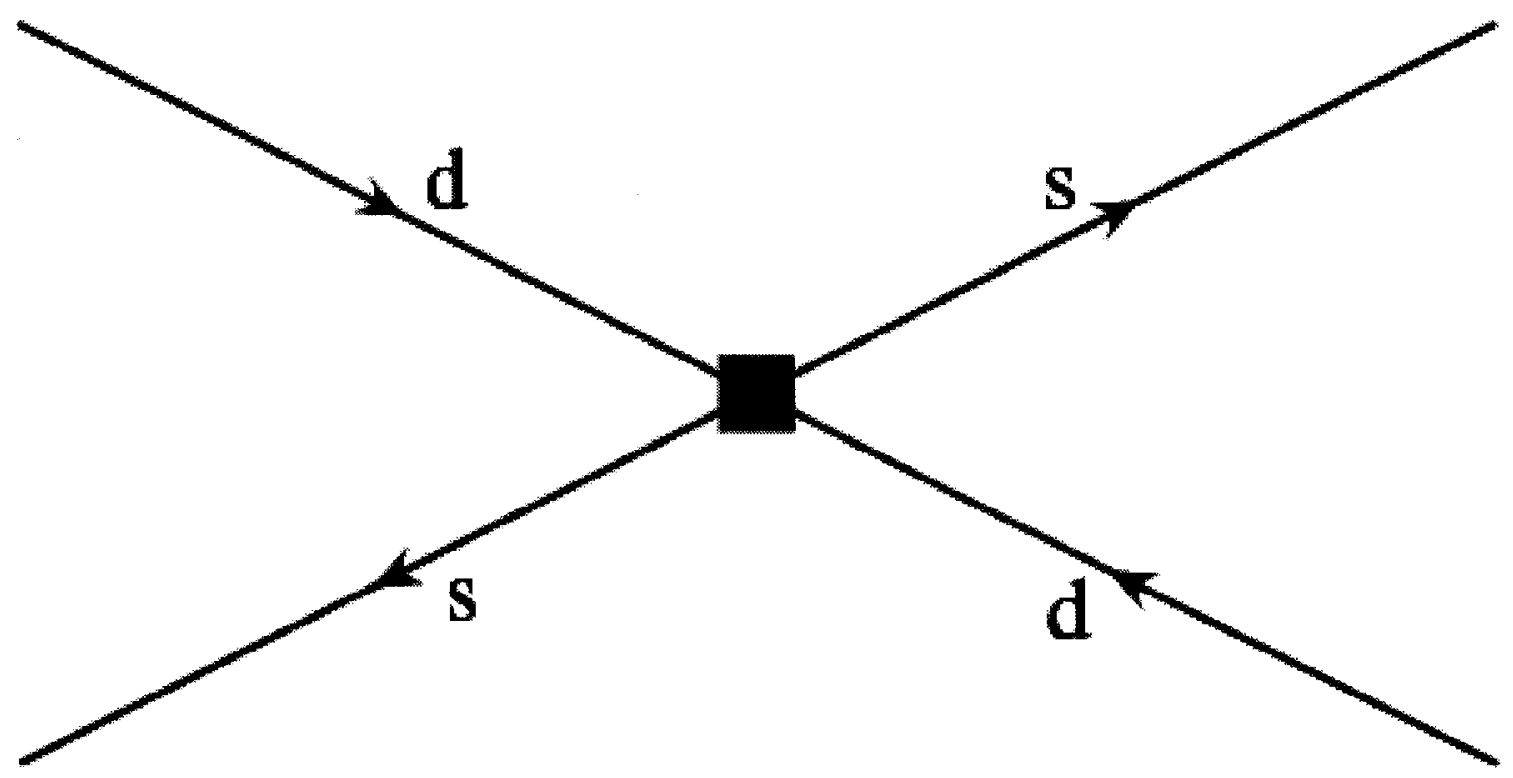

Figure 2.4: The contribution of the $\Delta S=2$ operator to $K-\bar{K}$ mixing.

where $1 \leq i, j \leq 3$ and we have defined the Fermi constant

$$
G_{F}=\frac{1}{4 \sqrt{2}} \frac{g^{2}}{m_{W}^{2}}
$$

If the diagram in figure 2.3 and the diagram in the full theory are both finite, then their amplitudes should be equivalent. However, if either diagram is divergent, we must perform renormalization. Generally, renormalization will affect the diagrams differently. We absorb the difference between the two diagrams, after renormalization, into a local four-fermion operator proportional to $\left(\bar{s}_{L} \gamma^{\mu} d_{L}\right)\left(\bar{s}_{L} \gamma_{\mu} d_{L}\right)$. This operator is shown in figure 2.4 .

By power counting, we may show that in the full theory, the diagram for $K-\bar{K}$ mixing is convergent. Ignoring particle masses, each of the two $W$ propagators comes with a factor of $\frac{1}{k^{2}}$, where $k$ is the loop momentum. Also, each of the two quark propagators comes with a factor of $\frac{1}{k}$. The loop integral thus goes as $\int \frac{d^{4} k}{k^{6}}$. 
However, the effective theory diagram of figure 2.3 appears to be divergent, since the $W$ propagators have been removed. We will now show that the divergent part of the amplitude in figure 2.3 vanishes due to the unitarity of the CKM matrix. The amplitude receives nine contributions, since there are three possible quark flavour choices for each of the two propagators. Suppose that one of the propagators in figure 2.3 has no mass insertions. In this case, the contributions arising from different flavour choices for this propagator will differ only by the CKM matrix elements. Since each propagator couples to a down quark at one end and a strange quark at the other, the total amplitude would be proportional to

$$
V^{u d} V^{u s *}+V^{c d} V^{c s *}+V^{t d} V^{t s *}=0
$$

by unitary of the CKM matrix. So if one of the propagators has no mass insertions, the amplitude for the diagram vanishes. This cancellation is known as the GIM mechanism, after Glashow, Iliopoulos, and Maiani [13].

On the other hand, if both propagators have mass insertions, then each of the nine contributions will be weighted differently, and the cancellation will not take place. Now, note that since the weak interaction only couples to left-handed particles, and mass terms flip chirality (handedness), there must be an even number of mass insertions on each quark propagator. At lowest order, then, there must be two mass insertions on each propagator, dividing each propagator into three segments. Since fermion propagators go as $\frac{1}{k}$, the loop diagram will go as $\int \frac{d^{4} k}{k^{6}}$, and will thus converge.

At energies below $m_{W}$ but above $m_{t}$ in the hypothetical but pedagogically motivated case $m_{t}<m_{W}$, then, the operator in figure 2.4 vanishes. Therefore, $K-\bar{K}$ mixing may be described in terms of a single effective operator that violates strangeness by one unit. Going down in energy, this analysis applies until we reach $m_{t}$, because below this energy the GIM cancellation does not take place. 


\subsubsection{Integrating Out $t$}

Below the $W$ mass, the effective theory does not contain any $W$ propagators. The influence of the $W$ is only felt through couplings involving $G_{F}$, which depend on $m_{W}$. Similarly, below the top quark mass, we must modify the effective theory so that the amplitude for $K-\bar{K}$ mixing can be written solely in terms of local operators and light (non-top) quarks.

The first effect of integrating out the top quark is that our effective $\Delta S=1$ operators, defined in equation 2.45 for energies above $m_{t}$, cannot involve $t$ anymore. The operators that don't involve the top quark are unaffected, so our $\Delta S=1$ operators below $m_{t}$ come from

$$
\mathcal{L} \supset-\frac{4 G_{F}}{\sqrt{2}} V^{i d} V^{j s *}\left(\bar{u}_{L}^{i} \gamma^{\mu} d_{L}\right)\left(\bar{s}_{L} \gamma_{\mu} u_{L}^{j}\right)
$$

where now $1 \leq i, j \leq 2$.

For the effective theory below $m_{t}$ to reproduce the theory above $m_{t}$, the effects of the top quark must go into the local $\Delta S=2$ operator. The process of reproducing the results of a theory, using an effective theory with particles integrated out, is known as matching, since we are choosing the coefficient of the $\Delta S=2$ operator so that the results from the five-quark and six-quark theories agree at $m_{t}$. We thus calculate the contributions to $K-\bar{K}$ mixing from the five cases in figure 2.3 where one of the internal lines is a top quark propagator; the finite part of the result will give the coefficient of the $\Delta S=2$ operator in figure 2.4. Using the six-quark effective theory to calculate these diagrams, we obtain the operator

$$
\begin{aligned}
\mathcal{L} & \supset \frac{G_{F}^{2}}{4 \pi^{2}}\left(\xi_{t}^{2} m_{t}^{2}+2 \xi_{t} \xi_{c}\left(m_{t}^{2}+m_{c}^{2}\right)+2 \xi_{t} \xi_{u}\left(m_{t}^{2}+m_{u}^{2}\right)\right)\left(\bar{s}_{L} \gamma^{\mu} d_{L}\right)\left(\bar{s}_{L} \gamma_{\mu} d_{L}\right) \\
& \approx \frac{G_{F}^{2}}{4 \pi^{2}}\left(-\xi_{t}^{2} m_{t}^{2}+2 \xi_{t} \xi_{c} m_{c}^{2}\right)\left(\bar{s}_{L} \gamma^{\mu} d_{L}\right)\left(\bar{s}_{L} \gamma_{\mu} d_{L}\right)
\end{aligned}
$$


where we have used the unitarity of the CKM matrix and the approximation $m_{u} \approx 0$ in the second line, and defined

$$
\xi_{i}=V^{i d} V^{i s *}
$$

\subsubsection{Renormalization Of The $\Delta S=2$ Operator}

Using these two effective operators, we may now calculate the amplitude for $K-$ $\bar{K}$ mixing below the top mass by using only five quarks. However, since the GIM cancellation does not take place between the diagrams responsible for the $\Delta S=2$ operator (because they only make up a subset of the possible diagrams for the mixing), there is a non-trivial counterterm for this operator. The counterterm will depend on the renormalization scale chosen, and leads to the running of the coefficient $c_{2}$ of the operator in equation 2.49. Ignoring QCD renormalization for now, it is clear that the running of $c_{2}$ will be proportional to $c_{1}^{2}$, where $c_{1}=-\frac{4 G_{F}}{\sqrt{2}}$ is the coefficient of the $\Delta S=1$ operator. We omit the calculation of the anomalous dimension, and state the resulting renormalization group equation

$$
M \frac{d}{d M} c_{2}=\frac{1}{8 \pi^{2}} c_{1}^{2} m_{c}^{2} \xi_{c} \xi_{t}
$$

Without QCD renormalization, $c_{1}$ does not run with energy, and from the initial condition

$$
c_{2}\left(m_{t}\right)=\frac{G_{F}^{2}}{4 \pi^{2}}\left(-\xi_{t}^{2} m_{t}^{2}+2 \xi_{t} \xi_{c} m_{c}^{2}\right)
$$

we obtain the solution

$$
c_{2}(M)=\frac{G_{F}^{2}}{4 \pi^{2}}\left(-\xi_{t}^{2} m_{t}^{2}+2 \xi_{t} \xi_{c} m_{c}^{2}\left(1+\log \left(\frac{M^{2}}{m_{t}^{2}}\right)\right)\right)
$$

for $m_{c} \leq M \leq m_{t}$.

So as we run down in energy, the coefficient of the local $\Delta S=2$ operator decreases. 
This is to be expected, since at $M=m_{t}$ the internal top quarks can be produced onshell; conversely, as we move farther away from the pole of the $t$ propagator, virtual top production becomes increasingly difficult.

\subsubsection{Integrating Out $c$}

Finally, we must integrate out the $c$ quark at the energy scale $m_{c}$. Just as before, the set of $\Delta S=1$ operators no longer includes any reference to the quarks that have been integrated out. There is now only one $\Delta S=1$ operator, given by

$$
\mathcal{L} \supset-\frac{4 G_{F}}{\sqrt{2}} V^{u d} V^{u s *}\left(\bar{u}_{L}^{1} \gamma^{\mu} d_{L}\right)\left(\bar{s}_{L} \gamma_{\mu} u_{L}^{1}\right)
$$

Consequently, the $K-\bar{K}$ mixing contributions involving the $c$ quark that arose from two insertions of the $\Delta S=1$ operator must be absorbed into the $\Delta S=2$ operator. Adding these contributions to the five-quark theory result for $c_{2}\left(m_{c}\right)$, we have

$$
\begin{aligned}
c_{2} & =\frac{G_{F}^{2}}{4 \pi^{2}}\left(-\xi_{t}^{2} m_{t}^{2}+2 \xi_{t} \xi_{c} m_{c}^{2}\left(1+\log \left(\frac{m_{c}^{2}}{m_{t}^{2}}\right)\right)+\xi_{c}^{2} m_{c}^{2}+2 \xi_{c} \xi_{u}\left(m_{c}^{2}+m_{u}^{2}\right)\right) \\
& \approx \frac{G_{F}^{2}}{4 \pi^{2}}\left(-\xi_{t}^{2} m_{t}^{2}+2 \xi_{t} \xi_{c} m_{c}^{2}\left(1+\log \left(\frac{m_{c}^{2}}{m_{t}^{2}}\right)\right)+\xi_{c}^{2} m_{c}^{2}+2 \xi_{c} \xi_{u} m_{c}^{2}\right) \\
& =-\frac{G_{F}^{2}}{4 \pi^{2}}\left(\xi_{t}^{2} m_{t}^{2}+\xi_{c}^{2} m_{c}^{2}+2 \xi_{t} \xi_{c} m_{c}^{2} \log \left(\frac{m_{t}^{2}}{m_{c}^{2}}\right)\right),
\end{aligned}
$$

where we have again taken $m_{u} \approx 0$ and used the unitarity of the CKM matrix.

We may now calculate the full amplitude for $K-\bar{K}$ mixing at low energies. The only remaining contribution below $m_{c}$ resulting from two insertions of the $\Delta S=1$ operator has two $u$ propagators, and is proportional to $m_{u}$. If the up quark is taken to be approximately massless, then this contribution vanishes. The amplitude is thus 
trivially given by the $\Delta S=2$ operator, and is

$$
\mathcal{M}=-i \frac{G_{F}^{2}}{4 \pi^{2}}\left(\xi_{t}^{2} m_{t}^{2}+\xi_{c}^{2} m_{c}^{2}+2 \xi_{t} \xi_{c} m_{c}^{2} \log \left(\frac{m_{t}^{2}}{m_{c}^{2}}\right)\right)\left(\bar{s}_{L} \gamma^{\mu} d_{L}\right)\left(\bar{s}_{L} \gamma_{\mu} d_{L}\right)
$$

which is in agreement with the calculation in the full theory [5].

\subsubsection{QCD Corrections}

We now consider how QCD corrections are implemented in the effective field theory. First, the coefficient of the $\Delta S=1$ operator now runs with energy, and we take

$$
M \frac{d}{d M} c_{1}=\gamma_{1} c_{1}
$$

for some anomalous dimension $\gamma_{1}$. This is a simplification; since there are different $\Delta S=1$ operators with the same quantum numbers, the above equation should actually be replaced with a matrix equation involving mixing of the different operators as the energy scale is varied. This computation is significantly more involved, but has been done [12].

As well, the renormalization group equation for $c_{2}$ now has an additional term from similar QCD corrections:

$$
M \frac{d}{d M} c_{2}=\frac{1}{8 \pi^{2}} c_{1}^{2} m_{c}^{2} \xi_{c} \xi_{t}+\gamma_{2} c_{2}
$$

Finally, after we choose to perform the first matching at the scale $m_{t}$, the energy scale of the second matching is subject to renormalization. There is thus one last renormalization group equation,

$$
M \frac{d}{d M} m_{c}=\gamma_{m} m_{c}
$$


for the matching scale $m_{c}$. The running of the quark mass operator, which yields $\gamma_{m}$, is computed explicitly in Peskin [6].

Note that the anomalous dimensions may all be computed relatively easily. They each involve, at lowest order, one loop gluon exchange diagrams. However, this is not the case in the full theory, demonstrating one of the major advantages of effective field theories. The box diagram of figure 2.2 already has one loop, and so a QCD correction generally involves a two loop calculation.

In summary, at the price of neglecting terms of higher order in $\frac{1}{m_{W}}$, we have found the amplitude for $K-\bar{K}$ mixing through a few relatively simple calculations, not needing to compute the full box diagram. In addition, the QCD corrections to the amplitude may be readily obtained in the effective theory, whereas in the full theory, they are much more difficult to extract. We will use these techniques in the next chapter to find the QCD enhancement factor of an effective Lagrangian term, regardless of the details of the underlying physics. 


\section{References}

[1] K. G. Wilson and John B. Kogut. The renormalization group and the epsilon expansion. Phys. Rept., 12:75-200, 1974.

[2] K. G. Wilson. The renormalization group and critical phenomena. Rev. Mod. Phys., 55:583-600, 1983.

[3] Joseph Polchinski. Effective field theory and the Fermi surface. 1992. hepth/9210046.

[4] David B. Kaplan. Effective field theories. 1995. nucl-th/9506035.

[5] Aneesh V. Manohar. Effective field theories. 1996. hep-ph/9606222.

[6] Michael Edward Peskin and Daniel V. Schroeder. An introduction to quantum field theory. Reading, USA: Addison-Wesley (1995) $842 \mathrm{p}$.

[7] W.-M. Yao et al. Review of particle physics. J. Phys., G33:1-1232, 2006.

[8] Jr. Callan, Curtis G. Broken scale invariance in scalar field theory. Phys. Rev., D2:1541-1547, 1970.

[9] K. Symanzik. Small distance behavior in field theory and power counting. Commun. Math. Phys., 18:227-246, 1970.

[10] E. Fermi. An attempt of a theory of beta radiation. Z. Phys., 88:161-177, 1934.

[11] Frederick J. Gilman and Mark B. Wise. Strong interaction corrections to K0 anti-K0 mixing in the six quark model. Phys. Lett., B93:129, 1980.

[12] Frederick J. Gilman and Mark B. Wise. K0 anti-K0 mixing in the six quark model. Phys. Rev., D27:1128, 1983.

[13] S. L. Glashow, J. Iliopoulos, and L. Maiani. Weak interactions with leptonhadron symmetry. Phys. Rev., D2:1285-1292, 1970. 


\section{Chapter 3}

\section{Leptonic Pion Decay And Physics Beyond The Standard Model}

\subsection{Introduction}

In the Standard Model, the decay of the charged pion is chirally suppressed due to the $V-A$ structure of the weak interaction. (For a review of chiral suppression in pion decay, see Griffiths [1].) Measurement of the ratio of branching ratios $\frac{\Gamma(\pi \rightarrow e \nu)}{\Gamma(\pi \rightarrow \mu \nu)}$ thus serves as a test of new physics. In particular, various models predict additional pseudoscalar interactions, to which this ratio is particularly sensitive. These include R-parity violating supersymmetry, leptoquarks, certain (super)compositeness models, and extra Higgs theories. Given the good agreement between the Standard Model prediction for $\frac{\Gamma(\pi \rightarrow e \nu)}{\Gamma(\pi \rightarrow \mu \nu)}$ and experiment, limits can be placed on the strengths of these interactions $[2,3,4,5,6,7,8,9,10,11,12,13,14,15,16,17,18,19,20,21,22,23,24$, $25,26,27,28,29]$. These limits compete against those obtained from processes such as beta decay [30]. However, experiments at TRIUMF [31] and PSI [32] should reduce the uncertainty on the experimental $R_{\pi}$, which is currently 40 times greater than its theoretical counterpart, by about one order of magnitude. With this improvement, pion decay should yield better limits than beta decay for the interactions we consider 
in this chapter.

We calculate, with new QCD corrections, the effect of an additional pseudoscalar interaction on the pion branching ratio. We then use the result to constrain different models of new physics, and compare our findings with the existing literature. Finally, we discuss our conclusions in light of the prospect of new pion decay experiments.

\subsection{Effect of Pseudoscalar Interaction}

Consider the ratio

$$
R_{\pi}=\frac{\Gamma(\pi \rightarrow e \nu)}{\Gamma(\pi \rightarrow \mu \nu)}
$$

Additional pseudoscalar interactions

$$
\mathcal{L}_{\text {eff }} \supset-i \frac{\rho_{e}}{2 \Lambda^{2}}\left[\bar{e}\left(1-\gamma_{5}\right) \nu_{e}\right]\left[\bar{u} \gamma_{5} d\right]-i \frac{\rho_{\mu}}{2 \Lambda^{2}}\left[\bar{\mu}\left(1-\gamma_{5}\right) \nu_{\mu}\right]\left[\bar{u} \gamma_{5} d\right]
$$

where $\rho_{e}$ and $\rho_{\mu}$ are real, would change this ratio to approximately

$$
R_{\pi} \approx R_{\pi}(\mathrm{SM})\left(1+\sqrt{2} \frac{\tilde{f}_{\pi} \rho_{e}}{G_{F} \Lambda^{2} f_{\pi} m_{e}}-\sqrt{2} \frac{\tilde{f}_{\pi} \rho_{\mu}}{G_{F} \Lambda^{2} f_{\pi} m_{\mu}}\right)
$$

where $f_{\pi}=93 \mathrm{MeV}$ is the pion decay constant and $\tilde{f}_{\pi}=-\frac{i}{\sqrt{2}}\left\langle 0\left|\bar{u} \gamma^{5} d\right| \pi^{-}\right\rangle=$ $\frac{m_{\pi}^{2}}{m_{u}+m_{d}} f_{\pi}=1.8 \cdot 10^{5} \mathrm{MeV}^{2}[33]$. We have only kept the terms of lowest order in the couplings $\rho_{e}$ and $\rho_{\mu}$. The best current theoretical estimate for this ratio in the Standard Model is $R_{\pi}(\mathrm{SM})=(1.2352 \pm 0.0001) \cdot 10^{-4}$, and was obtained by calculating QED radiative corrections to the decay [34] and considering higher order terms in the QCD chiral Lagrangian [35]. Conversely, experiments measure $R_{\pi}=(1.230 \pm 0.004) \cdot 10^{-4}$ [36]. Combining the errors and taking the weakest limit, we find that at $2 \sigma$,

$$
\left|\sqrt{2} \frac{\tilde{f}_{\pi} \rho_{e}}{G_{F} \Lambda^{2} f_{\pi} m_{e}}-\sqrt{2} \frac{\tilde{f}_{\pi} \rho_{\mu}}{G_{F} \Lambda^{2} f_{\pi} m_{\mu}}\right| \leq 1.07 \cdot 10^{-2}
$$


We now calculate the one-loop QCD correction to this limit, still treating the additional interaction as a four-fermion local operator. The anomalous dimension of the operator $\left[\bar{e}\left(1-\gamma_{5}\right) \nu_{e}\right]\left[\bar{u} \gamma_{5} d\right]$ is $\gamma=-\frac{2}{\pi} \alpha_{s}$ (see the calculation in appendix B). We may use this anomalous dimension to compute the QCD enhancement of a pseudoscalar contribution to pion decay, regardless of the underlying physics. Note that using equation 2.31, we may rewrite equation 2.39 for the running of an operator coefficient as

$$
c\left(p_{1}\right)=\left(\frac{\alpha\left(p_{1}\right)}{\alpha\left(p_{2}\right)}\right)^{\frac{a_{O}}{2 b_{0}}} c\left(p_{2}\right)
$$

For the pseudoscalar pion decay operator, $a_{\mathcal{O}}=8$. Now, assume that the new physics responsible for this operator occurs at a scale $\Lambda \sim 1 \mathrm{TeV}$. From $\Lambda$ down to $m_{t}$, we have six quarks, so

$$
b_{0}=11-\frac{2}{3}(6)=7,
$$

using the QCD beta function of equation 2.40. QCD running from $\Lambda$ to $m_{t}$ thus enhances the operator by a factor of

$$
\frac{c\left(m_{t}\right)}{c(\Lambda)}=\left(\frac{\alpha_{s}\left(m_{t}\right)}{\alpha_{s}(\Lambda)}\right)^{\frac{4}{7}}
$$

Note that since $\alpha_{s}$ decreases with increasing energy, this is indeed an enhancement. From $m_{t}$ down to $m_{b}$, the renormalization group running again enhances the pseudoscalar operator. However, since the top quark is integrated out below $m_{t}$, we must now use

$$
b_{0}=11-\frac{2}{3}(5)=\frac{23}{3} .
$$

This procedure may be continued all the way down to the scale $4 \pi f_{\pi}$, where QCD becomes strongly coupled [37] [38]. At this point, perturbation theory no longer provides a good description of the physics, and thus cannot predict the renormalization group running of the pseudoscalar pion decay operator. 
By multiplying the enhancement factors from each stage of the running, however, we can at least obtain a partial estimate of the effects of renormalization on our limits. We find that the leading QCD corrections will enhance each bound by a factor of

$$
\left(\frac{\alpha_{s}\left(4 \pi f_{\pi}\right)}{\alpha_{s}\left(m_{c}\right)}\right)^{4 / 9}\left(\frac{\alpha_{s}\left(m_{c}\right)}{\alpha_{s}\left(m_{b}\right)}\right)^{12 / 25}\left(\frac{\alpha_{s}\left(m_{b}\right)}{\alpha_{s}\left(m_{t}\right)}\right)^{12 / 23}\left(\frac{\alpha_{s}\left(m_{t}\right)}{\alpha_{s}(\Lambda)}\right)^{4 / 7}
$$

where $\Lambda$ is the scale at which the interaction occurs. Each factor may be computed numerically using the renormalization group equation for $\alpha_{s}$, given the value of $\alpha_{s}$ at some reference point. We take $\alpha_{s}\left(m_{Z}\right)=0.1176, m_{c}=1.27 \mathrm{GeV}, m_{b}=4.20 \mathrm{GeV}$, and $m_{t}=171.2 \mathrm{GeV}$ [36], and run $\alpha_{s}$ using equations 2.29 and 2.40. We also choose $\Lambda=1 \mathrm{TeV}$, to obtain a conservative lower bound on the QCD enhancement of any new operator. (Although the enhancement would be greater if $\Lambda$ were increased, the difference would be minimal, since $\alpha_{s}$ runs very slowly at high energies.) Since the result is approximately 2.0 , the net effect of the renormalization group flow has been to double the significance of any pseudoscalar contribution to pion decay. Conversely, given an experimental limit on the size of the pseudoscalar operator contribution to pion decay, our computation of the QCD running will allow us to place limits on possible sources of pseudoscalar contributions to pion decay that are twice as strong. The new limit is thus given by

$$
\left|\sqrt{2} \frac{\tilde{f}_{\pi} \rho_{e}}{G_{F} \Lambda^{2} f_{\pi} m_{e}}-\sqrt{2} \frac{\tilde{f}_{\pi} \rho_{\mu}}{G_{F} \Lambda^{2} f_{\pi} m_{\mu}}\right| \leq 5.3 \cdot 10^{-3}
$$

The additional interactions that we have considered so far interfere coherently with the Standard Model amplitude for pion decay, and the strength of the limit in equation 3.10 is partly due to this interference. However, we may also bound 
interactions that add incoherently with pion decay. For instance, the terms

$$
-i \frac{\rho_{e}^{\prime}}{2 \Lambda^{2}}\left[\bar{e}\left(1-\gamma_{5}\right) \nu_{x}\right]\left[\bar{u} \gamma_{5} d\right]-i \frac{\rho_{\mu}^{\prime}}{2 \Lambda^{2}}\left[\bar{\mu}\left(1-\gamma_{5}\right) \nu_{y}\right]\left[\bar{u} \gamma_{5} d\right]
$$

where $x \neq e$ and $y \neq \mu$, contribute to pion decay but only add incoherently with the Standard Model amplitude. However, the lack of chiral suppression still allows us to put competitive bounds on some of these terms. These interactions would change the pion branching ratio to [33]

$$
R_{\pi} \approx R_{\pi}(\mathrm{SM})\left(1+\frac{\tilde{f}_{\pi}^{2} \rho_{e}^{\prime 2}}{2 G_{F}^{2} \Lambda^{4} f_{\pi}^{2} m_{e}^{2}}-\frac{\tilde{f}_{\pi}^{2} \rho_{\mu}^{\prime 2}}{2 G_{F}^{2} \Lambda^{4} f_{\pi}^{2} m_{\mu}^{2}}\right)
$$

Repeating the above analysis, we obtain the limit

$$
\left|\frac{\tilde{f}_{\pi}^{2} \rho_{e}^{\prime 2}}{2 G_{F}^{2} \Lambda^{4} f_{\pi}^{2} m_{e}^{2}}-\frac{\tilde{f}_{\pi}^{2} \rho_{\mu}^{\prime 2}}{2 G_{F}^{2} \Lambda^{4} f_{\pi}^{2} m_{\mu}^{2}}\right| \leq 5.3 \cdot 10^{-3}
$$

\subsection{Limits}

\subsubsection{R-Parity Violating Supersymmetry}

As described in section 2.1.3, corrections to the Higgs propagator from the particles in the Standard Model cause divergent shifts in the Higgs mass, which should be cut off at the scale of new physics. If there is no new physics until the Planck scale, then an extremely precise cancellation would have to take place between the Higgs mass corrections to give the mass that is implied by experimental data. Now, fermions and bosons lead to Higgs mass corrections of opposite sign. Supersymmetry exploits this sign difference to resolve the hierarchy problem by proposing a symmetry between fermions and bosons. In supersymmetric models, for each Standard Model fermion, there is a boson, and vice versa. The so-called superpartners have the same quantum 
numbers as the original particles, except for spin.

The superfield formalism is used to describe supersymmetric theories in terms of superfields, each containing a Standard Model field with its superpartner (along with an auxiliary field) $[39,40]$. Using this formalism, we may construct the superpotential

$$
W=u_{i j}\left(Q_{L}^{i} H_{u}\right) U_{L}^{j, c}+d_{i j}\left(Q_{L}^{i} H_{d}\right) D_{L}^{j, c}+e_{i j}\left(L_{L}^{i} H_{d}\right) E_{L}^{j, c}+\mu H_{u} H_{d}
$$

where the superfield $Q_{L}^{i}$ contains the left-handed $i$ th-generation quark $S U(2)$ doublet, $E_{L}^{j, c}$ contains the conjugate of the right-handed $j$ th-generation charged lepton $S U(2)$ singlet, etc. Superpotentials are always holomorphic functions of the superfields; i.e., they never contain the complex conjugates of superfields. We have used parentheses to indicate implicit contraction of $S U(2)$ indices, and omitted $S U(3)$ indices. Note that supersymmetric models have two doublets of Higgs fields; here, they are denoted by $H_{u}$ and $H_{d}$, for the quarks to which they couple. $W$ is the superpotential for the Minimal Supersymmetric Standard Model (MSSM), the simplest extension of the Standard Model incorporating supersymmetry. From $W$, we may extract the Standard Model Lagrangian, along with additional interaction terms involving the superpartners [41]. If the superpotential involves the scalar fields $\phi_{i}$, each having fermionic superpartners $\psi_{i}$, then the Lagrangian terms arising from the superpotential, known as F-terms, are given by

$$
\mathcal{L} \supset-\frac{1}{2} \sum_{i, j} \frac{\partial^{2} W}{\partial \phi_{i} \partial \phi_{j}}\left(\psi_{i} \psi_{j}\right)+\text { h.c. }
$$

However, the MSSM superpotential does not contain all of the possible terms allowed by gauge invariance $[39,41]$. In particular, the $L_{L}$ and $H_{d}$ superfields have the same gauge quantum numbers, and we may thus make the substitution $H_{d} \rightarrow L_{L}$ in any allowed superpotential term to obtain another allowed superpotential term. 
This substitution may be performed on the second and third terms in the MSSM superpotential above. With the last term, the substitution gives an unphysical, after field redefinition, bilinear coupling between leptons and Higgs fields, which we omit. Also, a $U_{L}^{c} D_{L}^{c} D_{L}^{c}$ term is not forbidden by any of the gauge symmetries. The additional superpotential terms that we consider are thus of the form

$$
\lambda_{i j k}\left(L_{L}^{i} L_{L}^{j}\right) E_{L}^{k, c}+\lambda_{i j k}^{\prime}\left(L_{L}^{i} Q_{L}^{j}\right) D_{L}^{k, c}+\lambda_{i j k}^{\prime \prime} U_{L}^{i, c} D_{L}^{j, c} D_{L}^{k, c}
$$

where again $S U(2)$ singlets are indicated by parentheses and $S U(3)$ indices are omitted. Note that by the $S U(2)$ and $S U(3)$ gauge symmetries, respectively, we have $\lambda_{i j k}=-\lambda_{j i k}$ and $\lambda_{i j k}^{\prime \prime}=-\lambda_{i k j}^{\prime \prime}$. Each of the R-parity violating terms violates either baryon number or lepton number. (Note that these are global symmetries, not gauge symmetries.) To preserve these symmetries, which prevent experimentally constrained processes such as proton decay from occurring, the R-parity violating terms are usually eliminated by imposing a discrete $Z_{2}$ symmetry called $R$-parity, under which a particle has charge

$$
R=(-1)^{3 B+L+2 S}
$$

where $B, L$, and $S$ are baryon number, lepton number, and spin, respectively. With this definition, all Standard Model particles have $R=1$, and their superpartners have $R=-1$.

If the $B$-violating $U_{L}^{c} D_{L}^{c} D_{L}^{c}$ and $L$-violating $\left(L_{L} Q_{L}\right) D_{L}^{c}$ terms are both allowed, proton decay places extremely stringent constraints on the products $\lambda^{\prime} \lambda^{\prime \prime}$ [42]. Other bounds involving the $B$-violating couplings are discussed in Carlson et al. [43]; in particular, when the $U_{L}^{c} D_{L}^{c} D_{L}^{c}$ and $\left(L_{L} L_{L}\right) E_{L}^{c}$ terms are allowed, proton decay still yields excellent constraints on most products of couplings. We consider only the $L$-violating terms, setting $\lambda_{i j k}^{\prime \prime}=0$. Under different coupling structures, two types of contributions to pion decay are possible, shown in figures 3.1 and 3.2. We assume the double 


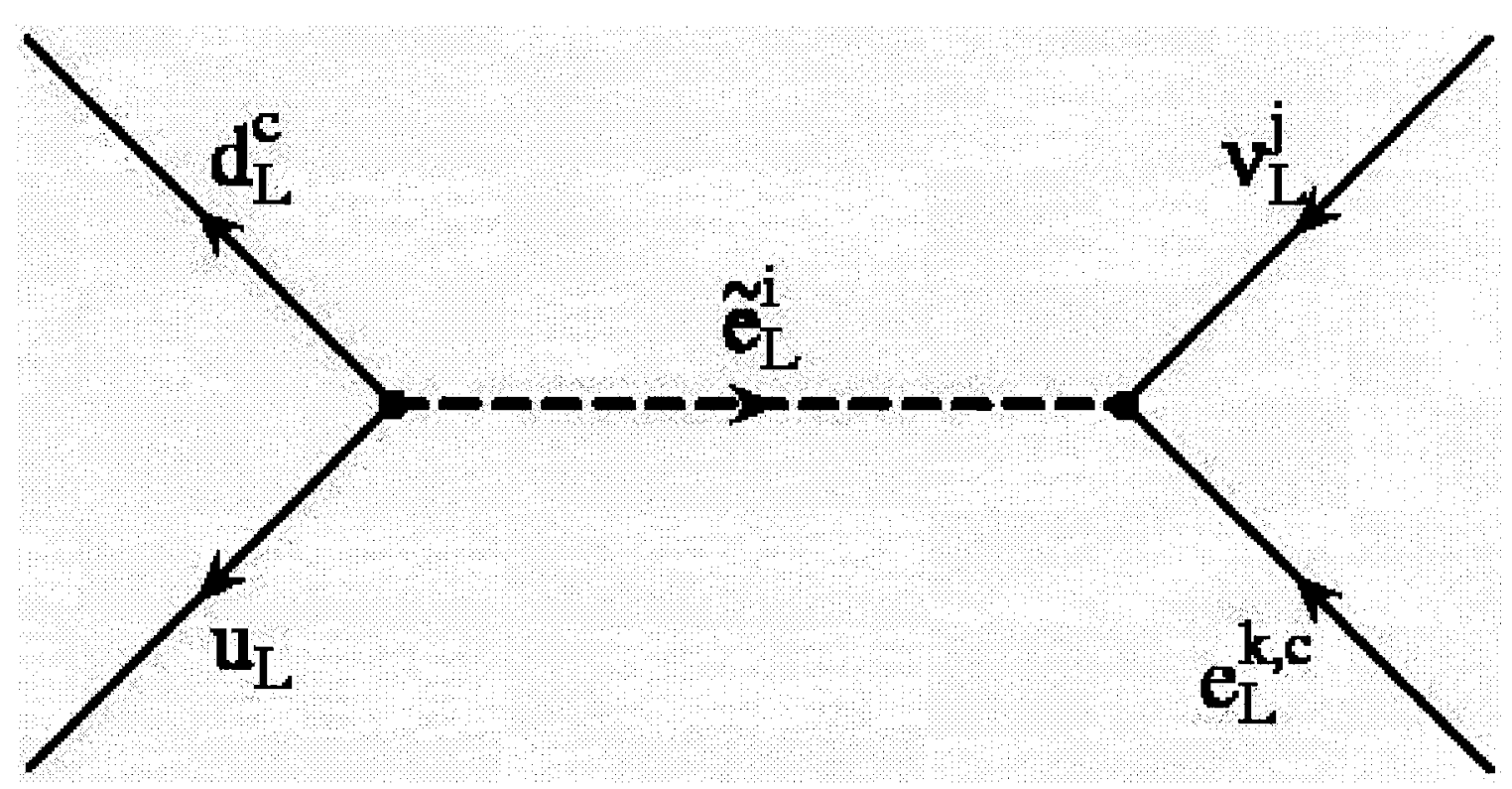

Figure 3.1: An R-parity violating SUSY contribution to pion decay. The superpartner of the left-handed $i$ th generation charged lepton is denoted by $\tilde{e}_{L}^{i}$.

coupling dominance hypothesis, considering only two couplings to be significant at a time. This assumption allows to apply our general limits from the previous section to each new contribution separately, ignoring cancellations between the contributions.

Two types of diagrams are allowed by the new superpotential terms. The first ${ }^{1}$, shown in figure 3.1 , has effective strength

$$
\begin{aligned}
\rho_{k} & =\frac{\operatorname{Re}\left(\lambda_{i k k} \lambda_{i 11}^{\prime *}\right)}{2} \\
\rho_{k}^{\prime} & =\frac{\operatorname{Re}\left(\lambda_{i j k} \lambda_{i 11}^{\prime *}\right)}{2}
\end{aligned}
$$

where $\lambda$ and $\lambda^{\prime}$ are the couplings at the vertices, and in the second equation $j \neq k$. From this, we can obtain a limit on the product of couplings in terms of the mass of the mediating particle. The limits obtained in this manner are shown in table 3.1.

\footnotetext{
${ }^{1}$ The existence of which was mentioned in [16], but the resulting limits are calculated here for the first time.
} 


\begin{tabular}{|c|c|}
\hline Product of couplings & Limit \\
\hline $\operatorname{Re}\left(\lambda_{121} \lambda_{111}^{\prime *}\right)$ & $6.3 \times 10^{-6}\left[\tilde{e}_{1}\right]^{2}$ \\
$\operatorname{Re}\left(\lambda_{122} \lambda_{111}^{\prime *}\right)$ & $4.8 \times 10^{-5}\left[\tilde{e}_{1}\right]^{2}$ \\
$\operatorname{Re}\left(\lambda_{121} \lambda_{211}^{\prime *}\right)$ & $2.3 \times 10^{-7}\left[\tilde{e}_{2}\right]^{2}$ \\
$\operatorname{Re}\left(\lambda_{122} \lambda_{211}^{\prime *}\right)$ & $1.3 \times 10^{-3}\left[\tilde{e}_{2}\right]^{2}$ \\
$\operatorname{Re}\left(\lambda_{131} \lambda_{111}^{\prime *}\right)$ & $6.3 \times 10^{-6}\left[\tilde{e}_{1}\right]^{2}$ \\
$\operatorname{Re}\left(\lambda_{132} \lambda_{111}^{\prime *}\right)$ & $1.3 \times 10^{-3}\left[\tilde{e}_{1}\right]^{2}$ \\
$\operatorname{Re}\left(\lambda_{131} \lambda_{311}^{\prime *}\right)$ & $2.3 \times 10^{-7}\left[\tilde{e}_{3}\right]^{2}$ \\
$\operatorname{Re}\left(\lambda_{132} \lambda_{311}^{\prime *}\right)$ & $1.3 \times 10^{-3}\left[\tilde{e}_{3}\right]^{2}$ \\
$\operatorname{Re}\left(\lambda_{231} \lambda_{211}^{\prime *}\right)$ & $6.3 \times 10^{-6}\left[\tilde{e}_{2}\right]^{2}$ \\
$\operatorname{Re}\left(\lambda_{232} \lambda_{211}^{\prime *}\right)$ & $1.3 \times 10^{-3}\left[\tilde{e}_{2}\right]^{2}$ \\
$\operatorname{Re}\left(\lambda_{231} \lambda_{311}^{\prime *}\right)$ & $6.3 \times 10^{-6}\left[\tilde{e}_{3}\right]^{2}$ \\
$\operatorname{Re}\left(\lambda_{232} \lambda_{311}^{\prime *}\right)$ & $4.8 \times 10^{-5}\left[\tilde{e}_{3}\right]^{2}$ \\
\hline
\end{tabular}

Table 3.1: Limits on R-parity violating SUSY coupling combinations $\operatorname{Re}\left(\lambda \lambda^{\prime *}\right)$ from slepton exchange. We use $[\tilde{e}]$ to represent $\frac{m_{\tilde{e}}}{100 \mathrm{GeV}}$.

Note that throughout our results, we use $[\tilde{q}]$ to represent $\frac{m_{\tilde{q}}}{100 \mathrm{GeV}}$.

We may obtain another effective contribution to pion decay by Fierz reordering (see appendix C) an interaction arising purely from the $L_{L} Q_{L} D_{L}^{c}$ couplings, shown in figure 3.2. Although this contribution has already been considered [13, 26, 27], we update the limits using current experimental data and the QCD correction. The Fierz reordering weakens the bound, since there is now chiral suppression. We have

$$
R_{\pi} \approx R_{\pi}(\mathrm{SM})\left(1+\left(\frac{1}{2 \sqrt{2} G_{F} m^{2}}\right)\left(\left|\lambda_{11 i}^{\prime}\right|^{2}-\left|\lambda_{21 i}^{\prime}\right|^{2}\right)\right)
$$

where $m$ is the mass of the mediating down squark. The resulting limits are shown in table 3.2. These bounds are somewhat better than those previously obtained in the literature [27]. 


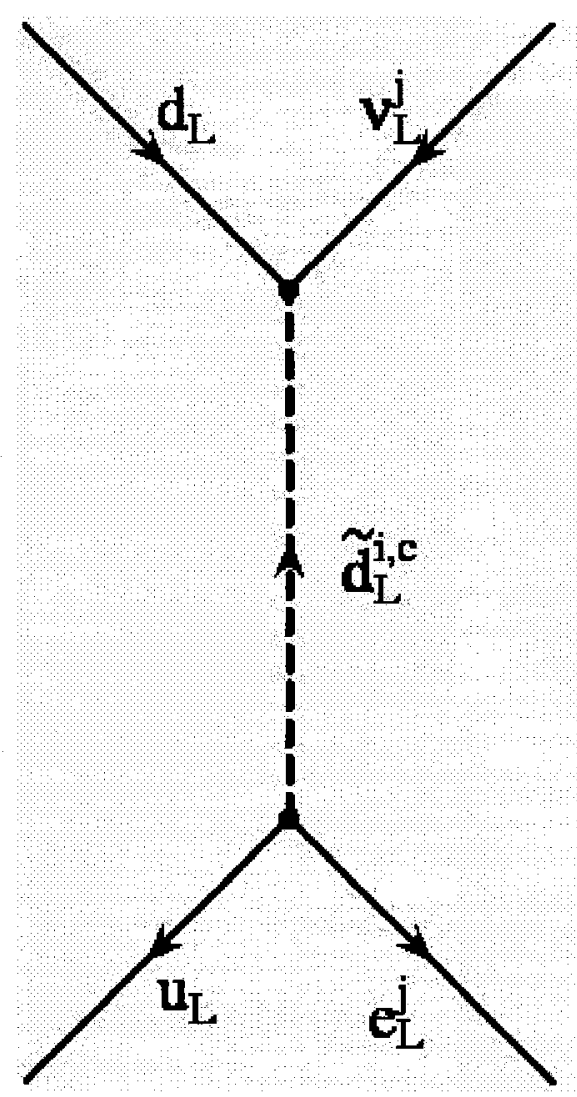

Figure 3.2: Another R-parity violating SUSY contribution to pion decay, which has been considered before [13]. The superpartner of the left-handed $i$ th generation down quark is denoted by $\tilde{d}_{L}^{i}$.

\begin{tabular}{|c|c|c|}
\hline Product of couplings & New limit & Previous limit from [27] \\
\hline$\left|\lambda_{111}^{\prime}\right|^{2}$ & $1.7 \times 10^{-3}\left[\tilde{d}_{1}\right]^{2}$ & $3.3 \times 10^{-3}\left[\tilde{d}_{1}\right]^{2}$ \\
$\left|\lambda_{112}^{\prime}\right|^{2}$ & $1.7 \times 10^{-3}\left[\tilde{d}_{2}\right]^{2}$ & $3.3 \times 10^{-3}\left[\tilde{d}_{2}\right]^{2}$ \\
$\left|\lambda_{113}^{\prime}\right|^{2}$ & $1.7 \times 10^{-3}\left[\tilde{d}_{3}\right]^{2}$ & $3.3 \times 10^{-3}\left[\tilde{d}_{3}\right]^{2}$ \\
$\left|\lambda_{211}^{\prime}\right|^{2}$ & $1.7 \times 10^{-3}\left[\tilde{d}_{1}\right]^{2}$ & $5.9 \times 10^{-3}\left[\tilde{d}_{1}\right]^{2}$ \\
$\left|\lambda_{212}^{\prime}\right|^{2}$ & $1.7 \times 10^{-3}\left[\tilde{d}_{2}\right]^{2}$ & $5.9 \times 10^{-3}\left[\tilde{d}_{2}\right]^{2}$ \\
$\left|\lambda_{213}^{\prime}\right|^{2}$ & $1.7 \times 10^{-3}\left[\tilde{d}_{3}\right]^{2}$ & $5.9 \times 10^{-3}\left[\tilde{d}_{3}\right]^{2}$ \\
\hline
\end{tabular}

Table 3.2: Updated limits on R-parity violating SUSY couplings $\left|\lambda^{\prime}\right|^{2}$ from squark exchange. We use $[\tilde{q}]$ to represent $\frac{m_{\tilde{q}}}{100 \mathrm{GeV}}$. 


\subsubsection{Leptoquarks}

Leptoquarks, particles that couple to both leptons and quarks, appear in various extensions of the Standard Model, including Grand Unified Theories [44, 45, 46] and technicolour models [47]. If we require that the Lagrangian remains invariant under $S U(3) \times S U(2) \times U(1)$ transformations, but allow violation of the global symmetries corresponding to baryon and lepton number, we may write the new terms [11]

$$
\begin{aligned}
\mathcal{L} & \supset\left(\lambda_{L S_{0}}^{i j} \bar{Q}_{L}^{j, c} i \sigma^{2} L_{L}^{i}+\lambda_{R S_{0}}^{i j} \bar{u}_{R}^{j, c} e_{R}^{i}\right) S_{0}^{\dagger}+\lambda_{R \tilde{S}_{0}}^{i j} \bar{d}_{R}^{j, c} e_{R}^{i} \tilde{S}_{0}^{\dagger} \\
& +\left(\lambda_{L S_{1 / 2}}^{i j} \bar{u}_{R}^{j} L_{L}^{i}+\lambda_{R S_{1 / 2}}^{i j} \bar{Q}_{L}^{j} i \sigma^{2} e_{R}^{i}\right) S_{1 / 2}^{\dagger}+\lambda_{L \tilde{S}_{1 / 2}}^{i j} \bar{d}_{R}^{j} L_{L}^{i} \tilde{S}_{1 / 2}^{\dagger}+\lambda_{L S_{1}}^{i j} \bar{Q}_{L}^{j, c} i \sigma^{2} \sigma^{a} L_{L}^{i} S_{1}^{a \dagger} \\
& +\left(\lambda_{L V_{0}}^{i j} \bar{Q}_{L}^{j} \gamma_{\mu} L_{L}^{i}+\lambda_{R V_{0}}^{i j} \bar{d}_{R}^{j} \gamma_{\mu} e_{R}^{i}\right) V_{0}^{\mu \dagger}+\lambda_{R \tilde{V}_{0}}^{i j} \bar{u}_{R}^{j} \gamma_{\mu} e_{R}^{i} \tilde{V}_{0}^{\mu \dagger} \\
& +\left(\lambda_{L V_{1 / 2}}^{i j} \bar{d}_{R}^{j, c} \gamma_{\mu} L_{L}^{i}+\lambda_{R V_{1 / 2}}^{i j} \bar{Q}_{L}^{j, c} \gamma_{\mu} e_{R}^{i}\right) V_{1 / 2}^{\mu \dagger}+\lambda_{L \tilde{V}_{1 / 2}}^{i j} \bar{u}_{R}^{j, c} \gamma_{\mu} L_{L}^{i} \tilde{V}_{1 / 2}^{\mu \dagger} \\
& +\lambda_{L V_{1}}^{i j} \bar{Q}_{L}^{j} \gamma_{\mu} \sigma^{a} L_{L}^{i} V_{1}^{a \mu \dagger}+\text { h.c. }
\end{aligned}
$$

where $X^{c}$ is the conjugate of the field $X$, and barred fields are outgoing. (Note that in this convention, which we have adopted from [11], $u_{R}^{c}$ is the conjugate of the $u_{R}$ field, which is left-handed. This differs from the convention used everywhere else in this work, where $u_{R}^{c}$ is the right-handed up anti-quark field.) A summary of the leptoquarks and their quantum numbers is given in table 3.3. We have explicitly indicated the $S U(2)$ algebra of weak isospin using the Pauli matrices.

Leptoquarks can provide both pseudoscalar and axial-vector contributions to pion decay. Both of these types of contributions interfere with the Standard Model amplitude, but the axial-vector contribution is still chirally suppressed, and will thus lead to less stringent bounds. Here, we update the previous limits on leptoquark couplings, using the latest experimental data for $R_{\pi}$ and including the QCD correction factor. 


\begin{tabular}{|c|c|c|c|}
\hline Leptoquark & Spin & Charge & Hypercharge \\
\hline$S_{0}$ & 0 & $-\frac{1}{3}$ & $-\frac{2}{3}$ \\
$\tilde{S}_{0}$ & 0 & $-\frac{4}{3}$ & $-\frac{8}{3}$ \\
$S_{1 / 2}$ & 0 & $-\frac{2}{3},-\frac{5}{3}$ & $-\frac{7}{3}$ \\
$\tilde{S}_{1 / 2}$ & 0 & $\frac{1}{3},-\frac{2}{3}$ & $-\frac{1}{3}$ \\
$S_{1}$ & 0 & $-\frac{2}{3},-\frac{1}{3},-\frac{4}{3}$ & $-\frac{2}{3}$ \\
$V_{0}$ & 1 & $-\frac{2}{3}$ & $-\frac{4}{3}$ \\
$\tilde{V}_{0}$ & 1 & $-\frac{5}{3}$ & $-\frac{10}{3}$ \\
$V_{1 / 2}$ & 1 & $-\frac{1}{3},-\frac{4}{3}$ & $-\frac{5}{3}$ \\
$\tilde{V}_{1 / 2}$ & 1 & $\frac{2}{3},-\frac{1}{3}$ & $\frac{1}{3}$ \\
$V_{1}$ & 1 & $\frac{1}{3},-\frac{2}{3},-\frac{5}{3}$ & $-\frac{4}{3}$ \\
\hline
\end{tabular}

Table 3.3: Quantum numbers of the leptoquarks in equation 3.20.

Pseudoscalar leptoquark contributions to pion decay arise from the effective fourfermion interactions

$$
\begin{aligned}
\mathcal{L}_{\mathrm{eff}} & \supset \frac{\lambda_{L S_{0}}^{i j} \lambda_{R S_{0}}^{k l *}}{2 m_{S_{0}}^{2}}\left(\bar{d}_{L}^{j, c} u_{R}^{l, c}\right)\left(\bar{e}_{R}^{k} \nu_{L}^{i}\right) \\
+ & \frac{\lambda_{L S_{1 / 2}}^{i j} \lambda_{R S_{1 / 2}}^{k l *}}{2 m_{S_{1 / 2}}^{2}}\left(\bar{u}_{R}^{j} d_{L}^{l}\right)\left(\bar{e}_{R}^{k} \nu_{L}^{i}\right) \\
+ & \frac{\lambda_{L V_{0}}^{i j} \lambda_{R V_{0}}^{k l *}}{m_{V_{0}}^{2}}\left(\bar{u}_{L}^{j} d_{R}^{l}\right)\left(\bar{e}_{R}^{k} \nu_{L}^{i}\right) \\
+ & \frac{\lambda_{L V_{1 / 2}}^{i j} \lambda_{R V_{1 / 2}}^{k l *}}{m_{V_{1 / 2}}^{2}}\left(\bar{d}_{R}^{j, c} u_{L}^{l, c}\right)\left(\bar{e}_{R}^{k} \nu_{L}^{i}\right)+\text { h.c. }
\end{aligned}
$$

(Note that the interactions have been Fierz reordered.) These interactions contribute to pion decay when any of the products $\lambda^{i 1} \lambda^{k 1}$ is nonzero, where $k=1$ for the electron decay channel and $k=2$ for the muon decay channel, and $i$ is the generational index of the unobservable outgoing neutrino. When $i=k$, the contribution interferes 
coherently with the $\mathrm{W}$ exchange amplitude; otherwise, the interference is incoherent. Taking only the real part, we have

$$
\begin{aligned}
& \rho_{k}=\left(\frac{1}{4}\right) \operatorname{Re}\left(\lambda_{L S}^{k 1} \lambda_{R S}^{k 1 *}\right), \rho_{k}^{\prime}=\left(\frac{1}{4}\right) \operatorname{Re}\left(\lambda_{L S}^{i 1} \lambda_{R S}^{k 1 *}\right) \\
& \rho_{k}=\left(\frac{1}{2}\right) \operatorname{Re}\left(\lambda_{L V}^{k 1} \lambda_{R V}^{k 1 *}\right), \quad \rho_{k}^{\prime}=\left(\frac{1}{2}\right) \operatorname{Re}\left(\lambda_{L V}^{i 1} \lambda_{R V}^{k 1 *}\right),
\end{aligned}
$$

for the contributions involving scalar and vector leptoquarks, respectively, where for the $\rho^{\prime}$ we have assumed $i \neq k$. Substituting into equation 3.10, we obtain bounds on products of leptoquark couplings, which are shown in table 3.4.

The Feynman diagrams for the axial-vector leptoquark contributions have the same structure as the Standard Model pion decay diagram, since the effective interactions that lead to these contributions are

$$
\begin{aligned}
\mathcal{L}_{\text {eff }} & \supset \frac{\lambda_{L S_{0}}^{i j} \lambda_{L S_{0}}^{k l *}}{2 m_{S_{0}}^{2}}\left(\bar{d}_{L}^{j, c} \gamma^{\mu} u_{L}^{l, c}\right)\left(\bar{e}_{L}^{k} \gamma_{\mu} \nu_{L}^{i}\right) \\
& -\frac{\lambda_{L S_{1}}^{i j} \lambda_{L S_{1}}^{k l *}}{2 m_{S_{1}}^{2}}\left(\bar{d}_{L}^{j, c} \gamma^{\mu} u_{L}^{l, c}\right)\left(\bar{e}_{L}^{k} \gamma_{\mu} \nu_{L}^{i}\right) \\
& +\frac{\lambda_{L V_{0}}^{i j} \lambda_{L V_{0}}^{k l *}}{m_{V_{0}}^{2}}\left(\bar{u}_{L}^{j} \gamma^{\mu} d_{L}^{l}\right)\left(\bar{e}_{L}^{k} \gamma_{\mu} \nu_{L}^{i}\right) \\
& -\frac{\lambda_{L V_{1}}^{i j} \lambda_{L V_{1}}^{k l *}}{m_{V_{1}}^{2}}\left(\bar{u}_{L}^{j} \gamma^{\mu} d_{L}^{l}\right)\left(\bar{e}_{L}^{k} \gamma_{\mu} \nu_{L}^{i}\right)+\text { h.c. }
\end{aligned}
$$

Since these contributions are chirally suppressed, they will not be bounded as strongly as their pseudoscalar counterparts. They have the effect of changing the ratio of branching ratios to $[15]$

$$
\begin{aligned}
& R_{\pi} \approx R_{\pi}(\mathrm{SM})\left(1+\left(\frac{1}{2}\right) \frac{\left|\lambda_{L S}^{11}\right|^{2}}{\sqrt{2} G_{F} m^{2}}-\left(\frac{1}{2}\right) \frac{\left|\lambda_{L S}^{21}\right|^{2}}{\sqrt{2} G_{F} m^{2}}\right) \\
& R_{\pi} \approx R_{\pi}(\mathrm{SM})\left(1+\frac{\left|\lambda_{L V}^{11}\right|^{2}}{\sqrt{2} G_{F} m^{2}}-\frac{\left|\lambda_{L V}^{21}\right|^{2}}{\sqrt{2} G_{F} m^{2}}\right)
\end{aligned}
$$




\begin{tabular}{|c|c|c|}
\hline Product of couplings & New limit & Previous limit from [15] \\
\hline $\operatorname{Re}\left(\lambda_{L S_{0}}^{11} \lambda_{R S_{0}}^{11 *}\right)$ & $4.6 \times 10^{-7}\left[S_{0}\right]^{2}$ & $1 \times 10^{-6}\left[S_{0}\right]^{2}$ \\
\hline $\operatorname{Re}\left(\lambda_{L S_{0}}^{11} \lambda_{R S_{0}}^{21 *}\right)$ & $2.6 \times 10^{-3}\left[S_{0}\right]^{2}$ & ${ }^{*} 2 \times 10^{-4}\left[S_{0}\right]^{2}$ \\
\hline $\operatorname{Re}\left(\lambda_{L S_{0}}^{21} \lambda_{R S_{0}}^{11 *}\right)$ & $1.3 \times 10^{-5}\left[S_{0}\right]^{2}$ & ${ }^{*} 1 \times 10^{-6}\left[S_{0}\right]^{2}$ \\
\hline $\operatorname{Re}\left(\lambda_{L S_{0}}^{21} \lambda_{R S_{0}}^{21 *}\right)$ & $9.5 \times 10^{-5}\left[S_{0}\right]^{2}$ & $2 \times 10^{-4}\left[S_{0}\right]^{2}$ \\
\hline $\operatorname{Re}\left(\lambda_{L S_{0}}^{31} \lambda_{R S_{0}}^{11 *}\right)$ & $1.3 \times 10^{-5}\left[S_{0}\right]^{2}$ & ${ }^{*} 1 \times 10^{-6}\left[S_{0}\right]^{2}$ \\
\hline $\operatorname{Re}\left(\lambda_{L S_{0}}^{31} \lambda_{R S_{0}}^{21 *}\right)$ & $2.6 \times 10^{-3}\left[S_{0}\right]^{2}$ & ${ }^{*} 2 \times 10^{-4}\left[S_{0}\right]^{2}$ \\
\hline $\operatorname{Re}\left(\lambda_{L S_{1 / 2}}^{11} \lambda_{R S_{1 / 2}}^{11 *}\right)$ & $4.6 \times 10^{-7}\left[S_{1 / 2}\right]^{2}$ & $1 \times 10^{-6}\left[S_{1 / 2}\right]^{2}$ \\
\hline $\operatorname{Re}\left(\lambda_{L S_{1 / 2}}^{11} \lambda_{R S_{1 / 2}}^{21 *}\right)$ & $2.6 \times 10^{-3}\left[S_{1 / 2}\right]^{2}$ & ${ }^{*} 2 \times 10^{-4}\left[S_{1 / 2}\right]^{2}$ \\
\hline $\operatorname{Re}\left(\lambda_{L S_{1 / 2}}^{21} \lambda_{R S_{1 / 2}}^{11 *}\right)$ & $1.3 \times 10^{-5}\left[S_{1 / 2}\right]^{2}$ & ${ }^{*} 1 \times 10^{-6}\left[S_{1 / 2}\right]^{2}$ \\
\hline $\operatorname{Re}\left(\lambda_{L S_{1 / 2}}^{21} \lambda_{R S_{1 / 2}}^{21 *}\right)$ & $9.5 \times 10^{-5}\left[S_{1 / 2}\right]^{2}$ & $2 \times 10^{-4}\left[S_{1 / 2}\right]^{2}$ \\
\hline $\operatorname{Re}\left(\lambda_{L S_{1 / 2}}^{31} \lambda_{R S_{1 / 2}}^{11 *}\right)$ & $1.3 \times 10^{-5}\left[S_{1 / 2}\right]^{2}$ & ${ }^{*} 1 \times 10^{-6}\left[S_{1 / 2}\right]^{2}$ \\
\hline $\operatorname{Re}\left(\lambda_{L S_{1 / 2}}^{31} \lambda_{R S_{1 / 2}}^{21 *}\right)$ & $2.6 \times 10^{-3}\left[S_{1 / 2}\right]^{2}$ & ${ }^{*} 2 \times 10^{-4}\left[S_{1 / 2}\right]^{2}$ \\
\hline $\operatorname{Re}\left(\lambda_{L V_{0}}^{11} \lambda_{R V_{0}}^{11 *}\right)$ & $2.3 \times 10^{-7}\left[V_{0}\right]^{2}$ & $5 \times 10^{-7}\left[V_{0}\right]^{2}$ \\
\hline $\operatorname{Re}\left(\lambda_{L V_{0}}^{11} \lambda_{R V_{0}}^{21 *}\right)$ & $1.3 \times 10^{-3}\left[V_{0}\right]^{2}$ & ${ }^{*} 1 \times 10^{-4}\left[V_{0}\right]^{2}$ \\
\hline $\operatorname{Re}\left(\lambda_{L V_{0}}^{21} \lambda_{R V_{0}}^{11 *}\right)$ & $6.3 \times 10^{-6}\left[V_{0}\right]^{2}$ & ${ }^{*} 5 \times 10^{-7}\left[V_{0}\right]^{2}$ \\
\hline $\operatorname{Re}\left(\lambda_{L V_{0}}^{21} \lambda_{R V_{0}}^{21 *}\right)$ & $4.8 \times 10^{-5}\left[V_{0}\right]^{2}$ & $1 \times 10^{-4}\left[V_{0}\right]^{2}$ \\
\hline $\operatorname{Re}\left(\lambda_{L V_{0}}^{31} \lambda_{R V_{0}}^{11 *}\right)$ & $6.3 \times 10^{-6}\left[V_{0}\right]^{2}$ & ${ }^{*} 5 \times 10^{-7}\left[V_{0}\right]^{2}$ \\
\hline $\operatorname{Re}\left(\lambda_{L V_{0}}^{31} \lambda_{R V_{0}}^{21 *}\right)$ & $1.1 \times 10^{-3}\left[V_{0}\right]^{2}$ & ${ }^{*} 1 \times 10^{-4}\left[V_{0}\right]^{2}$ \\
\hline $\operatorname{Re}\left(\lambda_{L V_{1 / 2}}^{11} \lambda_{R V_{1 / 2}}^{11 *}\right)$ & $2.3 \times 10^{-7}\left[V_{1 / 2}\right]^{2}$ & $5 \times 10^{-7}\left[V_{1 / 2}\right]^{2}$ \\
\hline $\operatorname{Re}\left(\lambda_{L V_{1 / 2}}^{11} \lambda_{R V_{1 / 2}}^{21 *}\right)$ & $1.3 \times 10^{-3}\left[V_{1 / 2}\right]^{2}$ & ${ }^{*} 1 \times 10^{-4}\left[V_{1 / 2}\right]^{2}$ \\
\hline $\operatorname{Re}\left(\lambda_{L V_{1 / 2}}^{21} \lambda_{R V_{1 / 2}}^{11 *}\right)$ & $6.3 \times 10^{-6}\left[V_{1 / 2}\right]^{2}$ & ${ }^{*} 5 \times 10^{-7}\left[V_{1 / 2}\right]^{2}$ \\
\hline $\operatorname{Re}\left(\lambda_{L V_{1 / 2}}^{21} \lambda_{R V_{1 / 2}}^{21 *}\right)$ & $4.8 \times 10^{-5}\left[V_{1 / 2}\right]^{2}$ & $1 \times 10^{-4}\left[V_{1 / 2}\right]^{2}$ \\
\hline $\operatorname{Re}\left(\lambda_{L V_{1 / 2}}^{31} \lambda_{R V_{1 / 2}}^{11 *}\right)$ & $6.3 \times 10^{-6}\left[V_{1 / 2}\right]^{2}$ & ${ }^{*} 5 \times 10^{-7}\left[V_{1 / 2}\right]^{2}$ \\
\hline $\operatorname{Re}\left(\lambda_{L V_{1 / 2}}^{31} \lambda_{R V_{1 / 2}}^{21 *}\right)$ & $1.3 \times 10^{-3}\left[V_{1 / 2}\right]^{2}$ & ${ }^{*} 1 \times 10^{-4}\left[V_{1 / 2}\right]^{2}$ \\
\hline
\end{tabular}

Table 3.4: Updated pseudoscalar leptoquark limits. The starred entries are the previous limits from reference [15] which we believe to be erroneous, as they seem to have been obtained by treating incoherent contributions as being coherent. We use $[l q]$ to represent $\frac{m_{l q}}{100 \mathrm{GeV}}$. 


\begin{tabular}{|c|c|c|}
\hline Product of couplings & New limit & Previous limit from [15] \\
\hline$\left|\lambda_{L S_{0}}^{11}\right|^{2}$ & $1.7 \times 10^{-3}\left[S_{0}\right]^{2}$ & $4 \times 10^{-3}\left[S_{0}\right]^{2}$ \\
$\left|\lambda_{L S_{0}}^{21}\right|^{2}$ & $1.7 \times 10^{-3}\left[S_{0}\right]^{2}$ & $4 \times 10^{-3}\left[S_{0}\right]^{2}$ \\
\hline$\left|\lambda_{L S_{1}}^{11}\right|^{2}$ & $1.7 \times 10^{-3}\left[S_{1}\right]^{2}$ & $4 \times 10^{-3}\left[S_{1}\right]^{2}$ \\
$\left|\lambda_{L S_{1}}^{21}\right|^{2}$ & $1.7 \times 10^{-3}\left[S_{1}\right]^{2}$ & $4 \times 10^{-3}\left[S_{1}\right]^{2}$ \\
\hline$\left|\lambda_{L V_{0}}^{11}\right|^{2}$ & $8.7 \times 10^{-4}\left[V_{0}\right]^{2}$ & $2 \times 10^{-3}\left[V_{0}\right]^{2}$ \\
$\left|\lambda_{L V_{0}}^{21}\right|^{2}$ & $8.7 \times 10^{-4}\left[V_{0}\right]^{2}$ & $2 \times 10^{-3}\left[V_{0}\right]^{2}$ \\
\hline$\left|\lambda_{L V_{1}}^{11}\right|^{2}$ & $8.7 \times 10^{-4}\left[V_{1}\right]^{2}$ & $2 \times 10^{-3}\left[V_{1}\right]^{2}$ \\
$\left|\lambda_{L V_{1}}^{21}\right|^{2}$ & $8.7 \times 10^{-4}\left[V_{1}\right]^{2}$ & $2 \times 10^{-3}\left[V_{1}\right]^{2}$ \\
\hline
\end{tabular}

Table 3.5: Updated axial-vector leptoquark limits. We use $[l q]$ to represent $\frac{m_{l q}}{100 \mathrm{GeV}}$.

Again we limit only one product of couplings at a time, resulting in the bounds that are displayed in table 3.5 .

\subsubsection{Extra Higgs Bosons}

In many extensions of the Standard Model Higgs sector, extra Higgs particles exist whose couplings to fermions are not constrained. We place limits on the couplings of a charged Higgs assuming the Lagrangian term [3]

$$
\frac{g}{2 \sqrt{2} m_{W}} H^{+}\left(K_{q}\left(m_{u}+m_{d}\right) \bar{u} \gamma^{5} d+K_{e} m_{e} \bar{e}\left(1+\gamma^{5}\right) \nu_{e}+K_{\mu} m_{\mu} \bar{\mu}\left(1+\gamma^{5}\right) \nu_{\mu}\right)
$$

where the $K$ couplings are dimensionless. (Although the Higgs may also couple to the scalar bilinear $\bar{u} d$, such an interaction does not affect the decay of the pion, which is pseudoscalar, at tree level.) This leads to

$$
R_{\pi} \approx R_{\pi}(\mathrm{SM})\left(1+2 K_{q}\left(K_{e}-K_{\mu}\right) \frac{m_{\pi}^{2}}{m_{H}^{2}}\right)
$$


Assuming that one product of couplings dominates, we have the limit

$$
K_{q} K_{l}<1.4 \cdot 10^{3}[H]^{2}
$$

In some models, however, the Higgs-lepton couplings are set by the scale of heavier leptons. For example, such couplings arise in some $S U(3) \times U(1)$ models [48]. In particular, if $K_{e}=\frac{m_{\tau}}{m_{e}}$ and $K_{\mu}=\frac{m_{\tau}}{m_{\mu}}$, we obtain the limit

$$
K_{q}<0.42[H]^{2}
$$

\subsubsection{Compositeness}

Models of compositeness induce four-fermion contact interactions, suppressed by two powers of the compositeness scale. In general, these interactions may yield scalar, pseudoscalar, vector, axial-vector, or tensor contributions to Standard Model amplitudes. Ignoring tensor interactions, note that for two Dirac spinors $\psi^{1}$ and $\psi^{2}$,

$$
\begin{aligned}
\bar{\psi}^{1} \psi^{2} & =\bar{\psi}_{L}^{1} \psi_{R}^{2}+\bar{\psi}_{R}^{1} \psi_{L}^{2} \\
\bar{\psi}^{1} \gamma^{5} \psi^{2} & =\bar{\psi}_{L}^{1} \psi_{R}^{2}-\bar{\psi}_{R}^{1} \psi_{L}^{2} \\
\bar{\psi}^{1} \gamma^{\mu} \psi^{2} & =\bar{\psi}_{R}^{1} \gamma^{\mu} \psi_{R}^{2}+\bar{\psi}_{L}^{1} \gamma^{\mu} \psi_{L}^{2} \\
\bar{\psi}^{1} \gamma^{\mu} \gamma^{5} \psi^{2} & =\bar{\psi}_{R}^{1} \gamma^{\mu} \psi_{R}^{2}-\bar{\psi}_{L}^{1} \gamma^{\mu} \psi_{L}^{2}
\end{aligned}
$$

where we have used the definitions for the chiral components of spinors in equation 1.34. In particular, vector and axial-vector interactions couple spinors of the same chirality, while scalar and pseudoscalar interactions "flip" chirality. All gauge bosonfermion couplings in the Standard Model are combinations of vector and axial-vector interactions, so it is often useful to consider chirality-conserving structures, since such contributions interfere with vector boson exchange. The Particle Data Group 
convention for the general form of these interactions is $[36,49]$

$$
\mathcal{L} \supset \frac{g^{2}}{2 \Lambda^{2}}\left(\eta_{L L} \bar{\psi}_{L} \gamma^{\mu} \psi_{L} \bar{\psi}_{L} \gamma_{\mu} \psi_{L}+\eta_{R R} \bar{\psi}_{R} \gamma^{\mu} \psi_{R} \bar{\psi}_{R} \gamma_{\mu} \psi_{R}+2 \eta_{L R} \bar{\psi}_{L} \gamma^{\mu} \psi_{L} \bar{\psi}_{R} \gamma_{\mu} \psi_{R}\right)
$$

where $g$ is an arbitrary coupling, and the $\psi$ are fermionic fields that may be either leptons or quarks. Usually, conservation of baryon number and lepton number is assumed, so that only operators with four leptons, four quarks, or two leptons and two quarks are considered [36]. Following convention, we will choose $\frac{g^{2}}{4 \pi}=1$ and set each $\eta$ to either 0 or \pm 1 , then extract a limit on $\Lambda$.

We consider the effect of the operator

$$
\frac{g^{2}}{2 \Lambda^{2}}\left(\bar{u}_{L} \gamma^{\mu} d_{L}\right)\left(\bar{e}_{L} \gamma^{\mu}\left(\nu_{e}\right)_{L}\right)
$$

with $\frac{g^{2}}{4 \pi}=1$ chosen as described above, and limit the compositeness scale $\Lambda$. Although its contribution is chirally suppressed (as expected, since the operator conserves chirality), the contact operator interferes coherently with the Standard Model contribution to pion decay. It changes the ratio of branching ratios to

$$
R_{\pi} \approx R_{\pi}(\mathrm{SM})\left(1+\frac{\sqrt{2} \pi}{G_{F} \Lambda^{2}}\right)
$$

leading to the bound

$$
\Lambda>8.5 \mathrm{TeV}
$$

In the massless fermion limit, only vector and axial-vector operators, not scalar and pseudoscalar operators, can interfere with vector boson exchange, since $\psi_{L}$ and $\psi_{R}$ decouple from each other as $m \rightarrow 0$. Now, although the massless limit is a good approximation at high-energy colliders, pion decay requires a chirality flip (and hence a mass term), and so (pseudo)scalar operators may be tightly constrained using the 
pion branching ratios. We thus place limits on the pseudoscalar interactions

$$
\frac{g^{2}}{2 \Lambda^{2}}\left(\bar{u} \gamma^{5} d\right)\left(\bar{e} \gamma^{5} \nu\right)
$$

again assuming $\frac{g^{2}}{4 \pi}=1$, and neglecting the analogous operator which contributes to the muon decay mode. For the operator involving the electron neutrino, the effective strength of the coupling is

$$
\frac{\rho_{e}}{\Lambda^{2}}=\frac{\pi}{\Lambda^{2}}
$$

and so we obtain a limit of

$$
\Lambda>5.2 \cdot 10^{2} \mathrm{TeV}
$$

For the operators involving the muon and tau neutrinos, we have

$$
\frac{\rho_{e}^{\prime}}{\Lambda^{2}}=\frac{\pi}{\Lambda^{2}}
$$

and the limit is

$$
\Lambda>1.0 \cdot 10^{2} \mathrm{TeV}
$$

As expected, these limits are much better than that on the vector operator above, because pseudoscalar contributions to pion decay are not chirally suppressed.

Finally, we limit the scalar contact interactions

$$
\frac{g^{2}}{2 \Lambda^{2}}(\bar{u} d)(\bar{e} \nu)
$$

which are also not chirally suppressed. Although these operators do not contribute to pion decay at tree level, because the pion is a pseudoscalar particle, renormalization leads to a modification of $R_{\pi}$ at one loop. We may see this phenomenon explicitly by 
working in the quark chiral basis, writing

$$
\frac{g^{2}}{2 \Lambda^{2}}(\bar{u} d)(\bar{e} \nu)=\frac{g^{2}}{2 \Lambda^{2}}\left(\bar{u}_{R} d_{L}\right)\left(\bar{e}_{R} \nu_{L}\right)+\frac{g^{2}}{2 \Lambda^{2}}\left(\bar{u}_{L} d_{R}\right)\left(\bar{e}_{R} \nu_{L}\right)
$$

where we have ignored right-handed neutrinos. The two chiral operators above are renormalized differently, so if they start out with equal coefficients at the scale $\Lambda$, then their coefficients will be different at the weak scale, where the renormalization group equations change as we integrate out the $W$. The difference between the coefficients corresponds to a pseudoscalar operator, which may then be limited as before. We write the operators

$$
\begin{aligned}
& \mathcal{O}_{1}=c_{1}\left(\bar{e}_{R} L_{L}\right)\left(\bar{Q}_{L} d_{R}\right) \\
& \mathcal{O}_{2}=c_{2}\left(\bar{e}_{R} L_{L}\right)\left(\bar{u}_{R} Q_{L}\right) \\
& \mathcal{O}_{3}=c_{3}\left(-\frac{1}{8}\right)\left(\bar{e}_{R} \sigma^{\mu \nu} L_{L}\right)\left(\bar{u}_{R} \sigma_{\mu \nu} Q_{L}\right),
\end{aligned}
$$

where we have suppressed $S U(2)$ indices and taken $\sigma^{\mu \nu}=\frac{i}{2}\left[\gamma^{\mu}, \gamma^{\nu}\right]$. The coefficients satisfy the partially coupled renormalization group equations

$$
M \frac{\partial c_{i}}{\partial M}=\frac{1}{32 \pi^{2}} \gamma^{i j} c_{j}
$$

where summation over repeated indices is implied and the anomalous dimension matrix is [33]

$$
\gamma=\left(\begin{array}{ccc}
6 g^{2}+\frac{98}{9} g^{\prime 2} & 0 & 0 \\
0 & 6 g^{2}+\frac{128}{9} g^{\prime 2} & 6 g^{2}+10 g^{\prime 2} \\
0 & \frac{9}{2} g^{2}+\frac{15}{2} g^{\prime 2} & 12 g^{2}+\frac{103}{9} g^{\prime 2}
\end{array}\right)
$$

In this expression, $g$ and $g^{\prime}$ are the $S U(2)$ and $U(1)$ couplings, respectively, and are 
renormalized according to [39]

$$
\begin{aligned}
& M \frac{\partial g}{\partial M}=\frac{1}{32 \pi^{2}}\left(-\frac{19}{3}\right) g^{3} \\
& M \frac{\partial g^{\prime}}{\partial M}=\frac{1}{32 \pi^{2}}\left(\frac{41}{5}\right) g^{\prime 3}
\end{aligned}
$$

Starting with $c_{1}=c_{2}=\frac{2 \pi}{\Lambda^{2}}, c_{3}=0$ at $M=1 \mathrm{TeV}$, numerical integration yields $\left|c_{2}-c_{1}\right| \approx\left(2.5 \cdot 10^{-3}\right) \frac{2 \pi}{\Lambda^{2}}$ at $M=100 \mathrm{GeV}$, which is approximately the weak scale. We thus effectively have

$$
\begin{aligned}
& \frac{\rho_{e}}{\Lambda^{2}}=\left(2.5 \cdot 10^{-3}\right) \frac{\pi}{\Lambda^{2}}, \\
& \frac{\rho_{e}^{\prime}}{\Lambda^{2}}=\left(2.5 \cdot 10^{-3}\right) \frac{\pi}{\Lambda^{2}},
\end{aligned}
$$

which lead to the limits

$$
\begin{aligned}
& \Lambda>26 \mathrm{TeV} \text { (electron neutrino), } \\
& \Lambda>5.0 \mathrm{TeV} \text { (other neutrinos) }
\end{aligned}
$$

These limits are weaker than those in the pseudoscalar case, because although the scalar contributions to pion decay are not chirally suppressed, they involve one loop diagrams (through the renormalization group equations), which are suppressed by a power of the gauge coupling relative to their tree level counterparts.

\subsubsection{Supercompositeness}

Supercomposite models treat (s)quarks and (s)leptons as composite particles, with the compositeness scale above the scale of supersymmetry breaking so that the induced contact operators include supersymmetric particles. The leading contributions of such models to pion decay come from dimension-five operators in the Lagrangian. 


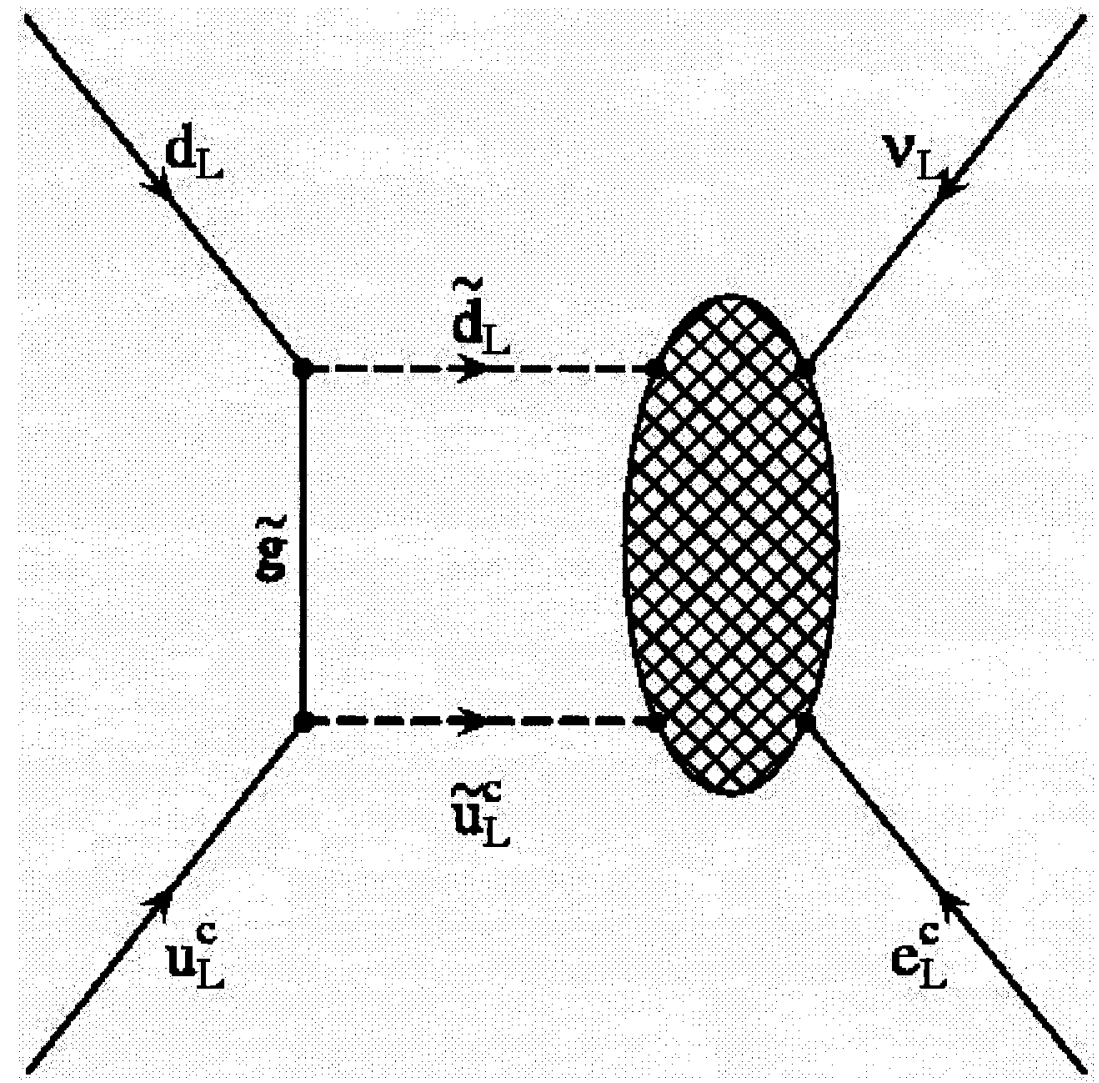

Figure 3.3: A contribution to pion decay involving gluino dressing of a dimensionfive supercompositeness operator. 
(The operators are dimension-five because they involve two scalars and two fermions, as opposed to the dimension-six compositeness operators of the previous section, which involved four fermions.) These operators are suppressed by one power of the supercompositeness scale, and come from two types of terms in the Lagrangian. The F-terms come from the superpotential, as discussed in section 3.3.1, while the Dterms are the Lagrangian terms that can not be obtained from the superpotential, because they involve the complex conjugates of superfields. The terms which respect the $S U(3) \times S U(2) \times U(1)$ gauge symmetries and R-parity are [50]

$$
\begin{aligned}
& \left(L_{L} E_{L}^{c} H_{u}^{*}\right)_{D},\left(Q_{L} D_{L}^{c} H_{u}^{*}\right)_{D},\left(Q_{L} U_{L}^{c} H_{d}^{*}\right)_{D},\left(L_{L} L_{L} H_{u} H_{u}\right)_{F} \\
& \left(Q_{L} Q_{L} U_{L}^{c} D_{L}^{c}\right)_{F},\left(Q_{L} U_{L}^{c} L_{L} E_{L}^{c}\right)_{F},\left(Q_{L} Q_{L} Q_{L} L_{L}\right)_{F},\left(U_{L}^{c} U_{L}^{c} D_{L}^{c} E_{L}^{c}\right)_{F}
\end{aligned}
$$

The contributions to pion decay from the D-terms involve the couplings of Higgs particles to first-generation quarks and leptons, which are small, and so we only consider the F-term contributions to pion decay. Examining the above operators, we see that there is only one F-term that contributes to pion decay, $\frac{1}{M} Q_{L} U_{L}^{c} L_{L} E_{L}^{c}$, where $M$ is the supercompositeness scale. We may calculate its contribution by dressing dimension-five compositeness operators [51]. The dominant contribution to the amplitude will be from gluino exchange, shown in figure 3.3. After performing the loop integral (see the calculation in appendix D), taking $m_{g} \sim m_{\tilde{u}} \sim m_{\tilde{d}}$, we find that the amplitude associated with this diagram is

$$
\mathcal{M}=\frac{\alpha_{s}}{2 \sqrt{6} \pi} \frac{1}{M m_{g}}\left[\bar{l}\left(1-\gamma^{5}\right) \nu_{l}\right] \tilde{f}_{\pi}
$$

up to a complex phase, where $M$ is the supercompositeness scale and $m_{g}$ is the gluino mass. Neglecting the effect of the contribution to the muon decay channel, we may 
equate

$$
\frac{\rho_{e}}{\Lambda^{2}}=\frac{\alpha_{s}}{2 \sqrt{6} \pi} \frac{1}{M m_{g}}
$$

For $\alpha_{s} \sim 0.1$, this leads to the limit

$$
M m_{g}>6 \cdot 10^{2} \mathrm{TeV}^{2}
$$

Taking $m_{g} \sim 1 \mathrm{TeV}$ for illustration, we are left with a supercompositeness bound of

$$
M>6 \cdot 10^{2} \mathrm{TeV}
$$




\section{References}

[1] David J. Griffiths. Introduction to elementary particles. New York, USA: Wiley (1987) $392 \mathrm{p}$.

[2] H. E. Haber, Gordon L. Kane, and T. Sterling. The fermion mass scale and possible effects of Higgs bosons on experimental observables. Nucl. Phys., B161:493, 1979.

[3] John F. Donoghue and Ling Fong Li. Properties of charged Higgs bosons. Phys. Rev., D19:945, 1979.

[4] Bruce McWilliams and Ling-Fong Li. Virtual effects of Higgs particles. Nucl. Phys., B179:62, 1981.

[5] Robert E. Shrock. General theory of weak leptonic and semileptonic decays. 1. Leptonic pseudoscalar meson decays, with associated tests for, and bounds on, neutrino masses and lepton mixing. Phys. Rev., D24:1232, 1981.

[6] Oruganti U. Shanker. Pi lepton 2, K lepton 3 and K0 - anti-K0 constraints on leptoquarks and supersymmetric particles. Nucl. Phys., B204:375, 1982.

[7] K. Mursula, M. Roos, and F. Scheck. The Lorentz structure of leptonic charged weak interactions. 1. Nucl. Phys., B219:321, 1983.

[8] Lawrence J. Hall and Mahiko Suzuki. Explicit R-parity breaking in supersymmetric models. Nucl. Phys., B231:419, 1984.

[9] W. Buchmuller and D. Wyler. Constraints on SU(5) type leptoquarks. Phys. Lett., B177:377, 1986.

[10] Yoshiki Kizukuri. Effects of lepto-quarks on pi to lepton neutrino, mu to electron gamma, and electric dipole moment of the electron in $\mathrm{E}(6)$ models based on superstring. Phys. Lett., B185:183, 1987. 
[11] W. Buchmuller, R. Ruckl, and D. Wyler. Leptoquarks in lepton quark collisions. Phys. Lett., B191:442-448, 1987.

[12] B. A. Campbell, John R. Ellis, K. Enqvist, M. K. Gaillard, and Dimitri V. Nanopoulos. Superstring models challenged by rare processes. Int. J. Mod. Phys., A2:831, 1987.

[13] Vernon D. Barger, G. F. Giudice, and Tao Han. Some new aspects of supersymmetry R-parity violating interactions. Phys. Rev., D40:2987, 1989.

[14] Miriam Leurer. A comprehensive study of leptoquark bounds. Phys. Rev., D49:333-342, 1994.

[15] Sacha Davidson, David C. Bailey, and Bruce A. Campbell. Model independent constraints on leptoquarks from rare processes. Z. Phys., C61:613-644, 1994.

[16] P. Herczeg. A note on limits on new interactions from the $(\pi \rightarrow e \nu) /(\pi \rightarrow \mu \nu)$ branching ratio. Phys. Rev., D52:3949-3957, 1995.

[17] Herbert K. Dreiner. An introduction to explicit R-parity violation. 1997. hep$\mathrm{ph} / 9707435$.

[18] Gautam Bhattacharyya. A brief review of R-parity-violating couplings. 1997. hep-ph/9709395.

[19] B. C. Allanach, A. Dedes, and Herbert K. Dreiner. Bounds on R-parity violating couplings at the weak scale and at the GUT scale. Phys. Rev., D60:075014, 1999.

[20] Herbert K. Dreiner, Giacomo Polesello, and Marc Thormeier. Bounds on broken R-parity from leptonic meson decays. Phys. Rev., D65:115006, 2002.

[21] Herbi Dreiner, Giacomo Polesello, and Marc Thormeier. Bounds on broken Rparity from NOMAD and CHORUS. 2002. hep-ph/0207160.

[22] Margarete Herz. Bounds on leptoquark and supersymmetric, R-parity violating interactions from meson decays. (In German). 2002. hep-ph/0301079.

[23] Marc Chemtob. Phenomenological constraints on broken R parity symmetry in supersymmetry models. Prog. Part. Nucl. Phys., 54:71-191, 2005.

[24] R. Barbier et al. R-parity violating supersymmetry. Phys. Rept., 420:1-202, 2005. 
[25] Jens Erler and Michael J. Ramsey-Musolf. Low energy tests of the weak interaction. Prog. Part. Nucl. Phys., 54:351-442, 2005.

[26] H. K. Dreiner, M. Kramer, and Ben O'Leary. Bounds on R-parity violation from leptonic and semi- leptonic meson decays. Phys. Rev., D75:114016, 2007.

[27] Michael J. Ramsey-Musolf, Shufang Su, and Sean Tulin. Pion leptonic decays and supersymmetry. Phys. Rev., D76:095017, 2007.

[28] M. J. Ramsey-Musolf and S. Su. Low energy precision test of supersymmetry. Phys. Rept., 456:1-88, 2008.

[29] A. Masiero, P. Paradisi, and R. Petronzio. Anatomy and phenomenology of the lepton flavor universality in SUSY theories. 2008. 0807.4721.

[30] P. Herczeg. Beta decay beyond the Standard Model. Prog. Part. Nucl. Phys., 46:413-457, 2001.

[31] TRIUMF. Pienu experiment (e1072).

[32] PSI. Pen experiment (r-05-01).

[33] Bruce A. Campbell and David W. Maybury. Constraints on scalar couplings From $\pi^{ \pm} \rightarrow l^{ \pm} \nu_{l}$. Nucl. Phys., B709:419-439, 2005.

[34] William J. Marciano and A. Sirlin. Radiative corrections to pi(lepton 2) decays. Phys. Rev. Lett., 71:3629-3632, 1993.

[35] Vincenzo Cirigliano and Ignasi Rosell. $\pi / K \rightarrow e \nu$ branching ratios to $O\left(e^{2} p^{4}\right)$ in chiral perturbation theory. JHEP, 10:005, 2007.

[36] W.-M. Yao et al. Review of particle physics. J. Phys., G33:1-1232, 2006.

[37] Steven Weinberg. Phenomenological Lagrangians. Physica, A96:327, 1979.

[38] H. Georgi. Weak interactions and modern particle theory. Menlo Park, USA: Benjamin/Cummings (1984) $165 \mathrm{p}$.

[39] Pierre Ramond. Journeys beyond the Standard Model. Reading, Mass., Perseus Books, 1999.

[40] J. Wess and J. Bagger. Supersymmetry and supergravity. Princeton, USA: Univ. Pr. (1992) 259 p.

[41] Stephen P. Martin. A supersymmetry primer. 1997. hep-ph/9709356. 
[42] Alexei Yu. Smirnov and Francesco Vissani. Upper bound on all products of Rparity violating couplings $\lambda^{\prime}$ and $\lambda^{\prime \prime}$ from proton decay. Phys. Lett., B380:317323, 1996.

[43] Carl E. Carlson, Probir Roy, and Marc Sher. New bounds on R-parity violating couplings. Phys. Lett., B357:99-104, 1995.

[44] Graham G. Ross. Grand unified theories. Reading, USA: Benjamin/Cummings (1984) 497 p. (Frontiers In Physics, 60).

[45] Jogesh C. Pati and Abdus Salam. Lepton number as the fourth color. Phys. Rev., D10:275-289, 1974.

[46] H. Georgi and S. L. Glashow. Unity of all elementary particle forces. Phys. Rev. Lett., 32:438-441, 1974.

[47] Edward Farhi and Leonard Susskind. Technicolor. Phys. Rept., 74:277, 1981.

[48] Jihn E. Kim and G. Segre. Possible signals for Higgs meson production in highenergy experiments. Phys. Lett., B78:75, 1978.

[49] E. Eichten, Kenneth D. Lane, and Michael Edward Peskin. New tests for quark and lepton substructure. Phys. Rev. Lett., 50:811-814, 1983.

[50] Steven Weinberg. Supersymmetry at ordinary energies. 1. Masses and conservation laws. Phys. Rev., D26:287, 1982.

[51] B. A. Campbell and K. A. Peterson. Tests of supercomposite models in pion decay and muon conversion. Phys. Lett., B192:401-405, 1987. 


\section{Chapter 4}

\section{Conclusion}

The Standard Model accurately predicts many experimental phenomena, but is thought to be incomplete. However, there are many different theories of physics beyond the Standard Model. In order to decide which one provides the best description of nature, we must determine the validity of these theories through experiment.

The ratio $R_{\pi}$ is a highly sensitive test of new physics. Using the agreement between the experimental value of $R_{\pi}$ and its theoretical prediction, we have updated existing limits and placed new ones on proposed pseudoscalar, scalar and axial-vector interactions, using an effective field theory framework. We have also performed the computation of the leading QCD correction to pion decay, enabling us to strengthen all of our bounds by a factor of 2 .

Currently, the experimental value of $R_{\pi}$ is more uncertain than its theoretical counterpart by a factor of 40 . Proposed experiments at TRIUMF [1] and PSI [2] intend to reduce the experimental uncertainty to $0.1 \%$ and $0.05 \%$, respectively, which would correspond to an improvement of approximately one order of magnitude. The resulting data should thus considerably strengthen the bounds we have obtained. 


\section{References}

[1] TRIUMF. Pienu experiment (e1072).

[2] PSI. Pen experiment (r-05-01). 


\section{Appendix A}

\section{Dimensional Regularization Reference Formulae}

Divergent integrals in amplitudes, coming from loop diagrams, are frequently evaluted using dimensional regularization. We list some results here that are derived in Ramond [1]. Note that these formulae are for integration over $d$-dimensional $E u$ clidean spacetime; a Wick rotation is usually performed on the ordinary Minkowski spacetime integral to bring it into the appropriate form. As well, if the denominator in an integral is $\left(l^{2}+2 l \cdot p+\Delta\right)^{A}$, for some (real) external momentum $p$, we have completed the square below by substituting $l \rightarrow l+p$, so that $\Delta \rightarrow \Delta-p^{2}$.

$$
\begin{gathered}
\int \frac{d^{d} l}{(2 \pi)^{d}} \frac{1}{\left(l^{2}+\Delta\right)^{A}}=\frac{\Gamma\left(A-\frac{d}{2}\right)}{(4 \pi)^{\frac{d}{2}} \Gamma(A)} \frac{1}{\left(M^{2}\right)^{A-\frac{d}{2}}} \\
\int \frac{d^{d} l}{(2 \pi)^{d}} \frac{l_{\mu}}{\left(l^{2}+\Delta\right)^{A}}=0 \\
\int \frac{d^{d} l}{(2 \pi)^{d}} \frac{l_{\mu} l_{\nu}}{\left(l^{2}+\Delta\right)^{A}}=\frac{\Gamma\left(A-1-\frac{d}{2}\right)}{(4 \pi)^{\frac{d}{2}} \Gamma(A)}\left(\frac{\delta_{\mu \nu}}{2}\right) \frac{1}{\left(M^{2}\right)^{A-1-\frac{d}{2}}}
\end{gathered}
$$




$$
\begin{gathered}
\int \frac{d^{d} l}{(2 \pi)^{d}} \frac{l_{\mu} l_{\nu} l_{\rho}}{\left(l^{2}+\Delta\right)^{A}}=0 \\
\int \frac{d^{d} l}{(2 \pi)^{d}} \frac{l_{\mu} l_{\nu} l_{\rho} l_{\sigma}}{\left(l^{2}+\Delta\right)^{A}}=\frac{\Gamma\left(A-2-\frac{d}{2}\right)}{(4 \pi)^{\frac{d}{2}} \Gamma(A)}\left(\frac{\delta_{\mu \nu} \delta_{\rho \sigma}+\delta_{\mu \rho} \delta_{\nu \sigma}+\delta_{\mu \sigma} \delta_{\nu \rho}}{4}\right) \frac{1}{\left(M^{2}\right)^{A-2-\frac{d}{2}}}
\end{gathered}
$$

In the above, $\Delta$ is assumed to be some real Lorentz invariant quantity that does not depend on $l$, although it may involve any external momenta. Although $A$ is usually a positive integer, it may in principle be any complex number such that the arguments of the gamma functions, if they are integral, only take positive values. Note that from the form of the first integral, the structure of the remaining integrals is determined by symmetry and Lorentz invariance. In general, if there is an odd number of $l_{\mu}$ in the numerator, the integral will vanish by symmetry. 


\section{References}

[1] Pierre Ramond. Field theory: a modern primer. Front. Phys., 74:1-329, 1989. 


\section{Appendix B}

\section{Derivation of Pion Decay Operator Anomalous Dimension}

We compute the anomalous dimension $\gamma$ of the operator $\mathcal{O}=\left[\bar{e}\left(1-\gamma_{5}\right) \nu_{e}\right]\left[\bar{u} \gamma_{5} d\right]$, to obtain the QCD correction to the limit in equation 3.4. Since the operator involves two quark fields and no gluons, equation 2.35 tells us that

$$
\gamma=M \frac{\partial}{\partial M}\left(-\delta_{\mathcal{O}}+\delta_{2}\right)
$$

where $M$ is the renormalization scale, $\delta_{2}$ is the counterterm that cancels the quark field strength divergences, and $\delta_{\mathcal{O}}$ is the counterterm that cancels the divergences in the QCD corrections to $\mathcal{O}$.

The computation of $\delta_{2}$ through dimensional regularization is a standard result from quantum field theory (see [1]), and gives

$$
\delta_{2}=-\frac{4}{3} \frac{g_{s}^{2}}{(4 \pi)^{2}} \frac{\Gamma\left(2-\frac{d}{2}\right)}{\left(M^{2}\right)^{2-\frac{d}{2}}}
$$

where $d$ is the number of dimensions and $g_{s}$ is the strong coupling constant. It remains for us to calculate $\delta_{\mathcal{O}}$, using the diagrams that provide QCD corrections to $\mathcal{O}$.

For our operator renormalization condition, we require that at $s=t=u=$ 


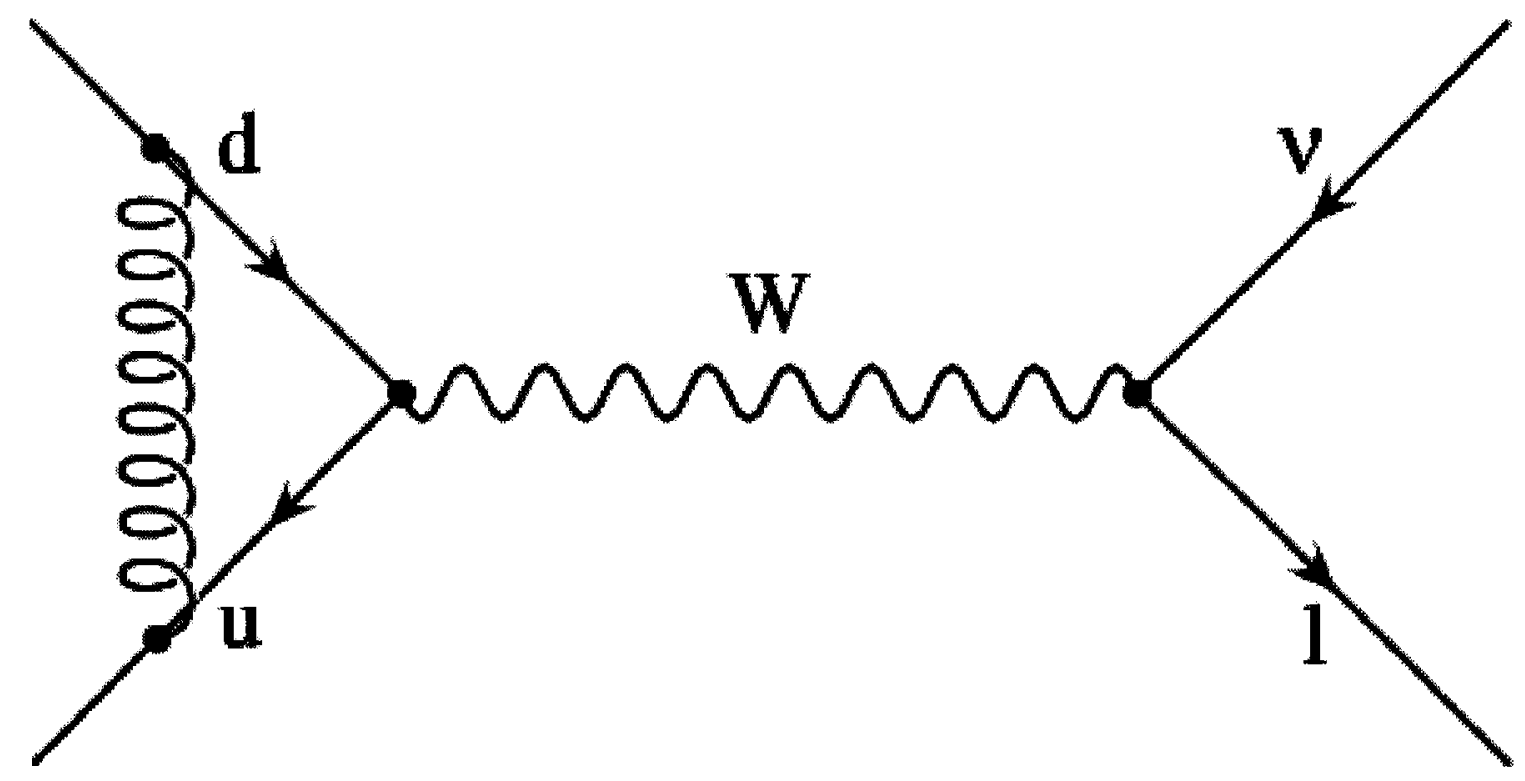

Figure B.1: The lowest order QCD correction to the operator $\left[\bar{e}\left(1-\gamma_{5}\right) \nu_{e}\right]\left[\bar{u} \gamma_{5} d\right]$.

$-M^{2}$, the coefficient of the four-fermion operator $\left[\bar{e}\left(1-\gamma_{5}\right) \nu_{e}\right]\left[\bar{u} \gamma_{5} d\right]$ is 1 . At one loop, there is only one QCD correction to $\mathcal{O}$, shown in figure B.1. To preserve the renormalization condition, we will need to adjust the counterterm $\delta_{\mathcal{O}}$ to exactly cancel out this correction. Since the two quark propagators each give $\frac{1}{k}$ and the gluon propagator gives $\frac{1}{k^{2}}$, where $k$ is the loop momentum, we expect the diagram in figure B.1 to be logarithmically divergent. Before explicitly computing the divergence, we expand the quark part of the operator in terms of chiral spinors, yielding

$$
\left[\bar{e}\left(1-\gamma_{5}\right) \nu_{e}\right]\left[\bar{u} \gamma_{5} d\right]=\left[\bar{e}\left(1-\gamma_{5}\right) \nu_{e}\right]\left[\bar{u}_{L} d_{R}-\bar{u}_{R} d_{L}\right]
$$

To simplify the $\gamma$ matrix algebra, we will work in the chiral basis, computing only the part of the amplitude that is proportional to $\bar{u}_{L} d_{R}$. As we will see, the other half 
of the amplitude is identical. Using the Feynman rules, we get

$$
\mathcal{M}=\left[\bar{e}\left(1-\gamma_{5}\right) \nu_{e}\right]\left[\bar{u}_{L} \int \frac{d^{4} k}{(2 \pi)^{4}}\left(i g_{s} T^{a} \gamma^{\mu}\right) \frac{i(\not p-\not k)}{(p-k)^{2}}\left(\frac{-i}{k^{2}}\right) \frac{i(\not p-\not k)}{(p-k)^{2}}\left(i g_{s} T^{a} \gamma_{\mu}\right) d_{R}\right]
$$

where $p$ is the external quark momentum, the $T^{a}$ are the Gell-Mann matrices defined in equation 1.45 , and summation over $a$ is implied. In the above, we have used the slash notation $\not p=p_{\mu} \gamma^{\mu}$.

Now, none of the quantities between the two Gell-Mann matrices carry $S U(3)$ indices, so we may immediately use the relation

$$
T^{a} T^{a}=\frac{4}{3} I
$$

where $I$ is the $3 \times 3$ identity matrix in $S U(3)$ space. Since we are only interested in the divergence of $\mathcal{M}$, which occurs as $k \rightarrow \infty$, we may neglect $p$, obtaining

$$
\mathcal{M}=\left[\bar{e}\left(1-\gamma_{5}\right) \nu_{e}\right]\left[\bar{u}_{L} \int \frac{d^{4} k}{(2 \pi)^{4}}\left(-\frac{4}{3} i g_{s}^{2}\right)\left(\frac{k^{2} \gamma^{\mu} \gamma_{\mu}}{\left(k^{2}-\Delta\right)^{3}}\right) d_{R}\right]
$$

where $\Delta$ is some function of invariants such as the quark masses and external momenta. Since we only need the divergent part of $\mathcal{M}$ to get $\gamma_{\mathcal{O}}$, it suffices to take $\Delta=M^{2}$; any renormalization schemes that choose the invariants to be $\mathcal{O}\left(M^{2}\right)$ will yield the same results for divergences, although the finite parts of amplitudes may differ [1].

We evaluate the integral using dimensional regularization. The expression $\gamma^{\mu} \gamma_{\mu}$ must be adjusted in $d$ dimensions, as the number of $\gamma$ matrices in a theory depends on the number of dimensions. Omitting the proof, we write $\gamma^{\mu} \gamma_{\mu}=d$. Note that this expression reduces to the expected result when $d=4$. In $d$ dimensions, then, the 
amplitude is

$$
\begin{aligned}
\mathcal{M} & =\left(\frac{4}{3} g_{s}^{2}\right)\left[\bar{e}\left(1-\gamma_{5}\right) \nu_{e}\right]\left[\bar{u}_{L} \frac{1}{(4 \pi)^{\frac{d}{2}}} \frac{d^{2}}{4} \Gamma\left(2-\frac{d}{2}\right)\left(\frac{1}{\Delta}\right)^{2-\frac{d}{2}} d_{R}\right]+\text { finite } \\
& \rightarrow\left(\frac{16}{3} g_{s}^{2}\right)\left[\bar{e}\left(1-\gamma_{5}\right) \nu_{e}\right]\left[\bar{u}_{L} \frac{\Gamma\left(2-\frac{d}{2}\right)}{(4 \pi)^{2}}\left(\frac{1}{M^{2}}\right)^{2-\frac{d}{2}} d_{R}\right]
\end{aligned}
$$

where we have taken $d \rightarrow 4$ and $\Delta=M^{2}$ after evaluating the integral using a standard formula (see appendix A), keeping only the divergent piece. None of this analysis depended on the chirality of the quarks, and so the part of the amplitude proportional to $\bar{u}_{R} d_{L}$ can be obtained by the substitution $L \leftrightarrow R$.

To cancel the divergent amplitude from figure B.1, it follows that we must take the counterterm

$$
\mathcal{L} \supset \delta_{\mathcal{O}}\left[\bar{e}\left(1-\gamma_{5}\right) \nu_{e}\right]\left[\bar{u} \gamma_{5} d\right]
$$

where we have computed the coefficient to be

$$
\delta_{\mathcal{O}}=-\frac{16}{3} \frac{g_{s}^{2}}{(4 \pi)^{2}} \frac{\Gamma\left(2-\frac{d}{2}\right)}{\left(M^{2}\right)^{2-\frac{d}{2}}}
$$

Using this result with the quark field strength counterterm, we find that the anomalous dimension of the pseudoscalar pion decay operator is

$$
\gamma=-8 \frac{g_{s}^{2}}{(4 \pi)^{2}}=-2 \frac{\alpha_{s}}{\pi}
$$




\section{References}

[1] Michael Edward Peskin and Daniel V. Schroeder. An introduction to quantum field theory. Reading, USA: Addison-Wesley (1995) $842 \mathrm{p}$. 


\section{Appendix C}

\section{Fierz Transformations}

We use the spinor indices of Wess and Bagger [1], where a two-component Weyl spinor is written as $\psi^{\alpha}$, and its complex conjugate is given by the dotted spinor

$$
\bar{\psi}^{\dot{\alpha}}=\left(\psi^{\alpha}\right)^{*}
$$

Indices may be raised and lowered by the matrices

$$
\begin{gathered}
\epsilon_{\alpha \beta}=\epsilon_{\dot{\alpha} \dot{\beta}}=i \sigma^{2}, \\
\epsilon^{\alpha \beta}=\epsilon^{\dot{\alpha} \dot{\beta}}=-i \sigma^{2} .
\end{gathered}
$$

A Lorentz scalar may be formed by the appropriate combination of two spinors, or their conjugates:

$$
\begin{aligned}
& \psi \chi=\psi^{\alpha} \chi_{\alpha} \\
& \bar{\psi} \bar{\chi}=\bar{\psi}_{\dot{\alpha}} \bar{\chi}^{\dot{\alpha}}
\end{aligned}
$$

Now, the basic Fierz identity is [2]

$$
\delta_{\alpha \beta} \delta_{\gamma \delta}=\frac{1}{2}\left(\delta_{\alpha \delta} \delta_{\gamma \beta}+\sigma_{\alpha \delta}^{i} \sigma_{\gamma \beta}^{i}\right)
$$


where the last term is summed over the Pauli matrices, $1 \leq i \leq 3$. By manipulating this identity, we may rearrange the indices on many products of fermion bilinears. For instance, it trivially follows that

$$
\delta_{\alpha}^{\beta} \delta_{\dot{\beta}}^{\dot{\alpha}}=\frac{1}{2}\left(\sigma^{\mu}\right)_{\alpha \dot{\beta}}\left(\bar{\sigma}_{\mu}\right)^{\dot{\alpha} \beta}
$$

and so we have

$$
\begin{aligned}
(\psi \chi)(\bar{\theta} \bar{\eta}) & =\left(\psi^{\alpha} \chi_{\alpha}\right)\left(\bar{\theta}_{\dot{\alpha}} \bar{\eta}^{\dot{\alpha}}\right) \\
& =\left(\psi^{\alpha} \delta_{\alpha}^{\beta} \chi_{\beta}\right)\left(\bar{\theta}_{\dot{\alpha}} \delta_{\dot{\beta}}^{\dot{\alpha}} \bar{\eta}^{\dot{\beta}}\right) \\
& =\frac{1}{2} \psi^{\alpha}\left(\sigma^{\mu}\right)_{\alpha \dot{\beta}} \chi_{\beta} \bar{\theta}_{\dot{\alpha}}\left(\bar{\sigma}_{\mu}\right)^{\dot{\alpha} \beta} \bar{\eta}^{\dot{\beta}} \\
& =-\frac{1}{2}\left(\psi^{\alpha}\left(\sigma^{\mu}\right)_{\alpha \dot{\beta}} \bar{\eta}^{\dot{\beta}}\right)\left(\bar{\theta}_{\dot{\alpha}}\left(\bar{\sigma}_{\mu}\right)^{\dot{\alpha} \beta} \chi_{\beta}\right) .
\end{aligned}
$$

(Note that the minus sign in the last line comes from the anticommutation of the spinors.) Replacing the arbitrary spinors above by those involved in figure 3.2, we get

$$
\left(\bar{e}_{L}^{c} u_{L}\right)\left(\bar{d}_{L} \nu_{L}^{c}\right)=-\frac{1}{2}\left(\bar{e}_{L}^{c} \sigma^{\mu} \nu_{L}^{c}\right)\left(\bar{d}_{L} \bar{\sigma}_{\mu} u_{L}\right)
$$

In four-component notation, this corresponds to a $V-A$ (vector minus axial-vector) interaction, the same type as the weak interaction in the Standard Model.

We list some useful Fierz identities below, suppressing spinor indices. Their derivations are similar to the one we have just performed, and involve the tensors

$$
\begin{gathered}
\sigma^{\mu \nu}=\frac{1}{4}\left(\sigma^{\mu} \bar{\sigma}^{\nu}-\sigma^{\nu} \bar{\sigma}^{\mu}\right), \\
\bar{\sigma}^{\mu \nu}=\frac{1}{4}\left(\bar{\sigma}^{\mu} \sigma^{\nu}-\bar{\sigma}^{\nu} \sigma^{\mu}\right) . \\
(\psi \chi)(\theta \eta)=-\frac{1}{2}\left[(\psi \eta)(\theta \chi)-\left(\psi \sigma^{\mu \nu} \eta\right)\left(\theta \sigma_{\mu \nu} \chi\right)\right]
\end{gathered}
$$




$$
\begin{gathered}
(\psi \chi)(\bar{\theta} \bar{\eta})=-\frac{1}{2}\left(\psi \sigma^{\mu} \bar{\eta}\right)\left(\bar{\theta} \bar{\sigma}_{\mu} \chi\right) \\
(\psi \chi)\left(\theta \sigma^{\mu} \bar{\eta}\right)=-\frac{1}{2}\left[\left(\psi \sigma^{\mu} \bar{\eta}\right)(\theta \chi)+2\left(\psi \sigma_{\nu} \bar{\eta}\right)\left(\theta \sigma^{\mu \nu} \chi\right)\right] \\
(\psi \chi)\left(\bar{\theta} \sigma^{\mu} \eta\right)=-\frac{1}{2}\left[(\psi \eta)\left(\bar{\theta} \sigma^{\mu} \chi\right)-2\left(\psi \sigma^{\mu \nu} \eta\right)\left(\bar{\theta} \bar{\sigma}_{\nu} \chi\right)\right] \\
\left(\psi \sigma^{\mu} \bar{\chi}\right)\left(\theta \sigma^{\nu} \bar{\eta}\right)=-\frac{1}{2}\left[\left(\psi \sigma^{\mu} \bar{\eta}\right)\left(\theta \sigma^{\nu} \bar{\chi}\right)-g^{\mu \nu}\left(\psi \sigma^{\rho} \bar{\eta}\right)\left(\theta \sigma_{\rho} \bar{\chi}\right)-i \epsilon^{\mu \nu \rho \sigma}\left(\psi \sigma_{\rho} \bar{\eta}\right)\left(\theta \sigma_{\sigma} \bar{\chi}\right)\right](\mathrm{C} .15) \\
\left(\psi \sigma^{\mu} \bar{\chi}\right)\left(\bar{\theta} \sigma^{\nu} \eta\right)=-\frac{1}{2}\left[g^{\mu \nu}(\psi \eta)(\bar{\theta} \bar{\chi})+2\left(\psi \sigma^{\mu \nu} \eta\right)(\bar{\theta} \bar{\chi})-2(\psi \eta)\left(\bar{\theta} \bar{\sigma}^{\mu \nu} \bar{\chi}\right)-4\left(\psi \sigma^{\nu \rho} \eta\right)\left(\bar{\theta} \bar{\sigma}_{\rho}^{\mu} \bar{\chi}\right)\right]
\end{gathered}
$$




\section{References}

[1] J. Wess and J. Bagger. Supersymmetry and supergravity. Princeton, USA: Univ. Pr. (1992) 259 p.

[2] D. Bailin and A. Love. Supersymmetric gauge field theory and string theory. Bristol, UK: IOP (1994) 322 p. (Graduate Student Series In Physics). 


\section{Appendix D}

\section{Computation of Supercompositeness}

\section{Diagram}

The supercompositeness diagram of figure 3.3 is more complicated than the other pseudoscalar contributions to pion decay that we have considered, because it involves a loop integral and a propagator that carries colour charge. Here, we compute its contribution in detail.

In supersymmetry, each fermion has a bosonic partner, called a superpartner, and vice versa. If supersymmetry were an unbroken symmetry, then the properties of a particle and its superpartner should be identical (except for spin). In particular, unbroken supersymmetry would imply that for each currently known particle, there exists a superpartner with the same mass and quantum numbers. However, such superpartners have not been experimentally observed. It follows that if supersymmetry exists in nature, it must be broken. We would expect the masses of the superpartners to be set by the scale of the physics that breaks supersymmetry, and thus $m_{g} \sim m_{\tilde{u}} \sim m_{\tilde{d}}$. To evaluate the supercompositeness amplitude more easily, we replace all of the superpartner masses by $m_{g}$. In addition, we ignore external momenta, so that all of the propagators in the loop have momentum $k$. The squark propagators are thus $\frac{1}{k^{2}-m_{g}^{2}}$, and the gluino propagator is $\frac{\not k+m_{g}}{k^{2}-m_{g}^{2}} \delta^{a b}$, where $1 \leq a, b \leq 8$ 
are colour indices.

We now examine the components of the amplitude that carry $S U(3)$ colour indices, in order to compute the colour factor of the diagram. Note that since the pion is a colour singlet, its two component particles must have opposite colour charge. The incoming particles are thus in the colour singlet $\frac{1}{\sqrt{3}}(r \bar{r}+b \bar{b}+g \bar{g})$. Combining this factor with the $\delta^{a b}$ from the gluino propagator, we get an overall colour factor of

$$
\frac{1}{\sqrt{3}}\left(r^{\dagger} T^{a} T^{a} r+b^{\dagger} T^{a} T^{a} b+g^{\dagger} T^{a} T^{a} g\right)
$$

where the $T^{a}$ are the $S U(3)$ matrices defined in equation 1.45 , and $r, b, g$ are unit vectors in the fundamental representation of $S U(3)$. The bracketed expression above is the trace of $T^{a} T^{a}$, and since $T^{a} T^{a}=\frac{4}{3} I$, the colour factor is

$$
\frac{1}{\sqrt{3}}\left(\frac{4}{3} \cdot 3\right)=\frac{4}{\sqrt{3}}
$$

We may account for all of the colour indices in the amplitude by including this factor in our result.

Now, neglecting complex phases and $\mathcal{O}(1)$ mixing coefficients between left-handed and right-handed squarks, there is a factor of $\sqrt{2} g_{s}$ at both quark-squark-gluino vertices. The portion of the amplitude involving the quarks and gluino is thus

$$
\begin{aligned}
\bar{u}_{R}\left(\frac{\not k+m_{g}}{k^{2}-m_{g}^{2}}\right) d_{L} & =\bar{u}_{R}\left(\frac{m_{g}}{k^{2}-m_{g}^{2}}\right) d_{L} \\
& =\bar{u}\left(\frac{m_{g}}{k^{2}-m_{g}^{2}}\right)\left(\frac{1-\gamma^{5}}{2}\right) d \\
& \rightarrow \frac{1}{2} \bar{u}\left(\frac{m_{g}}{k^{2}-m_{g}^{2}}\right) \gamma^{5} d
\end{aligned}
$$

where we have used $\bar{u}_{R} k d_{L}=0$, and extracted the part of the expression proportional to $\bar{u} \gamma^{5} d$ since the pion is a pseudoscalar particle (ignoring the sign). Multiplying by 
the leptonic portion of the amplitude, including the colour factor, and performing the loop integral, we have the result

$$
\begin{aligned}
\mathcal{M} & \sim \frac{4}{\sqrt{3}} \int \frac{d^{4} k}{(2 \pi)^{4}}\left(\sqrt{2} g_{s}\right)^{2} \frac{\bar{u} m_{g} \gamma^{5} d}{2\left(k^{2}-m_{g}^{2}\right)}\left(\frac{1}{k^{2}-m_{g}^{2}}\right)^{2} \frac{1}{M}\left[\bar{l}_{R} \nu_{L}\right] \\
& \sim \frac{4 g_{s}^{2} m_{g}}{\sqrt{3} M}\left[\bar{l}_{R} \nu_{L}\right]\left[\bar{u} \gamma^{5} d\right] \int \frac{\mathrm{d}^{4} k}{(2 \pi)^{4}}\left(\frac{1}{k^{2}-m_{g}^{2}}\right)^{3} .
\end{aligned}
$$

The loop integral is clearly convergent. It may be easily computed by using the formulae of appendix A and immediately setting $d=4$, yielding

$$
\begin{aligned}
\mathcal{M} & \sim \frac{4 g_{s}^{2} m_{\Delta}}{\sqrt{3} M}\left[\bar{l}_{R} \nu_{L}\right]\left[\bar{u} \gamma^{5} d\right]\left(\frac{1}{32 \pi^{2} m_{g}^{2}}\right) \\
& \sim \frac{\alpha_{s}}{2 \sqrt{3} \pi} \frac{1}{M m_{g}}\left[\bar{l}_{R} \nu_{L}\right]\left[\bar{u} \gamma^{5} d\right] .
\end{aligned}
$$

Finally, we use the identity

$$
\left\langle 0\left|\bar{u} \gamma^{5} d\right| \pi^{-}\right\rangle=i \sqrt{2} \tilde{f}_{\pi}
$$

and write the lepton chirality operator explicitly to obtain the final result

$$
\mathcal{M} \sim \frac{\alpha_{s}}{2 \sqrt{6} \pi} \frac{1}{M m_{g}}\left[\bar{l}\left(1-\gamma^{5}\right) \nu\right] \tilde{f}_{\pi}
$$

